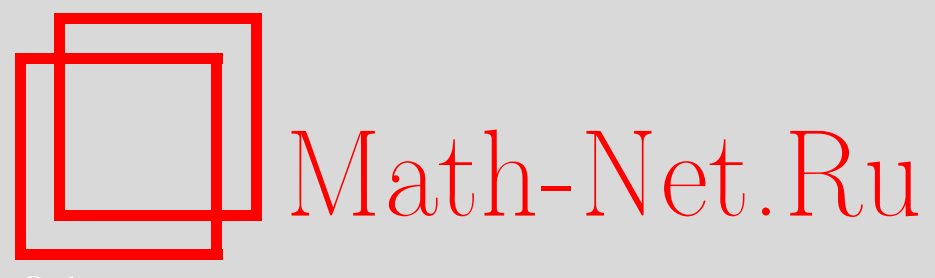

В. Л. Куракин, Линейная сложность полилинейных последовательностей, Дискрет. матем., 2001, том 13, выпуск $1,3-55$

DOI: https://doi.org/10.4213/dm274

Использование Общероссийского математического портала Math-Net.Ru подразумевает, что вы прочитали и согласны с пользовательским соглашением http: //www.mathnet.ru/rus/agreement

Параметры загрузки:

IP : 107.22 .136 .117

26 апреля 2023 г., $13: 27: 38$

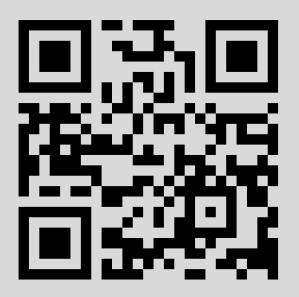


УДК 519.7

\title{
Линейная сложность полилинейных последовательностей
}

\author{
(C) 2001 г. $\quad$ В. Л. Куракин
}

\begin{abstract}
Вводится несколько определений линейной сложности (ранга) полилинейной рекуррентной последовательности над кольцом или модулем. Исследуется эквивалентность этих определений и свойства линейной сложности последовательностей над различными классами колец: полями, телами, коммутативными и коммутативными артиновыми кольцами, левыми областями Оре, областями Безу. Доказывается, что для последовательностей над коммутативными областями Безу, так же, как и для последовательностей над полем, все введенные определения линейной сложности эквивалентны.
\end{abstract}

\section{1. Введение}

Линейной сложностью или рангом линейной рекуррентной последовательности (сокращенно ЛРП или 1-ЛРП) над коммутативным кольцом традиционно называют степень ее минимального многочлена, то есть характеристического многочлена наименьшей степени. При определении линейной сложности полилинейной (или $k$-линейной) рекуррентной последовательности (сокращенно $k$-ЛРП) мы сталкиваемся с ситуацией, когда такое определение можно дать многими естественными способами. Получающиеся определения оказываются, в общем случае, не эквивалентными, и выбор того или иного из них диктуется потребностями прикладной задачи и классом колец, над которыми рассматриваются последовательности. В данной статье мы придерживаемся той точки зрения, что все вводимые ниже определения линейной сложности являются одинаково важными и заслуживают изучения.

Более подробно, мы вводим 18 определений линейной сложности последовательности $u$ и обозначаем соответствующие параметры через $r_{1}(u), \ldots, r_{18}(u)$ или просто $r_{1}, \ldots, r_{18}$. Эти определения возникли в результате анализа серии работ А. С. Кузьмина, А. В. Михалева, А. А. Нечаева и автора $[6,16,17]$, посвященных изучению линейных рекуррентных последовательностей над кольцами и модулями. Определения можно разбить на группы, характеризующие различные алгебраические и комбинаторные свойства ЛРП $u$. Для каждой $k$-последовательности можно рассмотреть ее модуль сдвигов, являющийся подмодулем в модуле всех последовательностей. Первая группа определений характеризует ранг модуля сдвигов ЛРП $u$. Как и при определении ранга модуля в общей алгебре, под рангом модуля сдвигов понимается либо наименьшее число образующих, либо наибольшее число линейно независимых элементов. Вторая группа определений характеризует возможность реализации последовательности $k$-линейным регистром сдвига. Несколько параметров можно условно 
назвать информационной линейной сложностью. Наконец, одно из определений линейной сложности является обобщением понятия степени минимального многочлена 1-ЛРП на случай $k$-ЛРП.

Понятие линейной сложности 1-ЛРП над полем использовалось в работах многих авторов (см. литературу в [16]). В основном в этих работах вычислялась или оценивалась линейная сложность различных классов последовательностей и их преобразований, а также исследовался алгоритм Берлекэмпа-Месси для практического нахождения линейной сложности заданной последовательности (см. $[14,15,20,24]$, а также $[8,9]$ и литературу в последних двух работах). Линейная сложность $k$ ЛРП над полем и над кольцом вычетов $Z_{m}$ рассматривалась в работах Сакаты (см., например, $[21,22,23])$ в связи с многомерным обобщением алгоритма БерлекэмпаМесси и построением $k$-линейных регистров сдвига. Линейная сложность $k$-ЛРП над коммутативными кольцами и модулями над ними рассматривалась в [6] также в связи с построением $k$-линейных регистров сдвига. Над некоммутативными кольцами $k$-линейные регистры сдвига рассматривались в [17].

В большинстве работ понятие линейной сложности играло вспомогательную роль как некоторый числовой параметр, характеризующий изучаемую последовательность или класс последовательностей. Как правило, при этом рассматривалась какаялибо одна линейная сложность последовательности, обычно степень минимального многочлена. Исключение составляет работа [6], в которой введены несколько определений линейной сложности и исследованы соотношения между ними (более подробно работа [6] комментируется далее в тексте). Цель данной статьи - исследовать соотношения между вводимыми ниже определениями линейной сложности и их эквивалентность над различными классами колец и модулей.

Имеющиеся примеры показывают, что большинство из вводимых определений линейной сложности не эквивалентны друг другу. В общем случае удалось доказать эквивалентность лишь двух из 18 определений. Возможно, есть и другие пары эквивалентных определений, но пока это не доказано и не опровергнуто примерами. При различных ограничениях на класс рассматриваемых колец и модулей некоторые из определений становятся эквивалентными. С этой точки зрения в статье рассмотрены классы коммутативных колец, артиновых колец, тел и полей, левых областей Оре, областей Безу. Отметим, что в конце работы приводятся две таблицы, в которых пгеречислены все полученные результаты и рассмотренные примеры.

Как было известно и ранее, для 1-ЛРП над полем все 18 вводимых ниже определений линейной сложности эквивалентны. Из имеющихся результатов [16] видно, что некоторые определения эквивалентны и для 1-ЛРП над кольцом целых чисел $\mathrm{Z}$. Одной из целей статьи было доказательство эквивалентности максимально возможного числа определений линейной сложности для 1-ЛРП над коммутативными областями главных идеалов. Как оказалось, эквивалентны все 18 определений, причем для 1-ЛРП над более широким классом колец - коммутативными областями Безу. Таким образом, теперь к утверждению работ $[10,11,17]$ о том, что теория семейств $k$-линейных рекуррент над полем наиболее полно переносится на рекурренты над квазифробениусовыми модулями, можно добавить еще одно: теория линейной сложности 1-ЛРП над полем наиболее полно сохраняется для 1-ЛРП над коммутативными областями Безу. В связи с этими двумя утверждениями отметим, что, как легко видеть, области целостности, являющиеся квазифробениусовыми кольцами, это в точности тела (поля в коммутативном случае). 


\section{2. Полилинейные рекуррентные последовательности}

Пусть $M$ - левый модуль над кольцом $R$ с единицей (в дальнейшем модуль означает левый модуль). Произвольное отображение $u: \mathbf{N}_{0} \rightarrow M$, где $\mathbf{N}_{0}=\{0,1,2, \ldots\}$, называется 1-последовательностью (или просто последовательностью) над $M$. Последовательность $u=(u(0), u(1), \ldots)$ называется линейной рекуррентной последовательностью (сокращенно 1-ЛРП или ЛРП), если существуют число $m \geqslant 1$ и элементы $c_{0}, c_{1}, \ldots, c_{m-1} \in R$ такие, что

$$
u(i+m)=c_{m-1} u(i+m-1)+\ldots+c_{1} u(i+1)+c_{0} u(i), \quad i \geqslant 0 .
$$

Многочлен

$$
F(x)=x^{m}-c_{m-1} x^{m-1}-\ldots-c_{1} x-c_{0} \in R[x]
$$

называется характеристическим многочленом ЛРП $u$, а вектор

$$
u[0, m-1]=(u(0), \ldots, u(m-1)) \in M^{m}
$$

называется ее начальным вектором. Характеристический многочлен наименьшей степени называется минимальным многочленом ЛРП $u$, а его степень обозначается rank $u$.

Если $k \geqslant 1$, то произвольное отображение $u: \mathbf{N}_{0}^{k} \rightarrow M$ называется $k$-последовательностью (или просто последовательностью) над $M$. Последовательность $u$ называется $k$-линейной рекуррентной последовательностью (сокращенно $k$-ЛРП или ЛРП) над модулем $M$, если существуют унитарные многочлены $F_{1}(x), \ldots, F_{k}(x)$ из $R[x]$ такие, что для любого $s \in\{1, \ldots, k\}$ и для любых $i_{1}, \ldots, i_{s-1}, i_{s+1}, \ldots, i_{k} \in \mathbf{N}_{0}$ 1-последовательность

$$
v(i)=u\left(i_{1}, \ldots, i_{s-1}, i, i_{s+1}, \ldots, i_{k}\right)
$$

есть 1-ЛРП над $M$ с характеристическим многочленом $F_{s}(x)$. Многочлены $F_{1}\left(x_{1}\right), \ldots, F_{k}\left(x_{k}\right)$ из кольца $R[x]=R\left[x_{1}, \ldots, x_{k}\right]$ многочленов от $k$ переменных называются элементарными характеристическими многочленами $k$-ЛРП $u$. Множество всех $k$-последовательностей над $M$ обозначается через $M^{\langle k\rangle}$, множество всех $k$-ЛРП над $M$ - через $\mathscr{L}_{R} M^{\langle k\rangle}$. Множество всех $k$-ЛРП над $M$ с элементарными характеристическими многочленами $F_{1}\left(x_{1}\right), \ldots, F_{k}\left(x_{k}\right)$ обозначается $L_{M}(\boldsymbol{F})=$ $L_{M}\left(F_{1}, \ldots, F_{k}\right)$.

Для $\boldsymbol{s}=\left(s_{1}, \ldots, s_{k}\right) \in \mathbf{N}_{0}^{k}$ обозначим $\boldsymbol{x}^{s}=x_{1}^{s_{1}} \ldots x_{k}^{s_{k}}$. Если $u \in M^{\langle k\rangle}$, то последовательность $v=\boldsymbol{x}^{\boldsymbol{s}} u \in M^{\langle k\rangle}$ со знаками $v(\boldsymbol{i})=u(i+\boldsymbol{s}), \boldsymbol{i} \in \mathbf{N}_{0}^{k}$, называется сдвигом последовательности $u$ на вектор $s$ (или на $s$ шагов влево при $k=1$ ). Произведение произвольной последовательности $u$ на многочлен

$$
F(x)=\sum_{\boldsymbol{s} \in \mathbf{N}_{\mathbf{0}}^{k}} c_{\boldsymbol{s}} \boldsymbol{x}^{\boldsymbol{s}} \in R[\boldsymbol{x}]
$$

определяется по линейности:

$$
v=F(\boldsymbol{x}) u=\sum_{\boldsymbol{s} \in \mathbf{N}_{0}^{k}} c_{\boldsymbol{s}} \boldsymbol{x}^{\boldsymbol{s}} u
$$


то есть

$$
v(i)=\sum_{s \in \mathbf{N}_{0}^{k}} c_{s} u(i+s), \quad i \in \mathbf{N}_{0}^{k} .
$$

Относительно этой операции абелева группа $\left(M^{\langle k\rangle},+\right)$ с естественным (покоординатным) сложением последовательностей превращается в левый $R[\boldsymbol{x}]$-модуль [16]. Будем говорить, что $F(\boldsymbol{x})$ аннулирует $u$, если $F(\boldsymbol{x}) u=0$. Левый идеал

$$
\operatorname{An}(u)=\{F(\boldsymbol{x}) \in R[\boldsymbol{x}]: F(\boldsymbol{x}) u=0\}
$$

кольца $R[\boldsymbol{x}]$ называется аннулятором последовательности $u$. Левый (правый, двусторонний) идеал $I$ кольца $R[\boldsymbol{x}]$ назовем унитарным, если он содержит многочлены $F_{1}\left(x_{1}\right), \ldots, F_{k}\left(x_{k}\right)$ такие, что $F_{s}(x)$ - унитарный многочлен от одной переменной, $s=1, \ldots, k$. Такие многочлены будем называть элементарными характеристическими многочленами идеала $I$. Согласно приведенным определениям, последовательность $u \in M^{\langle k\rangle}$ есть $k$-ЛРП тогда и только тогда, когда левый идеал $\operatorname{An}(u)$ унитарен, при этом $u \in L_{M}(\boldsymbol{F})$ тогда и только тогда, когда $F_{1}\left(x_{1}\right), \ldots, F_{k}\left(x_{k}\right) \in \operatorname{An}(u)$.

Подмодуль

$$
R[\boldsymbol{x}] u=\{F(\boldsymbol{x}) u: F(\boldsymbol{x}) \in R[\boldsymbol{x}]\}
$$

$R[\boldsymbol{x}]$-модуля $M^{\langle k\rangle}$ (то есть циклический подмодуль, порожденный элементом $u$ ) называется модулем сдвигов $k$-последовательности $u$, а $R[\boldsymbol{x}]$-модуль $R[\boldsymbol{x}] / \operatorname{An}(u)-$ eе модулем операторов. Если $\operatorname{An}(u)$ - двусторонний идеал в $R[\boldsymbol{x}]$, в частности, если $R$ коммутативно, то $R[\boldsymbol{x}] / \operatorname{An}(u)$ - кольцо, называемое кольцом операторов $k$ последовательности $u$. Для произвольного подмножества $\chi \subseteq R[\boldsymbol{x}]$ положим

$$
L_{M}(\chi)=\left\{u \in M^{\langle k\rangle}: \chi u=0\right\} .
$$

Тогда $L_{M}(\chi)$ является абелевой группой и $L_{M}(\chi)=L_{M}(R[\boldsymbol{x}] \chi)$, где $R[\boldsymbol{x}] \chi-$ левый идеал кольца $R[\boldsymbol{x}]$, порожденный множеством $\chi$. Если левый идеал $R[\boldsymbol{x}] \chi$ унитарен, то $L_{M}(\chi) \subseteq \mathscr{L}_{R} M^{\langle k\rangle}$ и абелева группа $L_{M}(\chi)$ называется ЛРП-семейством. Всякое ЛРП-семейство содержится в ЛРП-семействе вида $L_{M}(\boldsymbol{F})$. Если левый идеал $R[\boldsymbol{x}] \chi$ является также правым идеалом (в частности, если кольцо $R$ коммутативно), то $L_{M}(\chi)$ - левый $R[\boldsymbol{x}]$-подмодуль в $M^{\langle k\rangle}$.

На множестве $\mathbf{N}_{0}^{k}$ зададим отношения частичного (покоординатного) порядка $\leqslant$, полагая для $\boldsymbol{i}=\left(i_{1}, \ldots, i_{k}\right), \boldsymbol{j}=\left(j_{1}, \ldots, j_{k}\right) \in \mathbf{N}_{0}^{k}$

$$
i \leqslant j \Longleftrightarrow i_{1} \leqslant j_{1}, \ldots, i_{k} \leqslant j_{k},
$$

и полного (лексикографического) порядка $\preccurlyeq$ полагая $i \preccurlyeq j$ если $i=j$ или если в последовательности

$$
\left(j_{1}+\ldots+j_{k}\right)-\left(i_{1}+\ldots+i_{k}\right), \quad j_{1}-i_{1}, \ldots, j_{k}-i_{k}
$$

первое ненулевое число положительно. Конечное множество $\mathscr{F} \subset \mathbf{N}_{0}^{k}$ назовем диаграммой Ферре, если

$$
\forall i, j \in \mathbf{N}_{0}^{k}(i \in \mathscr{F}, j \leqslant i) \quad \Longrightarrow \quad(j \in \mathscr{F}) \text {. }
$$

Пусть $\mathbf{1}_{s}=(0, \ldots, 0,1,0, \ldots, 0) \in \mathbf{N}_{0}^{k}$, где единица находится на $s$-м месте, $s=$ $1, \ldots, k$, и $\boldsymbol{i}+\mathscr{F}=\{\boldsymbol{i}+\boldsymbol{j}: \boldsymbol{j} \in \mathscr{F}\}$. Назовем множество

$$
\Delta_{s} \mathscr{F}=\left(\mathbf{1}_{s}+\mathscr{F}\right) \backslash \mathscr{F}
$$


(внешней) границей диаграммы Ферре $\mathscr{F}$ в направлении $s$, а множество

$$
\Delta \mathscr{F}=\Delta_{1} \mathscr{F} \cup \ldots \cup \Delta_{k} \mathscr{F}
$$

(внешней) границей диаграммы Ферре $\mathscr{F}$. Обозначим через $\mathbf{0}$ и 1 векторы из $\mathbf{N}_{0}^{k}$, все координаты которых равны 0 и 1 соответственно.

Элементы произвольного конечного подмножества $\Omega=\left\{\boldsymbol{i}_{1}, \ldots, \boldsymbol{i}_{m}\right\} \subset \mathbf{N}_{0}^{k}$ можно расположить в виде цепи $i_{1} \preccurlyeq \ldots \preccurlyeq i_{m}, m=|\Omega|$ (в данном случае порядок элементов множества $\Omega$ несущественен, лишь бы он был фиксированным). Вектор

$$
u[\Omega]=\left(u\left(i_{1}\right), \ldots, u\left(i_{m}\right)\right) \in M^{m}
$$

будем называть диаграммой (или вектором) значений $k$-последовательности $u$ на множестве $\Omega$. Если $u-k$-ЛРП с элементарными характеристическими многочленами $F_{1}\left(x_{1}\right), \ldots, F_{k}\left(x_{k}\right)$ степеней $m_{1}, \ldots, m_{k}$ соответственно, то множество

$$
\Pi=\Pi(\boldsymbol{m})=\left\{0,1, \ldots, m_{1}-1\right\} \times \ldots \times\left\{0,1, \ldots, m_{k}-1\right\}
$$

назовем начальным параллелепипедом $k$-ЛРП $u$, а $u[\Pi]-$ диаграммой начальных значений (или начальным вектором) $k$-ЛРП $u$. Так же, как и элементарные характеристические многочлены, начальный параллелепипед $k$-ЛРП $u$ определяется не однозначно.

Пусть $M^{\Omega}-$ множество всех отображений $\delta: \Omega \rightarrow M$. Вектор

$$
\delta[\Omega]=\left(\delta\left(i_{1}\right), \ldots, \delta\left(i_{m}\right)\right) \in M^{m}
$$

назовем диаграммой (или вектором) значений отображения $\delta$ на множестве $\Omega$. В дальнейшем мы будем отождествлять отображение $\delta \in M^{\Omega}$ с его вектором значений $\delta[\Omega] \in M^{m}$ и считать, что $M^{\Omega}=M^{m}$.

\section{3. Определения линейной сложности}

Пусть $u-k$-ЛРП над модулем ${ }_{R} M, R[\boldsymbol{x}] u$ - ее модуль сдвигов, рассматриваемый ниже как левый $R$-модуль, $\Omega$ и $\mathscr{F}-$ конечное подмножество и диаграмма Ферре в $\mathrm{N}_{0}^{k},{ }_{R}(S)$ - левый $R$-модуль, порожденный множеством $S$. Введем следующие обозначения:

$$
\begin{aligned}
& r_{1}=\min \{t \geqslant 0: R[\boldsymbol{x}] u \text { порождается } t \text { элементами }\}, \\
& r_{2}=\min \left\{|\Omega|: R[\boldsymbol{x}] u={ }_{R}\left(\boldsymbol{x}^{i} u, i \in \Omega\right)\right\}, \\
& r_{3}=\min \left\{|\mathscr{F}|: R[\boldsymbol{x}] u={ }_{R}\left(\boldsymbol{x}^{i} u, i \in \mathscr{F}\right)\right\}, \\
& r_{4}=\max \{t \geqslant 0: R[\boldsymbol{x}] u \text { содержит } t \text { линейно независимых элементов }\}, \\
& r_{5}=\max \left\{|\Omega|:\left(\boldsymbol{x}^{i} u, i \in \Omega\right) \text { линейно независимы }\right\}, \\
& r_{6}=\max \left\{|\mathscr{F}|:\left(\boldsymbol{x}^{i} u, i \in \mathscr{F}\right) \text { линейно независимы }\right\}, \\
& r_{7}=\min \left\{t \geqslant 0: \exists \text { мономорфизм } R \text {-модулей } R[\boldsymbol{x}] u \rightarrow M^{t}\right\}, \\
& r_{8}=\min \left\{|\Omega|: R[\boldsymbol{x}] u \rightarrow M^{\Omega}, v \rightarrow v[\Omega]-\text { мономорфизм }\right\}, \\
& r_{9}=\min \left\{|\mathscr{F}|: R[\boldsymbol{x}] u \rightarrow M^{\mathscr{F}}, v \rightarrow v[\mathscr{F}]-\text { мономорфизм }\right\} .
\end{aligned}
$$


Для краткости мы пишем $r_{i}$ вместо $r_{i}(u)$. Введенные параметры характеризуют (с алгебраической и комбинаторной точек зрения) ранг модуля сдвигов $k$-ЛРП $u$. Для $k$-ЛРП над полем параметры $r_{1}, \ldots, r_{6}$ исследовались в [6]. Отображения в определениях $r_{7}, \ldots, r_{9}$ являются мономорфизмами $R$-модулей. Множество $\Omega$ и диаграмму $\mathscr{F}$ из определений $r_{8}, r_{9}$ можно назвать определяющими множеством и диаграммой Ферре $k$-ЛРП $u$. Очевидно,

$$
r_{1} \leqslant r_{2} \leqslant r_{3}, \quad r_{4} \geqslant r_{5} \geqslant r_{6}, \quad r_{7} \leqslant r_{8} \leqslant r_{9} .
$$

Здесь и далее запись $r_{i} \leqslant r_{j}$ используется для сокращенной записи того, что $r_{i}(u) \leqslant$ $r_{j}(u)$ для всех $u$ из рассматриваемого класса $k$-ЛРП (в данном случае для всех $k$ ЛРП над любым модулем $\left.{ }_{R} M\right)$. Для произвольного левого $R$-модуля $N$ положим

$$
\begin{aligned}
& r_{1}(N)=\min \{t \geqslant 0: N \text { порождается } t \text { элементами }\}, \\
& r_{4}(N)=\max \{t \geqslant 0: N \text { содержит } t \text { линейно независимых элементов }\} .
\end{aligned}
$$

Тогда

$$
r_{1}(u)=r_{1}(R[\boldsymbol{x}] u), \quad r_{4}(u)=r_{4}(R[\boldsymbol{x}] u) .
$$

Справедливы равенства

$$
\begin{aligned}
& r_{1}=\min \left\{t \geqslant 0: \exists \text { эпиморфизм } R^{t} \rightarrow R[\boldsymbol{x}] u\right\}, \\
& r_{2}=\min \left\{|\Omega|: R^{\Omega} \rightarrow R[\boldsymbol{x}] u, \delta[\Omega] \rightarrow \sum_{i \in \Omega} \delta(\boldsymbol{i}) \boldsymbol{x}^{\boldsymbol{i}} u-\text { эпиморфизм }\right\}, \\
& r_{3}=\min \left\{|\mathscr{F}|: R^{\mathscr{F}} \rightarrow R[\boldsymbol{x}] u, \delta[\mathscr{F}] \rightarrow \sum_{\boldsymbol{i} \in \mathscr{F}} \delta(\boldsymbol{i}) \boldsymbol{x}^{i} u-\text { эпиморфизм }\right\},
\end{aligned}
$$

поэтому определения $r_{1} \ldots, r_{3}$ и $r_{7}, \ldots, r_{9}$ можно считать двойственными друг другу.

Множество $\Phi=\left\{H_{\boldsymbol{r}}(\boldsymbol{x}): \boldsymbol{r} \in \Delta \mathscr{F}\right\}$ многочленов из $R[\boldsymbol{x}]$ вида

$$
H_{r}(x)=x^{r}-\sum_{i \in \mathscr{F}} h_{r i} x^{i}
$$

назовем полной системой $\mathscr{F}$-унитарных многочленов. Обозначим через $r_{10}=r_{10}(u)$ наименьшую мощность диаграммы $\Phi$ ерре $\mathscr{F}$, для которой существует полная система $\mathscr{F}$-унитарных многочленов $\Phi \subset \operatorname{An}(u)$. Зная такую систему многочленов $\Phi$ и значения $u[\mathscr{F}]$, можно найти всю ЛРП $u$, пользуясь рекуррентными соотношениями, задаваемыми многочленами из $\Phi$. Если $k=1$, то $r_{10}(u)$ есть не что иное, как степень минимального многочлена ЛРП $u$, то есть при $k=1$

$$
r_{10}(u)=\operatorname{rank} u \text {. }
$$

Поэтому параметр $r_{10}(u)$ является обобщением понятия степени минимального многочлена 1 -ЛРП на случай $k$-ЛРП при $k \geqslant 1$. Множества $\mathscr{F}$-унитарных многочленов и параметр $r_{10}$ рассматривались ранее в $[21,22,23,16]$.

Напомним [17], что пара $\langle\chi, \mathscr{F}\rangle$, где $\chi \subseteq R[\boldsymbol{x}]$, называется $k$-линейным регистром сдвига ( $k$-ЛРС), если отображение

$$
\sigma: L_{M}(\chi) \rightarrow M^{\mathscr{F}}, \quad v \rightarrow v[\mathscr{F}]
$$


биективно (и следовательно, является изоморфизмом абелевых групп, а если $R$ коммутативно, то и $R$-модулей). Если $u \in L_{M}(\chi)$, то говорят, что $u$ является выходной последовательностью или выходом $k$-ЛРС $\langle\chi, \mathscr{F}\rangle$. Следуя [17], положим

$$
r_{11}=\min \{|\mathscr{F}|: \exists k \text {-ЛРC }\langle\chi, \mathscr{F}\rangle \text { с выходом } u\} .
$$

В [6] введены два параметра, сходные с $r_{11}$. Пусть $E=\operatorname{End}\left(M^{\mathscr{F}}\right)-$ кольцо эндоморфизмов $R$-модуля $M^{\mathscr{F}}=M^{|\mathscr{F}|}$. Действие эндоморфизма $\varphi: M^{\mathscr{F}} \rightarrow M^{\mathscr{F}}$ на элемент $\delta[\mathscr{F}] \in M^{\mathscr{F}}$ можно записать следующим образом:

$$
\varphi(\delta[\mathscr{F}])=\left(\varphi_{\boldsymbol{i}}(\delta[\mathscr{F}]), \quad i \in \mathscr{F}\right) \in M^{\mathscr{F}},
$$

где $\varphi_{i}: M^{\mathscr{F}} \rightarrow M, i \in \mathscr{F},-$ компоненты эндоморфизма $\varphi$ (мы пишем действие эндоморфизма $\varphi$ слева, хотя $M^{\mathscr{F}}$ является правым $E$-модулем и $(R, E)$-бимодулем). Эндоморфизм $\varphi \in E$ будем называть скалярным (слева), если все его компоненты - скалярные (слева) гомоморфизмы, то есть

$$
\varphi_{\boldsymbol{i}}(\delta[\mathscr{F}])=\sum_{\boldsymbol{j} \in \mathscr{F}} c_{\boldsymbol{i}} \delta(\boldsymbol{j})
$$

для некоторых $c_{i j} \in R$. Множество всех скалярных эндоморфизмов обозначается $\widehat{E}=\widehat{\operatorname{End}}\left(M^{\mathscr{F}}\right)$. Для $\varphi_{1}, \ldots, \varphi_{k} \in E, \boldsymbol{i}=\left(i_{1}, \ldots, i_{k}\right) \in \mathbf{N}_{0}^{k}$, обозначим

$$
\varphi^{i}=\varphi_{1}^{i_{1}} \ldots \varphi_{k}^{i_{k}}
$$

Положим

$$
\begin{gathered}
r_{12}=\min \left\{|\mathscr{F}|: \exists \text { попарно перестановочные } \varphi_{1}, \ldots, \varphi_{k} \in E\right. \\
\text { такие, что } \left.u[i+\mathscr{F}]=\varphi^{i}(u[\mathscr{F}]), i \in \mathbf{N}_{0}^{k}\right\}, \\
r_{13}=\min \left\{|\mathscr{F}|: \exists \text { попарно перестановочные } \varphi_{1}, \ldots, \varphi_{k} \in \widehat{E}\right. \\
\text { такие, что } \left.u[i+\mathscr{F}]=\varphi^{i}(u[\mathscr{F}]), i \in \mathbf{N}_{0}^{k}\right\} .
\end{gathered}
$$

Для эндоморфизмов $\varphi_{1}, \ldots, \varphi_{k}$, удовлетворяющих определению $r_{12}$ или $r_{13}$, выполняется также следующее условие: если диаграмма значений $\delta[\mathscr{F}] \in M^{\mathscr{F}}$ принадлежит множеству

$$
\left\{u[i+\mathscr{F}]: i \in \mathbf{N}_{0}^{k}\right\}, \quad s \in\{1, \ldots, k\}, \quad \lambda[\mathscr{F}]=\varphi_{s}(\delta[\mathscr{F}]),
$$

To

$$
\boldsymbol{j} \in \mathscr{F}, \quad \boldsymbol{j}+\mathbf{1}_{s} \in \mathscr{F} \quad \Longrightarrow \quad \lambda(\boldsymbol{j})=\delta\left(\boldsymbol{j}+\mathbf{1}_{s}\right)
$$

Действительно, если $\delta[\mathscr{F}]=u[\boldsymbol{i}+\mathscr{F}]$, то

$$
\lambda[\mathscr{F}]=\varphi_{s}(u[\boldsymbol{i}+\mathscr{F}])=u\left[\boldsymbol{i}+\mathbf{1}_{s}+\mathscr{F}\right]
$$

и следовательно,

$$
\lambda(\boldsymbol{j})=u\left(i+\mathbf{1}_{s}+\boldsymbol{j}\right)=\delta\left(\boldsymbol{j}+\mathbf{1}_{s}\right)
$$

при условии, что $\boldsymbol{j}+\mathbf{1}_{s} \in \mathscr{F}$. Естественно потребовать, чтобы эндоморфизмы $\varphi_{1}, \ldots, \varphi_{k}$ удовлетворяли этому условию для всех $\delta[\mathscr{F}] \in M^{\mathscr{F}}$. Следуя [6], попарно перестановочные эндоморфизмы $\varphi_{1}, \ldots, \varphi_{k} \in E$, удовлетворяющие следующему условию: для всех $\delta[\mathscr{F}] \in M^{\mathscr{F}}, s \in\{1, \ldots, k\}$ и $\boldsymbol{j} \in \mathscr{F}$

$$
\left(\boldsymbol{j}+\mathbf{1}_{s} \in \mathscr{F}, \lambda[\mathscr{F}]=\varphi_{s}(\delta[\mathscr{F}]) \quad \Longrightarrow \quad \lambda(\boldsymbol{j})=\delta\left(\boldsymbol{j}+\mathbf{1}_{s}\right)\right),
$$


назовем $\mathscr{F}$-ЛРС системой. Если эндоморфизм $\varphi_{s}$ записать в виде $\varphi_{s}=\left(\varphi_{s j}, j \in \mathscr{F}\right)$, где $\varphi_{s j}: M^{\mathscr{F}} \rightarrow M-$ компоненты $\varphi_{s}$, то из равенства $\lambda[\mathscr{F}]=\varphi_{s}(\delta[\mathscr{F}])$ следует, что $\lambda(j)=\varphi_{s j}(\delta[\mathscr{F}]), j \in \mathscr{F}$, и условие (5) запишется в следующем виде: для всех $\delta[\mathscr{F}] \in M^{\mathscr{F}}, s \in\{1, \ldots, k\}$ и $j \in \mathscr{F}$

$$
\left(\boldsymbol{j}+\mathbf{1}_{s} \in \mathscr{F} \quad \Longrightarrow \quad \varphi_{s j}(\delta[\mathscr{F}])=\delta\left(j+\mathbf{1}_{s}\right)\right)
$$

Положим

$$
\begin{aligned}
r_{14}= & \min \left\{|\mathscr{F}|: \exists \mathscr{F} \text {-ЛРС система } \varphi_{1}, \ldots, \varphi_{k} \in E\right. \text { такая, } \\
& \text { что } \left.u[i+\mathscr{F}]=\varphi^{i}(u[\mathscr{F}]), i \in \mathbf{N}_{0}^{k}\right\}, \\
r_{15}= & \min \left\{|\mathscr{F}|: \exists \mathscr{F} \text {-ЛРС система } \varphi_{1}, \ldots, \varphi_{k} \in \widehat{E}\right. \text { такая, } \\
& \text { что } \left.u[i+\mathscr{F}]=\varphi^{i}(u[\mathscr{F}]), i \in \mathbf{N}_{0}^{k}\right\} .
\end{aligned}
$$

В [6] параметры $r_{12}$ и $r_{13}$ названы $M$-рангом и $R$-рангом соответственно, поэтому $r_{14}$ и $r_{15}$ можно назвать $M$-ЛРС-рангом и $R$-ЛРС-рангом. Очевидно,

$$
r_{12} \leqslant r_{13}, \quad r_{14} \leqslant r_{15}, \quad r_{12} \leqslant r_{14}, \quad r_{13} \leqslant r_{15}
$$

В условиях определений $r_{10}, \ldots, r_{15}$ диаграмму Ферре $\mathscr{F}$ можно назвать определяющей диаграммой ЛРП $u$, поскольку, зная $u[\mathscr{F}]$ (и систему многочленов $\Phi$ или эндоморфизмов $\varphi$ ), можно найти все знаки последовательности $u$. Параметры $r_{11}, \ldots, r_{15}$ характеризуют сложность выработки $k$-ЛРП $u$ линейным регистром сдвига.

Положим

$$
\begin{aligned}
& r_{16}=\min \{t \geqslant 0: R[\boldsymbol{x}] u \text { содержится в } t \text {-порожденном } R \text {-модуле }\}, \\
& r_{17}=\min \left\{r_{1}\left(L_{M}(I)\right): u \in L_{M}(I), I-\text { унитарный правый идеал в } R[\boldsymbol{x}]\right\} .
\end{aligned}
$$

Так как $I$ в определении $r_{17}$ - правый идеал, то $L_{M}(I)-$ левый $R$-модуль и обозначение $r_{1}\left(L_{M}(I)\right)$ корректно. При этом условии включение $u \in L_{M}(I)$ равносильно включению $R[\boldsymbol{x}] u \subseteq L_{M}(I)$. Параметры $r_{16}$ и $r_{17}$ характеризуют ранг модуля сдвигов ЛРП $и$. Очевидно,

$$
r_{1} \geqslant r_{16}
$$

Если кольцо $R$ конечно, то определим

$$
r_{18}=r_{18}(u)=\log _{|R|}|R[\boldsymbol{x}] u|
$$

Этот параметр можно назвать информационной линейной сложностью. Такой же термин, но уже в другом смысле, отчасти применим к $r_{7}, \ldots, r_{9}$, поскольку эти параметры показывают, по скольким знакам последовательность $v \in R[\boldsymbol{x}] u$ определяется однозначно. При использовании параметра $r_{18}$ подразумевается, что кольцо $R$ конечно, даже если это не указано явно.

Заметим, что для $k$-ЛРП $u \in \mathscr{L}_{R} M^{\langle k\rangle}$ параметры

$$
r_{i}(u), \quad i=1,2,3,5,6,10,16
$$

всегда конечны. С другой стороны, некоторые параметры, например, $r_{4}$ и $r_{11}$, могут обращаться в $\infty$. 
Пусть $R \subseteq Q-$ расширение кольца $R$. Тогда всякую последовательность над $R$ можно рассматривать как последовательность над $Q$. Будем обозначать через $r_{i}=r_{i}(u)=r_{i}^{R}(u)$ и $r_{i}^{Q}=r_{i}^{Q}(u), i=1, \ldots, 18$, линейную сложность ЛРП $u$, рассматриваемой как последовательность над $R$ и над $Q$ соответственно. Если $u$ - последовательность над $R$-модулем $M$, то обозначим через $[1 \otimes u]$ последовательность над $Q$-модулем $Q \stackrel{R}{\otimes} M$ со знаками

$$
[1 \otimes u](i)=1 \otimes u(i) \in Q \stackrel{R}{\otimes} M, \quad i \in \mathbf{N}_{0}^{k}
$$

(в дальнейшем будем писать $\otimes$ вместо $\stackrel{R}{\otimes}$ ).

Линейную сложность ЛРП $[1 \otimes u]$ над $Q$-модулем $Q \otimes M$ будем обозначать $r_{i}^{Q}=$ $r_{i}^{Q}(1 \otimes u)$. Если $M=R$, то $Q$-модули $Q$ и $Q \otimes M$ канонически изоморфны, и мы отождествляем последовательности $u$ и $[1 \otimes u]$. Параметры $r_{i}^{Q}(u)$ и $r_{i}^{Q}(1 \otimes u)$ будем называть линейной сложностью ЛРП $u$ над расширением кольца $R$ и модуля $M$ соответственно. Отметим, что последовательность $[1 \otimes u] \in(Q \otimes M)^{\langle k\rangle}$ нельзя путать с элементом $1 \otimes u \in Q \otimes M^{\langle k\rangle}$, который не является последовательностью (в случае, когда $R$ - поле, этот вопрос подробно рассмотрен в [19]). Чтобы подчеркнуть различие, мы используем квадратные скобки, которые, однако, опускаем в записи $r_{i}^{Q}(1 \otimes u)$.

Существуют и другие естественные способы определения линейной сложности, которые не исследуются в этой работе. Пусть $u-k$-ЛРП над коммутативным кольцом $R$ и $\Pi-$ некоторый ее начальный параллелепипед, $|\Pi|=m$. Из векторов столбцов $(u[i+\Pi], i \in \Pi)$ составим матрицу $U$ размера $m \times m$. Если $k=1$, то $U-$ ганкелева матрица ЛРП $u$. Положим

$$
r_{19}(u)=\operatorname{rank} U, \quad r_{20}(u)=\operatorname{rk} U
$$

где $\operatorname{rank} U-$ ранг матрицы $U$, то есть наибольший порядок ненулевых миноров, а $\operatorname{rk} U-$ аннуляторный ранг, то есть наибольшее $t \geqslant 0$ такое, что $\operatorname{An}_{R}\left(I_{U}(t)\right)=0$, где $I_{U}(t)$ - идеал кольца $R$, порожденный всеми минорами порядка $t$ матрицы $U$ (см. $[18,3])$.

Отметим также, что всякая $k$-ЛРП над коммутативным артиновым кольцом $R$ может быть представлена в виде суммы так называемых биномиальных последовательностей над некоторым расширением $Q$ исходного кольца (см. [7]). Если $u-k$ ЛРП над $R$-модулем $M$, то последнее утверждение применимо к последовательности $[1 \otimes u]$. Число биномиальных последовательностей в указанном представлении, подсчитанное надлежащим образом, называется биномиальной линейной сложностью ЛРП $u$. Обозначим этот параметр через $r_{21}(u)$. В большинстве работ по изучению линейной сложности 1-ЛРП над полем фактически находится или оценивается параметр $r_{21}$ и затем используется равенство (которое часто даже не упоминается явно) $r_{21}=r_{10}$, где $r_{10}(u)=\operatorname{rank} u-$ степень минимального многочлена 1 -ЛРП $u$. Например, так делалось в работах $[9,14,15,20,24]$. Для $k$-ЛРП аналогичный прием использовался в $[16, \S 24]$. Параметр $r_{21}$ имеет большое прикладное значение и заслуживает отдельного исследования.

Если $E=\operatorname{End}(M)-$ кольцо эндоморфизмов $R$-модуля $M$, то $M$ можно рассматривать как правый $E$-модуль. Линейную сложность ЛРП $u \in \mathscr{L}_{R} M^{\langle k\rangle}$, рассматриваемую как последовательность над правым модулем $M_{E}$, обозначим $r_{i}^{E}(u)$, $i=1, \ldots, 21$, и назовем эндолинейной сложностью (или эндорангом). Если вместо 
$E$ взять кольцо $\mathbf{E}=\operatorname{End}(M,+)$ эндоморфизмов абелевой группы $(M,+)$ (то есть $Z$ модуля $M)$, то соответствующую линейную сложность обозначим $r_{i}^{\mathbf{E}}(u)$. Более того, если $M$ является модулем (левым или правым) над некоторым кольцом $C$, то через $r_{i}^{C}(u)$ обозначим линейную сложность последовательности $u$, рассматриваемой как последовательность над $C$-модулем $M$. В качестве $C$ можно взять, например, центр кольца $R$. Указанные параметры можно назвать линейной сложностью над различными кольцами коэффициентов.

\section{4. Переход от модуля сдвигов к модулю операторов и от последовательностей к векторам}

Следующее предложение позволяет при оценке линейной сложности $k$-ЛРП рассматривать ее модуль операторов (кольцо операторов, если $R$ коммутативно) $R[\boldsymbol{x}] / \operatorname{An}(u)$ вместо модуля сдвигов $R[\boldsymbol{x}] u$. Это, в частности, дает возможность использовать результаты теории базисов Гребнера в кольцах многочленов для оценки линейной сложности (см. $[21,22,23]$ в случае $k$-ЛРП над полями и кольцами вычетов).

Предложение 1. Модуль сдвигов $k$-последователъности и изоморфен ее модулю операторов. Изоморфизм $\tau$ этих $R$-модулей $u R[\boldsymbol{x}]$-модулей задается соотношениeM

$$
\tau: R[\boldsymbol{x}] / \operatorname{An}(u) \rightarrow R[\boldsymbol{x}] u, \quad F(\boldsymbol{x})+\operatorname{An}(u) \rightarrow F(\boldsymbol{x}) u .
$$

Доказательство. Пусть $\varphi: R[\boldsymbol{x}] \rightarrow R[\boldsymbol{x}] u$ - отображение, задаваемое правилом $F(\boldsymbol{x}) \rightarrow F(\boldsymbol{x}) u$. Очевидно, $\varphi$ является эпиморфизмом левых $R$-модулей и $R[\boldsymbol{x}]-$ модулей с ядром $\operatorname{An}(u)$, и предложение следует из теоремы об эпиморфизме.

Отметим, что предложение 1 справедливо для произвольного циклического подмодуля: если ${ }_{R} M-$ модуль над кольцом $R$ и $m \in M$, то $R m \cong R / \operatorname{An}_{R}(m)$.

Следствие 1. Пусть $R[x] / \operatorname{An}(u)$ - модуль операторов $k$-ЛРП $u \in M^{\langle k\rangle}$, рассматриваемый как $R$-модулъ. Тогда справедливъ равенства

$$
\begin{aligned}
& r_{1}=\min \{t \geqslant 0: R[\boldsymbol{x}] / \operatorname{An}(u) \text { порождаетсл }(\text { над } R) t \text { элементами }\}, \\
& r_{2}=\min \left\{|\Omega|: R[\boldsymbol{x}] / \operatorname{An}(u)={ }_{R}\left(\boldsymbol{x}^{i}+\operatorname{An}(u), i \in \Omega\right)\right\}, \\
& r_{3}=\min \left\{|\mathscr{F}|: R[\boldsymbol{x}] / \operatorname{An}(u)={ }_{R}\left(\boldsymbol{x}^{i}+\operatorname{An}(u), i \in \mathscr{F}\right)\right\}, \\
& r_{4}=\max \{t \geqslant 0: R[\boldsymbol{x}] / \operatorname{An}(u) \text { содержит } t \text { линейно независимых элементов }\}, \\
& r_{5}=\max \left\{|\Omega|:\left(x^{i}+\operatorname{An}(u), i \in \Omega\right) \text { линейно независимы }\right\}, \\
& r_{6}=\max \left\{|\mathscr{F}|:\left(x^{i}+\operatorname{An}(u), i \in \mathscr{F}\right) \text { линейно независимы }\right\} .
\end{aligned}
$$

Модуль сдвигов $R[\boldsymbol{x}] u$, даже если $R$ и $M$ конечны, состоит из бесконечных последовательностей. На самом деле, в случае коммутативного кольца $R$, при нахождении линейной сложности $r_{1}, \ldots, r_{6}$ для проверки того, что $k$-ЛРП $v_{1}, \ldots, v_{n} \in R[\boldsymbol{x}] u$ порождают $R$-модуль $R[\boldsymbol{x}] u$ или являются линейно независимыми над $R$, можно ограничиться начальными параллелепипедами этих последовательностей.

Предложение 2. Пусть $R$ коммутативно, $u \in \mathscr{L}_{R} M^{\langle k\rangle}, \Pi=\Pi(\boldsymbol{m})$ - некоторъй начальный параллелепипед $k$-ЛІРП $u$. 
(a) Последовательности $v_{1}, \ldots, v_{n} \in R[\boldsymbol{x}] u$ порождают $R$-модуль $R[\boldsymbol{x}] u$ тогда $u$ только тогда, когда векторы $v_{1}[\Pi], \ldots, v_{n}[\Pi] \in M^{\Pi}$ порождают $R$-модуль ${ }_{R}\left(\boldsymbol{x}^{i} u[\Pi], i \in \Pi\right) \subseteq M^{\Pi}$.

(b) Для произволъных последовательностей $v_{1}, \ldots, v_{n} \in R[\boldsymbol{x}]$ справедливы импликации

$$
c_{1} v_{1}+\ldots+c_{n} v_{n}=0 \Longleftrightarrow c_{1} v_{1}[\Pi]+\ldots+c_{n} v_{n}[\Pi]=0
$$

$B$ частности, последователъности $v_{1}, \ldots, v_{n} \in R[\boldsymbol{x}]$ линейно независимы над $R$ тогда и толъко тогда, когда векторы $v_{1}[\Pi], \ldots, v_{n}[\Pi] \in M^{\Pi}$ линейно независимы над $R$.

Доказательство. Пусть $\boldsymbol{m}=\left(m_{1}, \ldots, m_{k}\right)$ и $F_{1}\left(x_{1}\right), \ldots, F_{k}\left(x_{k}\right)$ - элементарные характеристические многочлены ЛРП $u$ степеней $m_{1}, \ldots, m_{k}$ соответственно.

Необходимость в утверждении (а) очевидна. Докажем достаточность. Пусть $w \in R[\boldsymbol{x}] u$. Тогда $w=A(\boldsymbol{x}) u, A(\boldsymbol{x}) \in R[\boldsymbol{x}]$. Разделив $A(x)$ на многочлены $F_{1}\left(x_{1}\right), \ldots, F_{k}\left(x_{k}\right)$, получим представление

$$
A(\boldsymbol{x})=Q_{1}(\boldsymbol{x}) F_{1}\left(x_{1}\right)+\ldots+Q_{k}(\boldsymbol{x}) F_{k}\left(x_{k}\right)+a(\boldsymbol{x})
$$

где степень многочлена $a(\boldsymbol{x})$ по переменной $x_{s}$ меньше $m_{s}, s=1, \ldots, k$. При этом $w=a(\boldsymbol{x}) u$ и, следовательно, $w \in{ }_{R}\left(\boldsymbol{x}^{i} u, i \in \Pi\right)$. В силу условия

$$
w[\Pi]=c_{1} v_{1}[\Pi]+\ldots+c_{n} v_{n}[\Pi]
$$

для некоторых $c_{1}, \ldots, c_{n} \in R$. Тогда $w$ и $c_{1} v_{1}+\ldots+c_{n} v_{n}-$ две $k$-ЛРП с одинаковыми диаграммами значений на П и с элементарными характеристическими многочленами $F_{1}\left(x_{1}\right), \ldots, F_{k}\left(x_{k}\right)$. Следовательно, $w=c_{1} v_{1}+\ldots+c_{n} v_{n}$, и последовательности $v_{1}, \ldots, v_{n}$ порождают $R$-модуль $R[\boldsymbol{x}] u$. (Коммутативность кольца $R$ используется в утверждениях о том, что $w, v_{s}, c_{1} v_{1}+\ldots+c_{n} v_{n}-$ ЛРП с элементарными характеристическими многочленами $F_{1}\left(x_{1}\right), \ldots, F_{k}\left(x_{k}\right)$.)

Необходимость в утверждении (b) очевидна. Докажем достаточность. Предположим, что

$$
c_{1} v_{1}[\Pi]+\ldots+c_{n} v_{n}[\Pi]=0
$$

Тогда $c_{1} v_{1}+\ldots+c_{n} v_{n}$ и $0-$ две $k$-ЛРП с одинаковыми диаграммами значений на П и с элементарными характеристическими многочленами $F_{1}\left(x_{1}\right), \ldots, F_{k}\left(x_{k}\right)$. Следовательно, $c_{1} v_{1}+\ldots+c_{n} v_{n}=0$, откуда $c_{1}=\ldots=c_{n}=0$.

Отметим, что начальный параллелепипед П в предложении 2(а) можно заменить на определяющую диаграмму Ферре $\mathscr{F}$ ЛРП $u$ из определения $r_{10}$, а в предложении $2(\mathrm{~b})$ на множество $\Omega$ из определения $r_{8}$ или диаграмму Ферре $\mathscr{F}$ из определений $r_{9}, \ldots, r_{15}$. Доказательства в этих случаях аналогичны приведенному выше.

\section{5. Несколько примеров}

Сравнение различных определений линейной сложности мы начнем с нескольких примеров, показывающих, что эти определения могут быть не эквивалентными. Говорят, что кольцо $R$ удовлетворяет свойству ИБЧ (инвариантности базисного числа), если из $R^{m} \cong R^{n}$ следует, что $m=n$. Всякое кольцо, обладающее гомоморфизмом в 
тело, в частности, всякое коммутативное кольцо, обладает свойством ИБЧ (см. [5], стр. 20). Первые три примера показывают, что в общем случае каждый из параметров $r_{1}, r_{4}$ может быть больше другого.

Пример 1. Пусть $R$ - такое кольцо, что $R \cong R^{2}, u=(1,0,0, \ldots) \in R^{\langle 1\rangle}$. Тогда $R[x] u=R u \cong R$, откуда $r_{1}=r_{2}=r_{3}=1$. Очевидно, $r_{5}=r_{6}=1$. С другой стороны,

$$
R[x] u \cong R \cong R^{2} \cong R^{n}
$$

для произвольного $n$. Следовательно, в $R[x] u$ можно найти $n$ линейно независимых над $R$ элементов. Отсюда, $r_{4}=\infty$. В этом примере

$$
1=r_{1}=r_{2}=r_{3}=r_{5}=r_{6}<r_{4}=\infty \text {. }
$$

Чтобы сделать пример более конкретным, отметим, что в качестве $R$ можно взять кольцо линейных преобразований бесконечномерного векторного пространства над полем. Например, пусть $V$ - счетномерное пространство над полем $P$, элементы которого мы рассматриваем как бесконечные векторы столбцы, и пусть $R=\operatorname{End}(V)$. Тогда $R$ можно рассматривать как кольцо матриц над $P$ со счетным числом строк и столбцов таких, что в каждом столбце находится лишь конечное число ненулевых элементов. В качестве $n$ линейно независимых элементов рассмотренного выше модуля сдвигов $R[x] u$ можно взять последовательности

$$
v_{t}=\left(v_{t}(0), 0,0, \ldots\right), \quad t=1, \ldots, n,
$$

где $v_{t}(0)-$ матрица

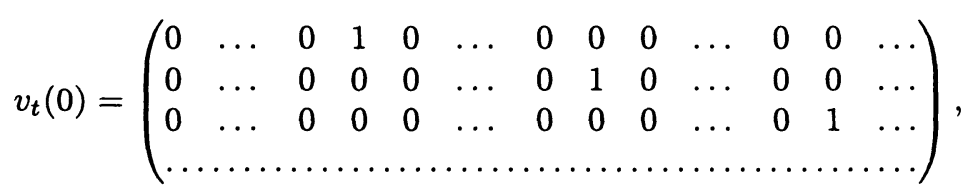

у которой в $i$-й строке, $i \geqslant 0$, находится одна единица, перед которой стоит $(t-1)+n i$ нулей.

Пример 2. Пусть $R \cong R^{2}, u=(0,1,0,0, \ldots) \in R^{\langle 1\rangle}$. Тогда $R[x] u={ }_{R}(u, x u) \cong R^{2}$. Легко видеть, что $r_{2}=r_{3}=2, r_{5}=r_{6}=2$. Как и в примере 1 , показывается, что $r_{4}=\infty$. Так как $R^{2} \cong R$, то $R$-модуль $R[x] u$ порождается одним элементом, откуда $r_{1}=1$. В этом примере

$$
1=r_{1}<2=r_{2}=r_{3}=r_{5}=r_{6}<r_{4}=\infty .
$$

Если $R=\operatorname{End}(V)-$ кольцо из примера 1 , то

$$
R[x] u={ }_{R}(v),
$$

где $v=(v(0), v(1), 0,0, \ldots)$,

$$
v(0)=\left(\begin{array}{cccc}
1 & 0 & 0 & \ldots \\
0 & 0 & 0 & \ldots \\
0 & 1 & 0 & \ldots \\
0 & 0 & 0 & \ldots \\
0 & 0 & 1 & \ldots \\
0 & 0 & 0 & \ldots \\
\ldots & \ldots & \ldots
\end{array}\right), \quad v(1)=\left(\begin{array}{cccc}
0 & 0 & 0 & \ldots \\
1 & 0 & 0 & \ldots \\
0 & 0 & 0 & \ldots \\
0 & 1 & 0 & \ldots \\
0 & 0 & 0 & \ldots \\
0 & 0 & 1 & \ldots \\
\ldots & \ldots
\end{array}\right)
$$


Действительно, $v \in R[x] u$ и $u=a v, x u=b v$ для элементов

$$
a=\left(\begin{array}{ccccccc}
0 & 1 & 0 & 0 & 0 & 0 & \ldots \\
0 & 0 & 0 & 1 & 0 & 0 & \ldots \\
0 & 0 & 0 & 0 & 0 & 1 & \ldots \\
\ldots & \ldots & \ldots & \ldots
\end{array}\right), \quad b=\left(\begin{array}{ccccccc}
1 & 0 & 0 & 0 & 0 & 0 & \ldots \\
0 & 0 & 1 & 0 & 0 & 0 & \ldots \\
0 & 0 & 0 & 0 & 1 & 0 & \ldots \\
\ldots & \ldots & \ldots
\end{array}\right)
$$

кольца $R$.

В рассмотренных примерах $r_{1}<r_{4}$. Как будет показано в предложении 7 , если $R$ коммутативно или артиново слева, то $r_{1} \geqslant r_{4}$. Следующий пример показывает возможность неравенства $r_{1}>r_{4}$.

Пример 3. Пусть $R=Z_{4}, u=(2,2,2, \ldots) \in R^{\langle 1\rangle}$. Тогда

$$
r_{1}=r_{2}=r_{3}=1>r_{4}=r_{5}=r_{6}=0 \text {. }
$$

Данный пример можно обобщить. Пусть $M$ - модуль с кручением и $a \in M-$ периодический элемент, то есть $a \neq 0$ и $r a=0$ для некоторого $r \in R \backslash 0$. Частным случаем является ситуация, когда $R=M-$ кольцо с делителями нуля и $a-$ правый делитель нуля. Тогда для 1-последовательности $u=(a, a, a, \ldots)$ выполняются соотношения $r_{1}=r_{2}=r_{3}=1, r_{4}=r_{5}=r_{6}=0$.

Согласно примерам 1,2 , неравенства $r_{1} \leqslant r_{2}$ и $r_{4} \geqslant r_{5}$ из (1) могут быть строгими. Покажем, что это возможно и в коммутативном случае.

Пример 4. Пусть $R=\mathrm{Z}$ или $R=Z_{6}, u \in R^{\langle 2\rangle}-2$-последовательность вида

$$
u=\longdiv { \begin{array} { l l } 
{ 1 } & { 2 } \\
{ 3 }
\end{array} }
$$

то есть $u(0,0)=1, u(1,0)=2, u(0,1)=3$, и все остальные знаки $u$ равны 0 . Тогда

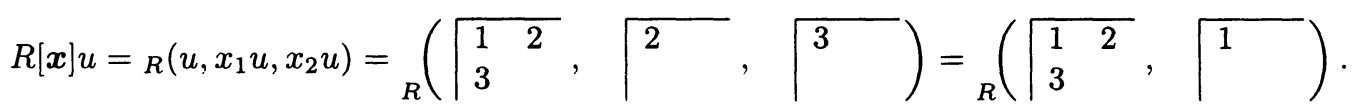

Следовательно, $r_{1}=2, r_{2}=r_{3}=3$. Кроме того, если $R=\mathbf{Z}$, то $r_{4}=r_{5}=r_{6}=2$, а если $R=Z_{6}$, то $r_{4}=2, r_{5}=r_{6}=1$.

Найдем $r_{7}, r_{8}$ и $r_{9}$. Так как

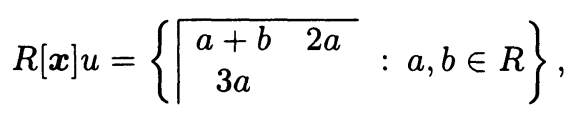

то $R[\boldsymbol{x}] u \cong R^{2}$ и $r_{7}=2$. Кроме того, мы видим, что при $R=\mathbf{Z}$ диаграмма Ферре $\mathscr{F}=\{(0,0),(1,0)\}$ является определяющей в смысле определений $r_{8}$ и $r_{9}$, поскольку по диаграмме значений $v[\mathscr{F}]$ однозначно определяются элементы $a, b$ и последовательность $v \in R[\boldsymbol{x}] u$. Отсюда легко следует, что $r_{8}=r_{9}=2$. Если же $R=Z_{6}$, то множества

$$
\Omega=\{(0,0),(1,0)\}, \quad\{(0,0),(0,1)\}, \quad\{(1,0),(0,1)\}
$$

не являются определяющими в смысле определений $r_{8}$ и $r_{9}$, поскольку существует ненулевая последовательность $v \in R[\boldsymbol{x}] u$ такая, что $v[\Omega]=0$. Отсюда следует, что $r_{8}=r_{9}=3$. В итоге, если $R=\mathbf{Z}$, то

$$
r_{1}=2, \quad r_{2}=r_{3}=3, \quad r_{4}=r_{5}=r_{6}=r_{7}=r_{8}=r_{9}=2,
$$


а если $R=Z_{6}$, то

$$
r_{1}=2, \quad r_{2}=r_{3}=3, \quad r_{4}=2, \quad r_{5}=r_{6}=1, \quad r_{7}=2, \quad r_{8}=r_{9}=3 .
$$

Найдем $r_{10}, \ldots, r_{15}$. Так как $u$ есть выход 2-ЛРС $\langle\chi, \mathscr{F}\rangle$, где

$$
\mathscr{F}=\{(0,0),(1,0),(0,1)\}, \quad \chi=\left\{x_{1}^{2}, x_{1} x_{2}, x_{2}^{2}\right\}
$$

то $r_{11}(u) \leqslant 3$. Рассматривая ту же диаграмму Ферре $\mathscr{F}$ и $\mathscr{F}$-ЛРС-систему $\left(\varphi_{1}, \varphi_{2}\right)$ из двух скалярных эндоморфизмов

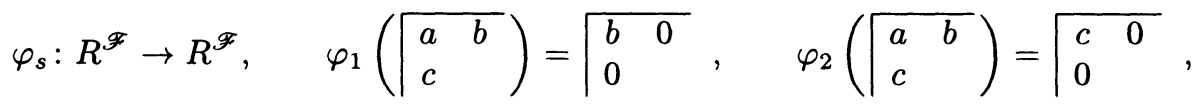

получим, что $r_{15} \leqslant 3$. Ввиду $(7) r_{i} \leqslant 3, i=12,13,14,15$. Непосредственная проверка того, что $r_{i}=3, i=10, \ldots, 15$, является весьма громоздкой, поэтому воспользуемся результатами, которые будут доказаны позже. По предложению $8 r_{12}=r_{13}$ и по предложениям 4(b) и $3 r_{13} \geqslant r_{10}=r_{3}=3$. Следовательно, ввиду (7), $r_{i}=3, i=$ $12,13,14,15$. По предложению $6(\mathrm{~d}) r_{11} \geqslant r_{14}$, откуда, $r_{11}=3$. Таким образом, в обоих случаях $\left(R=\mathbf{Z}\right.$ и $\left.R=Z_{6}\right)$

$$
r_{10}=r_{11}=r_{12}=r_{13}=r_{14}=r_{15}=3 \text {. }
$$

Помимо примера неравенств $r_{1}<r_{2}$ и $r_{4}>r_{5}$ мы увидели, что значения $r_{8}, r_{9}$ могут быть больше, чем $r_{1}, r_{4}$. В следующем примере $r_{7}, r_{8}, r_{9}$ меньше, чем $r_{1}, r_{4}$.

Пример 5. Пусть $R$ - поле, $\alpha, \beta \in R, \alpha \neq \beta$,

$$
u(i)=\left(\begin{array}{c}
\alpha^{i} \\
\beta^{i}
\end{array}\right), \quad i \geqslant 0,
$$

есть 1-последовательность над модулем $M=R^{2}$. Ее минимальный многочлен равен $(x-\alpha)(x-\beta)$, поэтому $r_{10}=2$. Модуль сдвигов $R[x] u={ }_{R}(u, x u)$ есть векторное пространство размерности 2 , откуда легко следует, что

$$
r_{1}=r_{2}=r_{3}=r_{4}=r_{5}=r_{6}=2 \text {. }
$$

При этом

$$
R[x] u=\{a u+b x u: a, b \in R\}
$$

и если $v=a u+b x u$, то

$$
v(0)=\left(\begin{array}{l}
a+b \alpha \\
a+b \beta
\end{array}\right)
$$

Так как по значению $v(0)$ можно однозначно определить константы $a, b$ и всю последовательность $v \in R[x] u$, множество $\Omega=\{0\}$ удовлетворяет определениям $r_{8}$ и $r_{9}$. Отсюда, $r_{8}=r_{9}=1$. В силу (1) $r_{7}=1$. Таким образом, $r_{7}, r_{8}, r_{9}$ меньше, чем $r_{1}$, $r_{4}$.

В этом примере интересно найти $r_{11}, \ldots, r_{15}$. Как будет показано ниже (предложения $12(\mathrm{c})$ и $13(\mathrm{~d})), r_{11}, r_{13}$ и $r_{15}$ равны $r_{1}$ и, следовательно, равны 2 . Пусть $\mathscr{F}=\{0\}$. Тогда $M^{\mathscr{F}}=M=R^{2}$. Непосредственно видно, что эндоморфизм $\varphi_{1} \in$ $\operatorname{End}\left(M^{\mathscr{F}}\right), \varphi_{1}: R^{2} \rightarrow R^{2}$, задаваемый правилом

$$
\varphi_{1}\left(\begin{array}{l}
a \\
b
\end{array}\right)=\left(\begin{array}{ll}
\alpha & 0 \\
0 & \beta
\end{array}\right)\left(\begin{array}{l}
a \\
b
\end{array}\right)=\left(\begin{array}{c}
\alpha a \\
\beta b
\end{array}\right)
$$


образует $\mathscr{F}$-ЛРС систему, удовлетворяющую определению $r_{14}$, то есть

$$
u(i)=\varphi_{1}^{i}(u(0)), \quad i \geqslant 0 .
$$

Следовательно, $r_{14}=1$, и в силу (7) $r_{12}=1$. В итоге

$$
\begin{gathered}
r_{1}=r_{2}=r_{3}=r_{4}=r_{5}=r_{6}=2, \quad r_{7}=r_{8}=r_{9}=1, \\
r_{10}=r_{11}=r_{13}=r_{15}=2, \quad r_{12}=r_{14}=1
\end{gathered}
$$

Другие примеры будут приводиться в тексте работы. Отметим, что в разделе 13 приводится общая таблица всех рассмотренных примеров.

\section{6. Общий случай}

Предложение 3. Справедливо равенство $r_{3}=r_{10}$.

Доказателъство. Если существует полная система $\mathscr{F}$-унитарных многочленов из $\operatorname{An}(u)$, то модуль операторов $R[\boldsymbol{x}] / \operatorname{An}(u)$ порождается элементами $\boldsymbol{x}^{i}+\operatorname{An}(u), i \in$ $\mathscr{F}$. Очевидно, что верно и обратное утверждение. Поэтому определение $r_{10}$ можно записать следующим образом:

$$
r_{10}=\min \left\{|\mathscr{F}|: R[x] / \operatorname{An}(u)={ }_{R}\left(x^{i}+\operatorname{An}(u), i \in \mathscr{F}\right)\right\}
$$

В силу следствия $1 r_{3}=r_{10}$.

В частности, если $k=1$, то ввиду $(2) r_{3}(u)=r_{10}(u)=\operatorname{rank} u$ есть степень минимального многочлена ЛРП $u$.

Предложение 4. Пусть $u \in \mathscr{L}_{R} M^{\langle k\rangle}$. Тогда справедливы следующие соотношения:

(a) $r_{9} \leqslant r_{12}$

(b) $r_{10} \leqslant r_{13}$;

(c) $r_{11} \leqslant r_{15}$;

(d) если $R$ конечно, то $r_{4} \leqslant r_{18} \leqslant r_{1}, r_{18} \leqslant r_{7} \log _{|R|}|M|$.

Доказателъство. Докажем утверждение (а). Пусть $\mathscr{F}$ и $\varphi=\left(\varphi_{1}, \ldots, \varphi_{k}\right)$ удовлетворяют условиям определения $r_{12}$. Достаточно доказать, что $\mathscr{F}$ удовлетворяет условиям определения $r_{9}$, то есть, что

$$
R[\boldsymbol{x}] u \rightarrow M^{\mathscr{F}}, \quad v \rightarrow v[\mathscr{F}]
$$

- мономорфизм. Для этого предположим, что

$$
v=H(\boldsymbol{x}) u \in R[\boldsymbol{x}] u, \quad v[\mathscr{F}]=0 .
$$


Тогда для любого $i \in \mathbf{N}_{0}^{k}$

$$
\begin{aligned}
v[\boldsymbol{i}+\mathscr{F}] & =(H(\boldsymbol{x}) u)[\boldsymbol{i}+\mathscr{F}]=\left(\sum_{\boldsymbol{j}} h_{\boldsymbol{j}} \boldsymbol{x}^{\boldsymbol{j}} u\right)[\boldsymbol{i}+\mathscr{F}] \\
& =\sum_{\boldsymbol{j}} h_{\boldsymbol{j}} u[\boldsymbol{i}+\boldsymbol{j}+\mathscr{F}]=\sum_{\boldsymbol{j}} h_{\boldsymbol{j}} \varphi^{\boldsymbol{i}+\boldsymbol{j}}(u[\mathscr{F}]) .
\end{aligned}
$$

Так как $\varphi_{1}, \ldots, \varphi_{k}$ - эндоморфизмы $R$-модуля $M^{\mathscr{F}}$, они перестановочны с элементами $h_{j}$. Следовательно,

$$
\begin{aligned}
v[i+\mathscr{F}] & =\varphi^{i}\left(\sum_{j} h_{j} \varphi^{j}(u[\mathscr{F}])\right) \\
& =\varphi^{i}\left(\sum_{j} h_{j} u[j+\mathscr{F}]\right)=\varphi^{i}\left(\left(\sum_{j} h_{j} x^{j} u\right)[\mathscr{F}]\right) \\
& =\varphi^{i}((H(x) u)[\mathscr{F}])=\varphi^{i}(v[\mathscr{F}])=\varphi^{i}(0)=0 .
\end{aligned}
$$

Таким образом, $v=0$, что и требовалось доказать.

Докажем утверждение (b). Пусть $\mathscr{F}$ и $\varphi=\left(\varphi_{1}, \ldots, \varphi_{k}\right)$ удовлетворяют условиям определения $r_{13}$. Достаточно доказать, что $\mathscr{F}$ удовлетворяет условиям определения $r_{10}$. Для этого по скалярным эндоморфизмам $\varphi_{1}, \ldots, \varphi_{k} \in \widehat{\operatorname{End}}\left(M^{\mathscr{F}}\right)$ построим полную систему $\mathscr{F}$-унитарных многочленов из $\operatorname{An}(u)$. Построение в основном заключается во введении обозначений. Эндоморфизм $\varphi_{s}, s=1, \ldots, k$, запишем в виде $\varphi_{s}=\left(\varphi_{s j}, j \in \mathscr{F}\right)$, где $\varphi_{s j}: M^{\mathscr{F}} \rightarrow M-$ компоненты $\varphi_{s}$. Тогда

$$
\varphi_{s}(\delta[\mathscr{F}])(\boldsymbol{j})=\varphi_{s \boldsymbol{j}}(\delta[\mathscr{F}]) \in M, \quad \delta[\mathscr{F}] \in M^{\mathscr{F}} .
$$

Так как эндоморфизм $\varphi_{s}$ скалярный, существуют коэффициенты $h_{s j l} \in R$ такие, что

$$
\varphi_{s j}(\delta[\mathscr{F}])=\sum_{\boldsymbol{l} \in \mathscr{F}} h_{s j l} \delta(l)
$$

Пусть $\boldsymbol{r} \in \Delta \mathscr{F}$. Выберем пару $(s, \boldsymbol{j}), s=1, \ldots, k, \boldsymbol{j} \in \mathscr{F}$, такую, что $\mathbf{1}_{s}+\boldsymbol{j}=\boldsymbol{r}$. Если таких пар несколько, выберем любую из них. Положим

$$
H_{\boldsymbol{r}}(\boldsymbol{x})=\boldsymbol{x}^{\boldsymbol{r}}-\sum_{\boldsymbol{l} \in \mathscr{F}} h_{s \boldsymbol{j} \boldsymbol{l}} \boldsymbol{x}^{\boldsymbol{l}}, \quad \boldsymbol{r} \in \Delta \mathscr{F}
$$

Тогда $\left\{H_{\boldsymbol{r}}(\boldsymbol{x}): \boldsymbol{r} \in \Delta \mathscr{F}\right\}-$ полная система $\mathscr{F}$-унитарных многочленов (естественным образом определяемая скалярными эндоморфизмами $\varphi$ ). Докажем, что $H_{\boldsymbol{r}}(\boldsymbol{x}) \in$ $\operatorname{An}(u)$. Пусть $(s, j)$ - пара, выбранная выше для данного $\boldsymbol{r} \in \Delta \mathscr{F}$. Тогда для любого $i \in \mathbf{N}_{0}^{k}$

$$
u\left[1_{s}+i+\mathscr{F}\right]=\varphi^{1_{s}+i}(u[\mathscr{F}])=\varphi^{1_{s}}\left(\varphi^{i}(u[\mathscr{F}])\right)=\varphi_{s}(u[i+\mathscr{F}]) .
$$

Теперь согласно (9)

$$
u\left(\mathbf{1}_{s}+\boldsymbol{i}+\boldsymbol{j}\right)=\varphi_{s \boldsymbol{j}}(u[\boldsymbol{i}+\mathscr{F}])
$$


и в силу (10)

$$
u(i+r)=u\left(1_{s}+i+j\right)=\sum_{l \in \mathscr{F}} h_{s j l} u(i+l), \quad i \in \mathbf{N}_{0}^{k} .
$$

Это и означает, что $H_{\boldsymbol{r}}(\boldsymbol{x}) \in \operatorname{An}(u)$.

Докажем (c). Пусть $\mathscr{F}$ и $\varphi=\left(\varphi_{1}, \ldots, \varphi_{k}\right)$ удовлетворяют условиям определения $r_{15}$. Достаточно доказать, что $\mathscr{F}$ удовлетворяет условиям определения $r_{11}$, то есть, что существует ЛРС $\langle\chi, \mathscr{F}\rangle$, выходом которого является ЛРП $u$. Пусть $\chi=\left\{H_{\boldsymbol{r}}(\boldsymbol{x}), \boldsymbol{r} \in \Delta \mathscr{F}\right\}-$ полная система $\mathscr{F}$-унитарных многочленов из $\mathrm{An}(u)$, естественным образом определяемая скалярными эндоморфизмами $\varphi_{1}, \ldots, \varphi_{k}$ и построенная в (b). Так как $\chi \subset \operatorname{An}(u)$, то $u \in L_{M}(\chi)$. Остается доказать, что $\langle\chi, \mathscr{F}\rangle$ есть ЛРС, то есть, что отображение $\sigma$, определенное в (3), биективно. Предположим, что

$$
v_{1}, v_{2} \in L_{M}(\chi), \quad v_{1}[\mathscr{F}]=v_{2}[\mathscr{F}]
$$

Тогда, ввиду определения множества $\chi$, очевидно, что $v_{1}(i)=v_{2}(i), i \in \mathbf{N}_{0}^{k}$, то есть $v_{1}=v_{2}$, и $\sigma$ инъективно. Для произвольной диаграммы значений $\delta[\mathscr{F}] \in M^{\mathscr{F}}$ определим последовательность $v \in M^{\langle k\rangle}$ правилом

$$
v[i+\mathscr{F}]=\varphi^{i}(\delta[\mathscr{F}]), \quad i \in \mathbf{N}_{0}^{k} .
$$

Так как эндоморфизмы $\varphi_{1}, \ldots, \varphi_{k}$ образуют $\mathscr{F}$-ЛРС систему, определение последовательности $v$ корректно. Так же, как в (b), доказывается, что $H_{\boldsymbol{r}}(\boldsymbol{x}) \in \operatorname{An}(v)$ (для данного $\boldsymbol{r}$ выбрать пару $(s, \boldsymbol{j})$ и в соотношениях (11)-(13) заменить $u$ на $v$ ). Поэтому $v \in L_{M}(\chi)$, и $\sigma$ сюръективно. Этим доказано, что $\langle\chi, \mathscr{F}\rangle-$ ЛРС с выходом $u$, и $r_{11} \leqslant r_{15}$.

Наконец, докажем утверждение (d). Если $R[\boldsymbol{x}] u={ }_{R}\left(v_{1}, \ldots, v_{n}\right), n=r_{1}$, то $|R[\boldsymbol{x}] u| \leqslant|R|^{n}$ и $r_{18} \leqslant n=r_{1}$. Если $v_{1}, \ldots, v_{n} \in R[\boldsymbol{x}] u$ линейно независимы, $n=r_{4}$, то $|R[\boldsymbol{x}] u| \geqslant|R|^{n}$ и $r_{18} \geqslant n=r_{4}$. Если $R[\boldsymbol{x}] u \rightarrow M^{t}-$ мономорфизм, $t=r_{7}$, то

$$
r_{18} \leqslant \log _{|R|}|M|^{t}=r_{7} \log _{|R|}|M| \text {. }
$$

Для 1-ЛРП над полем все неравенства в предложении 4 обращаются в равенства. Покажем, что все они могут быть строгими. В примере 4 (для случая $R=Z$ Z) $r_{9}=$ $2<r_{12}=3$. В примере 3 (для случая $R=Z_{4}$ ) $|R[x] u|=2$, откуда, $r_{18}=1 / 2$ и $0=r_{4}<r_{18}<r_{1}=1$, а также

$$
r_{18}<r_{7} \log _{|R|}|M|=r_{7}=1 \text {. }
$$

В следующем примере $r_{10}<r_{13}$ и $r_{11}<r_{15}$.

Пример 6. Пусть $b$ - элемент некоммутативного кольца $R$ такой, что $R b \nsubseteq b R$, например, $R=P_{2}$ - кольцо $2 \times 2$-матриц над полем $P$ и

$$
b=\left(\begin{array}{ll}
1 & 0 \\
0 & 0
\end{array}\right) \text {. }
$$

Выберем элемент $a \in R$ такой, что $a b \notin b R$, и положим

$$
u=\left(b, a b, \ldots, a^{i} b, \ldots\right) \in R^{\langle 1\rangle} .
$$


Тогда $u \in L_{R}(x-a)$, откуда, $r_{10}=1, r_{1}=r_{2}=r_{3}=1$. Очевидно также, что $r_{5}=r_{6}=1$ и $r_{7}=1$. Так как $u-$ выход ЛРС $\langle x-a,\{0\}\rangle$, то $r_{11}=1$. Покажем, что $r_{12}>1$. Предположим, что $r_{12}=1$. Тогда существует эндоморфизм $\varphi \in \operatorname{End}\left({ }_{R} R\right)$ такой, что

$$
u(i)=\varphi^{i}(u(0))=\varphi^{i}(b), \quad i \geqslant 0 .
$$

В частности, $u(1)=a b=\varphi(b)$. Но $\varphi(b)=b \varphi(1) \in b R$, тогда как $a b \notin b R$, получаем противоречие. Следовательно, $r_{12}>1$. В силу (7), $r_{13}, r_{14}, r_{15}>1$.

Предложение 5. Пусть модуль $M$ конечен, $|M| \leqslant|R|$ (колъцо $R$ может быть бесконечным), $u \in \mathscr{L}_{R} M^{\langle k\rangle}$. Тогда $r_{4} \leqslant r_{7}$.

Доказательство. Пусть $R[\boldsymbol{x}] u \rightarrow M^{t}-$ мономорфизм, $t=r_{7}$. Возьмем произвольные $n, n>t$, последовательностей $v_{1}, \ldots, v_{n} \in R[\boldsymbol{x}] u$. Из векторов строк $\varphi\left(v_{1}\right), \ldots, \varphi\left(v_{n}\right) \in M^{t}$ составим $n \times t$ матрицу

$$
V=\left(\begin{array}{c}
\varphi\left(v_{1}\right) \\
\ldots \\
\varphi\left(v_{n}\right)
\end{array}\right)
$$

над модулем $M$. Так как $n>t$ и $|R| \geqslant|M|$, отображение $R^{n} \rightarrow M^{t}, \boldsymbol{x} \rightarrow \boldsymbol{x} V$, являющееся гомоморфизмом $R$-модулей, не инъективно. Следовательно, $c V=0$ для некоторого

$$
\boldsymbol{c}=\left(c_{1}, \ldots, c_{n}\right) \in R^{n} \backslash \mathbf{0}
$$

Это означает, что

$$
c_{1} \varphi\left(v_{1}\right)+\ldots+c_{n} \varphi\left(v_{n}\right)=0
$$

то есть

$$
\varphi\left(c_{1} v_{1}+\ldots+c_{n} v_{n}\right)=0 .
$$

Так как $\varphi-$ мономорфизм, то $c_{1} v_{1}+\ldots+c_{n} v_{n}=0$. Таким образом, любые $n>t$ последовательностей из $R[\boldsymbol{x}] u$ линейно зависимы. Следовательно, $r_{4} \leqslant t=r_{7}$.

В условиях предложения 5 неравенство $r_{4} \leqslant r_{7}$ может быть строгим (пример 3 и примеры 7, 11 ниже), и может обращаться в равенство (пример 4 и пример 12 ниже). Если условия предложения 5 нарушены, то неравенство $r_{4} \leqslant r_{7}$ может быть неверным (примеры $1,2,5$ ).

\section{7. Коммутативный случай}

Предложение 6. Пусть колъцо $R$ коммутативно, $u \in \mathscr{L}_{R} M^{\langle k\rangle}$. Тогда
(a) $r_{2} \geqslant r_{8}$
(b) $r_{3} \geqslant r_{9}$;
(c) $r_{9} \leqslant r_{11}$
(d) $r_{11} \geqslant r_{14}$
(e) $r_{13} \leqslant\left(r_{12} \nu\right)^{k}$, где $\nu=r_{1}(M)$; 
(f) $r_{16} \leqslant r_{11} \nu$

Доказательство. Докажем (а). Пусть $\Omega$ удовлетворяет определению $r_{2}$, то есть $R[\boldsymbol{x}] u={ }_{R}\left(\boldsymbol{x}^{i} u, \boldsymbol{i} \in \Omega\right)$. Тогда для любого $\boldsymbol{r} \in \mathbf{N}_{0}^{k} \backslash \Omega$ последовательность $\boldsymbol{x}^{\boldsymbol{r}} u$ линейно выражается через $\left(\boldsymbol{x}^{i} u, i \in \Omega\right)$. Это означает, что существует многочлен $H_{\boldsymbol{r}}(\boldsymbol{x}) \in \operatorname{An}(u)$ вида

$$
H_{r}(x)=x^{r}-\sum_{i \in \Omega} h_{r i} x^{i}
$$

Возьмем теперь произвольную последовательность $v \in R[\boldsymbol{x}] u$ такую, что $v[\Omega]=0$. Так как $R$ коммутативно, из условия $H_{\boldsymbol{r}}(\boldsymbol{x}) u=0$ следует, что $H_{\boldsymbol{r}}(\boldsymbol{x}) v=0$. Так как $v[\Omega]=0$, то $v(\boldsymbol{r})=0, \boldsymbol{r} \in \mathbf{N}_{0}^{k} \backslash \Omega$, то есть $v=0$. Таким образом, $\Omega$ удовлетворяет определению $r_{8}$. Отсюда следует неравенство $r_{2} \geqslant r_{8}$.

Доказательство (b) получается из доказательства (a) заменой $\Omega$ на $\mathscr{F}$.

Докажем (c). Пусть $\mathscr{F}$ удовлетворяет определению $r_{11}$, то есть $u-$ выход ЛРС $\langle\chi, \mathscr{F}\rangle$. Тогда $u \in L_{M}(\chi)$ и отображение $\sigma: L_{M}(\chi) \rightarrow M^{\mathscr{F}}, v \rightarrow v[\mathscr{F}]$, определенное в (3), биективно. Так как $R$ коммутативно, то $R[\boldsymbol{x}] u \subseteq L_{M}(\chi)$. Следовательно, отображение $R[\boldsymbol{x}] u \rightarrow M^{\mathscr{F}}, v \rightarrow v[\mathscr{F}]$, инъективно, то есть $\mathscr{F}$ удовлетворяет определению $r_{9}$. Из доказанного следует, что $r_{9} \leqslant r_{11}$.

Докажем (d). Как и при доказательстве утверждения (c), пусть $\mathscr{F}$ удовлетворяет определению $r_{11}$. Так как $R$ коммутативно, $\sigma$ является изоморфизмом $R$-модулей. Для $s=1, \ldots, k$ определим отображение $\varphi_{s}: M^{\mathscr{F}} \rightarrow M^{\mathscr{F}}$, полагая $\varphi_{s}=\sigma \widehat{x}_{s} \sigma^{-1}$, где

$$
\widehat{x}_{s}: L_{M}(\chi) \rightarrow L_{M}(\chi), \quad v \rightarrow x_{s} v
$$

Другими словами, если $\delta[\mathscr{F}] \in M^{\mathscr{F}}$ и $\delta[\mathscr{F}]=\sigma(v)$ (то есть $\delta[\mathscr{F}]=v[\mathscr{F}]$ ), где $v \in$ $L_{M}(\chi)$, то мы полагаем

$$
\varphi_{s}(\delta[\mathscr{F}])=\left(x_{s} v\right)[\mathscr{F}]=v\left[\mathbf{1}_{s}+\mathscr{F}\right] .
$$

Докажем, что $\mathscr{F}$ и $\varphi=\left(\varphi_{1}, \ldots, \varphi_{k}\right)$ удовлетворяют определению $r_{14}$. Так как $\sigma$, $\widehat{x}_{s}$ и $\sigma^{-1}$ - гомоморфизмы $R$-модулей, то $\varphi_{s}$ - гомоморфизм $R$-модулей и $\varphi_{s} \in$ $\operatorname{End}\left(M^{\mathscr{F}}\right)$. Так как $\widehat{x}_{s} \widehat{x}_{t}=\widehat{x}_{t} \widehat{x}_{s}$, то $\varphi_{s} \varphi_{t}=\varphi_{t} \varphi_{s}$. Проверим условие (5). Пусть

$$
\delta[\mathscr{F}] \in M^{\mathscr{F}}, \quad s \in\{1, \ldots, k\}, \quad j \in \mathscr{F}, \quad j+\mathbf{1}_{s} \in \mathscr{F},
$$

и пусть $\varphi_{s}(\delta[\mathscr{F}])=\lambda[\mathscr{F}]$. Выберем $v \in L_{M}(\chi)$ так, что $\sigma(v)=\delta[\mathscr{F}]$. Тогда $\varphi_{s}(\delta[\mathscr{F}])=$ $v\left[\mathbf{1}_{s}+\mathscr{F}\right]$, то есть $\lambda[\mathscr{F}]=v\left[\mathbf{1}_{s}+\mathscr{F}\right]$. Следовательно, $\lambda(\boldsymbol{j})=v\left(\mathbf{1}_{s}+j\right)=\delta\left(\mathbf{1}_{s}+j\right)$, что доказывает (5). Таким образом, $\varphi$ есть $\mathscr{F}$-ЛРС система. Теперь проверим условие

$$
u[i+\mathscr{F}]=\varphi^{i}(u[\mathscr{F}]), \quad i \in \mathbf{N}_{0}^{k},
$$

из определения $r_{14}$. Очевидно, достаточно убедиться в том, что из равенства $u[i+\mathscr{F}]=\varphi^{i}(u[\mathscr{F}])$ следует равенство

$$
u\left[\mathbf{1}_{s}+i+\mathscr{F}\right]=\varphi^{1_{s}+i}(u[\mathscr{F}]) .
$$

Действительно,

$$
\begin{aligned}
\varphi^{1_{s}+\boldsymbol{i}}(u[\mathscr{F}]) & =\varphi_{s}\left(\varphi^{i}(u[\mathscr{F}])\right)=\varphi_{s}(u[i+\mathscr{F}])=\sigma \widehat{x}_{s} \sigma^{-1}(u[i+\mathscr{F}]) \\
& =\sigma \widehat{x}_{s} \sigma^{-1}\left(\left(x^{i} u\right)[\mathscr{F}]\right)=\sigma \widehat{x}_{s}\left(x^{i} u\right)=\sigma\left(x_{s} \boldsymbol{x}^{i} u\right) \\
& =\sigma\left(\boldsymbol{x}^{1_{s}+i} u\right)=\left(\boldsymbol{x}^{1_{s}+i} u\right)[\mathscr{F}]=u\left[\mathbf{1}_{s}+\boldsymbol{i}+\mathscr{F}\right] .
\end{aligned}
$$


Тем самым мы доказали, что $\mathscr{F}, \varphi$ удовлетворяют определению $r_{14}$, откуда следует неравенство $r_{11} \geqslant r_{14}$.

Это неравенство (е) доказано в предложении 1.4 в [6].

Наконец, докажем утверждение (f). Пусть $u$ является выходом ЛРС $\langle\chi, \mathscr{F}\rangle,|\mathscr{F}|=$ $r_{11}$. Тогда $u \in L_{M}(\chi) \cong M^{\mathscr{F}}$. Так как $R$ коммутативно, то $R[\boldsymbol{x}] u \subseteq L_{M}(\chi)$. Следовательно,

$$
r_{16} \leqslant r_{1}\left(M^{\mathscr{F}}\right) \leqslant|\mathscr{F}| r_{1}(M)=r_{11} \nu
$$

Каждый из примеров 4 (для случая $R=\mathrm{Z}$ ), 5 и пример 8 ниже показывают, что неравенства $r_{2} \geqslant r_{8}, r_{3} \geqslant r_{9}, r_{9} \leqslant r_{11}$ могут быть строгими. Согласно примеру 5 неравенство $r_{11} \geqslant r_{14}$ также может быть строгим. Следующий пример показывает, что неравенство $r_{16} \leqslant r_{11} \nu$ может быть строгим.

Пример 7. Пусть $P$ - поле, $P(\alpha, \beta)$ - трансцендентное расширение степени трансцендентности $2, R=P(\alpha, \beta) /\left(\alpha^{2}, \beta^{2}\right)$. Тогда $R$ является коммутативным локальным кольцом, конечным если $P$ конечно, и алгеброй над $P$. Рассмотрим последовательность

$$
u=(0, \alpha \beta, 0,0, \ldots) \in R^{\langle 1\rangle} .
$$

Тогда $u \in L_{R}\left(x^{2}\right)$ и $r_{11}=2$. При этом $R[x] u \subseteq R(v)$, где $v=(\alpha, \beta, 0,0, \ldots)$, поскольку $u=\alpha v, x u=\beta v$. Следовательно, $r_{16}=1$ и $r_{16}<r_{11}$.

Условие коммутативности кольца $R$ в предложении $6(\mathrm{a}-\mathrm{d})$ является существенным. Действительно, в примере $6 r_{11}<r_{14}$, то есть неравенство из предложения $6(\mathrm{~d})$ неверно. В следующем примере $r_{2}<r_{8}, r_{3}<r_{9}$ и $r_{9}>r_{11}$, то есть неравенства из предложения 6(a-c) неверны.

Пример 8. Пусть $R=P_{2}-$ кольцо $2 \times 2$ матриц над полем $P$,

$$
a=\left(\begin{array}{ll}
0 & 1 \\
1 & 0
\end{array}\right), \quad b=\left(\begin{array}{ll}
1 & 0 \\
0 & 0
\end{array}\right),
$$

$u=\left(b, a b, \ldots, a^{i} b, \ldots\right) \in R^{\langle 1\rangle}$. Как и в примере $6, r_{1}=r_{2}=r_{3}=r_{5}=r_{6}=r_{7}=r_{10}=$ $r_{11}=1$. Предположим, что $r_{8}=1$. Тогда существует одноэлементное множество $\Omega=\{i\}$, определяющее в смысле определения $r_{8}$. Положим

$$
c=\left(\begin{array}{ll}
0 & 0 \\
0 & 1
\end{array}\right)
$$

если $i$ четно, и $c=b$, если $i$ нечетно. Тогда $c a^{i} b=0, c a^{i+1} b \neq 0$. Следовательно, две различные последовательности $c u, 0 \in R[x] u$ имеют одинаковые диаграммы значений на множестве $\Omega$, получаем противоречие. Таким образом, $r_{8}>1$. В силу (1) $r_{9} \geqslant r_{8}>1$.

Лемма 1. Если кольцо $R$ коммутативно или артиново слева, то для любого левого $R$-модуля $M$ выполняется неравенство $r_{4}(M) \leqslant r_{1}(M)$.

Доказательство. Пусть $r_{1}(M)=m$. Тогда $M$ порождается $m$ элементами и существует эпиморфизм $R$-модулей $\pi: R^{m} \rightarrow M$. Пусть $s_{1}, \ldots, s_{n}$ - произвольные $n>m$ элементов модуля $M$ и $a_{1}, \ldots, a_{n} \in R^{m}$ - их произвольные прообразы при отображении $\pi$. Рассмотрим $n \times m$ матрицу $A$ над $R$, составленную из строк $a_{1}, \ldots, a_{n}$. Если $R$ коммутативно, то, согласно [18], стр. 159 (см. также [3], следствие 1 на стр. 213), 
из условия $n>m$ следует, что система линейных уравнений $\boldsymbol{x} A=\mathbf{0}$ имеет ненулевое решение $c=\left(c_{1}, \ldots, c_{n}\right) \in R^{n}$. Если $R$ конечно, то отображение $\varphi: R^{n} \rightarrow R^{m}$, $\varphi(\boldsymbol{x})=\boldsymbol{x} A$, не инъективно, и вновь система линейных уравнений $\boldsymbol{x} A=\mathbf{0}$ имеет ненулевое решение $\boldsymbol{c}$.

Пусть $R$ артиново слева. Предположим, что отображение $\varphi$ инъективно. Тогда модуль $R^{m}$ содержит подмодуль $\varphi\left(R^{n}\right)$, изоморфный $R^{n}$. Так как $R$ нетерово слева, то $R$ обладает свойством ИБЧ (см. [5], стр. 22). Поэтому $\varphi\left(R^{n}\right)$ не может совпадать с $R^{m}$. Таким образом, в модуле $R^{m}$ существует собственный подмодуль $\varphi\left(R^{n}\right)$, изоморфный $R^{n}, n>m$. Теперь легко построить строго убывающую цепочку подмодулей модуля $R^{m}$, изоморфных $R^{n}$. Но $R^{m}-$ конечно порожденный модуль над $R$, поэтому модуль $R^{m}$ артинов, получаем противоречие. Следовательно, отображение $\varphi$ не инъективно и система линейных уравнений $\boldsymbol{x} A=\mathbf{0}$ имеет ненулевое решение c. Таким образом, во всех случаях

$$
c_{1} a_{1}+\ldots+c_{n} a_{n}=0, \quad c \neq 0 .
$$

Подействовав гомоморфизмом $\pi$, получим, что $c_{1} s_{1}+\ldots+c_{n} s_{n}=0$. Тем самым доказано, что любые $n>m$ элементов модуля $M$ линейно зависимы. Следовательно, $r_{4}(M) \leqslant m=r_{1}(M)$.

Предложение 7. Если колъцо $R$ коммутативно или артиново слева,

$$
u \in \mathscr{L}_{R} M^{\langle k\rangle}
$$

mo

$$
r_{4} \leqslant r_{1}, \quad r_{4} \leqslant r_{7} \nu
$$

где $\nu=r_{1}(M)$. В частности, если модулъ $M$ циклический или $M=R$, то $r_{4} \leqslant r_{7}$.

Доказательство. Применив лемму 1 к модулю сдвигов $R[\boldsymbol{x}] u$, получим, что

$$
r_{4}(u)=r_{4}(R[\boldsymbol{x}] u) \leqslant r_{1}(R[\boldsymbol{x}] u)=r_{1}(u) .
$$

Если $r_{7}=\infty$, то неравенство $r_{1} \leqslant r_{7} \nu$ выполняется. Пусть $r_{7}=t<\infty$ и $R[\boldsymbol{x}] u \rightarrow M^{t}$ - мономорфизм. Тогда $r_{4}(R[\boldsymbol{x}] u) \leqslant r_{4}\left(M^{t}\right)$. По лемме $1 r_{4}\left(M^{t}\right) \leqslant r_{1}\left(M^{t}\right)$. Отсюда,

$$
r_{4}(u)=r_{4}(R[x] u) \leqslant r_{4}\left(M^{t}\right) \leqslant r_{1}\left(M^{t}\right) \leqslant t r_{1}(M)=r_{7} \nu .
$$

Заметим, что для конечного кольца $R$ неравенство $r_{4} \leqslant r_{1}$ следует также из предложения $4(\mathrm{~d})$. При условии, что $M$ конечен и $|M| \leqslant|R|$, в предложении 5 ранее было доказано также неравенство $r_{4} \leqslant r_{7}$.

Примеры 3, 7 показывают, что если $R$ коммутативно и конечно, $M=R, k=1$, то неравенства $r_{4} \leqslant r_{1}, r_{4} \leqslant r_{7}$ могут быть строгими. Примеры 1,2 показывают, что в некоммутативном случае эти неравенства могут быть неверными. Согласно примеру 5 , неравенство $r_{4} \leqslant r_{7} \nu$ может обращаться в равенство (в примере $5 \nu=2$, но легко привести аналогичный пример для произвольного $\nu$ ).

Объединяя неравенства $(1),(7),(8)$ и результаты предложений $3-7$, получим следующее утверждение. 
Следствие 2. Если $R$ коммутативно, $u \in \mathscr{L}_{R} M^{\langle k\rangle}, \nu=r_{1}(M)$, mо

$$
\begin{gathered}
r_{6} \leqslant r_{5} \leqslant r_{4} \leqslant r_{18} \leqslant r_{1} \leqslant r_{2} \leqslant r_{3}=r_{10} \leqslant r_{13} \leqslant\left(r_{12} \nu\right)^{k}, \\
r_{4} \leqslant r_{7} \nu, \quad r_{7} \leqslant r_{8} \leqslant r_{9} \leqslant r_{3}, \quad r_{8} \leqslant r_{2}, \\
r_{9} \leqslant r_{11} \leqslant r_{15}, \quad r_{9} \leqslant r_{12} \leqslant r_{13} \leqslant r_{15}, \quad r_{12} \leqslant r_{14} \leqslant r_{11}, \\
r_{16} \leqslant r_{1}, \quad r_{16} \leqslant r_{11} \nu, \quad r_{18} \leqslant r_{7} \log _{|R|}|M| .
\end{gathered}
$$

Следуя [6], модуль $M$ над коммутативным кольцом $R$ будем называть $E$-модулем, если $\operatorname{End}\left({ }_{R} M\right)=\widehat{\operatorname{End}}\left({ }_{R} M\right)$.

Предложение 8. Пусть колъцо $R$ коммутативно, модуль $M$ является $E$-модулем (в частности, $M=R$ ), $u \in \mathscr{L}_{R} M^{\langle k\rangle}$. Тогда

$$
r_{12}=r_{13}, \quad r_{14}=r_{15}
$$

Доказателъство. Так как $M$ является $E$-модулем, то, согласно предложению 1.3 в [6],

$$
\operatorname{End}\left(M^{\mathscr{F}}\right)=\widehat{\operatorname{End}}\left(M^{\mathscr{F}}\right)
$$

и определения $r_{12}$ и $r_{13}$, а также $r_{14}$ и $r_{15}$, совпадают.

\section{8. Случай $k=1$}

Предложение 9. Пусть $k=1, u \in \mathscr{L}_{R} M^{\langle 1\rangle}$. Тогда $r_{6} \leqslant r_{10} u r_{11} \leqslant r_{10}$.

Дохазателъство. Пусть $F(x)$ - минимальный многочлен $Л Р П ~ u, \operatorname{deg} F(x)=m=$ $r_{10}$. Тогда последовательности $u, x u, \ldots, x^{m} u$ линейно зависимы, откуда, $r_{6} \leqslant r_{10}$. Кроме того, последовательность $u$ является выходом $\operatorname{JPC~}\langle F(x),\{0, \ldots, m-1\}\rangle$, откуда, $r_{11} \leqslant r_{10}$.

Как показывают примеры 3,7 и пример 12 ниже, неравенство $r_{6} \leqslant r_{10}$ может быть строгим. Критерий того, что это неравенство (при $k=1)$ обращается в равенство, дан ниже в предложении 10. В силу предложения 7 (а также предложения 3 и соотношений (1)) неравенство $r_{6} \leqslant r_{10}$ выполняется и при $k>1$, если $R$ коммутативно или артиново слева; доказательство следует из неравенств

$$
r_{6} \leqslant r_{5} \leqslant r_{4} \leqslant r_{1} \leqslant r_{2} \leqslant r_{3}=r_{10}
$$

Вопрос о том, выполняется ли оно в общем случае, открыт.

При $k>1$ может выполняться как неравенство $r_{11}<r_{10}$, так и неравенство $r_{11}>r_{10}$. В [17] приведен пример 2-ЛРС-последовательности, не являющейся ЛРП. Этот пример построен В. Т. Марковым, в нем $r_{11}=3<r_{10}=\infty$. В следующем примере $r_{11}>r_{10}$.

Пример 9. Пусть $R=P\langle\alpha, \beta, \gamma\rangle / I$, где $P$ - поле, $\alpha, \beta, \gamma$ - свободные некоммутирующие переменные, $I$ - двусторонний идеал в свободной алгебре $P\langle\alpha, \beta, \gamma\rangle$, порожденный элементами $\left(\alpha^{i} \beta-\beta \alpha^{i}\right) \beta^{j} \gamma, i, j \geqslant 0$. Пусть $u \in R^{\langle 2\rangle}-2$-последовательность со знаками $u(i, j)=\alpha^{i} \beta^{j} \gamma$. Тогда $x_{1}-\alpha, x_{2}-\beta \in \operatorname{An}(u)$, откуда, $r_{10}=1$. Предположим, что $r_{11}=1$. Тогда $u$ есть выход ЛРC $\langle\chi,\{(0,0)\}\rangle$, где $\chi \subset R[\boldsymbol{x}]$ - такое множество, 
что отображение $L_{R}(\chi) \rightarrow R, v \rightarrow v(0,0)$, биективно. Пусть $v \in L_{R}(\chi)$ - такая последовательность, что $v(0,0)=1$. Введем обозначения $v(1,0)=p, v(0,1)=q$. Наша цель заключается в том, чтобы найти $p, q$ и $v(1,1)$. Заметим, что если $w \in L_{R}(\chi)$, $w(0,0)=r \in R$, то последовательности $w, v r \in L_{R}(\chi)$ имеют одинаковую диаграмму значений на множестве $\{(0,0)\}$ и по определению ЛРС $w=v r$. Взяв в качестве $w$ последовательность $u$, получим, что $u=v \gamma$. Следовательно,

$$
(u(0,0), u(1,0), u(0,1))=(\gamma, \alpha \gamma, \beta \gamma)=(\gamma, p \gamma, q \gamma)
$$

Отсюда, $p=\alpha, q=\beta$. Теперь возьмем в качестве $w$ последовательности $x_{1} v$ и $x_{2} v$. Так как

$$
\left(x_{1} v\right)(0,0)=v(1,0)=p=\alpha, \quad\left(x_{2} v\right)(0,0)=v(0,1)=q=\beta
$$

мы получим, что $x_{1} v=v \alpha, x_{2} v=v \beta$. Отсюда,

$$
v(1,1)=\left(x_{1} v\right)(0,1)=v(0,1) \alpha=\beta \alpha
$$

и

$$
v(1,1)=\left(x_{2} v\right)(1,0)=v(1,0) \beta=\alpha \beta,
$$

то есть $\alpha \beta=\beta \alpha$, получаем противоречие. Следовательно, $r_{11}>1$.

Предложение 10. Пусть $k=1, u \in \mathscr{L}_{R} M^{\langle 1\rangle}$. Тогда следующие утвержсдения равносилъны:

(а) ЛРП и имеет единственный минимальный многочлен;

(b) $\operatorname{An}(u)$ - главный левый идеал кольца $R[x]$, порождаемый унитарным многочленом;

(с) минимальный многочлен ЛРП и является ненулевым многочленом наименъшей степени в $\operatorname{An}(u)$;

(d) $r_{6}(u)=r_{10}(u)$.

Доказательство. Докажем, что $(\mathrm{a}) \Rightarrow(\mathrm{b})$. Пусть $F(x)$ - единственный минимальный многочлен ЛРП $u$. Разделив произвольный многочлен $G(x) \in \operatorname{An}(u)$ с остатком на $F(x)$ справа, получим, что

$$
G(x)=Q(x) F(x)+r(x), \quad \operatorname{deg} r(x)<\operatorname{deg} F(x) .
$$

Если $r(x) \neq 0$, то

$$
r(x) u=G(x) u-Q(x) F(x) u=0
$$

и $F(x)+r(x)$ - минимальный многочлен ЛРП $u$, не равный $F(x)$, получаем противоречие. Следовательно, $r(x)=0$, то есть

$$
G(x)=Q(x) F(x) \in R[x] F(x) .
$$

Тем самым показано, что $\operatorname{An}(u) \subseteq R[x] F(x)$. Очевидно, что верно и обратное включение. Следовательно, $\operatorname{An}(u)=R[x] F(x)$ - главный левый идеал, порождаемый унитарным многочленом. 
Докажем, что (b) $\Rightarrow(\mathrm{c})$. Пусть $\operatorname{An}(u)=R[x] F(x)$, где $F(x)$ унитарен. Так как в левом идеале $R[x] F(x)$ нет ненулевых многочленов степени меньше $\operatorname{deg} F(x)$, то $F(x)$ является минимальным многочленом ЛРП $u$ и выполняется условие (c).

Докажем, что (c) $\Leftrightarrow(\mathrm{d})$. Пусть $r_{10}(u)=m$. Согласно (2) $m$ есть степень минимального многочлена ЛРП $u$. По предложению $9 r_{6} \leqslant m$. Условие $r_{6}=m$ равносильно тому, что последовательности $u, x u, \ldots, x^{m-1} u$ линейно независимы. Это означает, что в $\operatorname{An}(u)$ не существует ненулевого многочлена степени меньше $m$. Таким образом, условия (c) и (d) равносильны.

Докажем, наконец, что $(\mathrm{d}) \Rightarrow(\mathrm{a})$. Если $F_{1}(x)$ и $F_{2}(x)$ - два различных минимальных многочлена ЛРП $u$ степени $m$, то их разность есть ненулевой многочлен степени меньше $m$ из $\operatorname{An}(u)$.

Предложение 11. Пусть $k=1, R$ коммутативно, $u \in \mathscr{L}_{R} M^{\langle 1\rangle}$. Тогда

(a) $r_{1}=r_{2}=r_{3}=r_{10}=r_{13}=r_{15}$;

(b) $r_{17} \leqslant r_{10} \nu$, где $\nu=r_{1}(M)$.

Доказательство. Докажем (а). В силу (1) и предложения $3 r_{1} \leqslant r_{2} \leqslant r_{3}=r_{10}$, при этом согласно (2) $r_{10}$ есть степень минимального многочлена ЛРП $u$. Предположим, что $r_{1}<r_{10}$. Тогда $R[x] u={ }_{R}\left(v_{1}, \ldots, v_{n}\right)$, где $n<r_{10}$. Последовательности $x v_{1}, \ldots, x v_{n}$ линейно выражаются через последовательности $v_{1}, \ldots, v_{n}$, поэтому

$$
\left(x v_{1}, \ldots, x v_{n}\right)=\left(v_{1}, \ldots, v_{n}\right) A
$$

для некоторой матрицы $A \in R_{n}$. Легко видеть, что тогда характеристический многочлен $\chi_{A}(x)$ матрицы $A$ является характеристическим многочленом последовательностей $v_{1}, \ldots, v_{n}$, а значит, и последовательности $u$. Поскольку $\operatorname{deg} \chi_{A}(x)=n<r_{10}$, мы получили противоречие с тем, что $r_{10}$ есть степень минимального многочлена ЛРП $u$. Следовательно, $r_{1} \geqslant r_{10}$, откуда, $r_{1}=r_{2}=r_{3}=r_{10}$.

В силу предложения 4 (b) и соотношения $(7) r_{10} \leqslant r_{13} \leqslant r_{15}$. Докажем, что $r_{15} \leqslant$ $r_{10}$. Пусть $\mathscr{F}=\{0,1, \ldots, m-1\}$ удовлетворяет определению $r_{10}$, то есть существует унитарный многочлен

$$
H(x)=x^{m}-\sum_{i=0}^{m-1} h_{i} x^{i}
$$

из $\operatorname{An}(u)$. Тогда $\mathscr{F}, \varphi_{1}$, где

$$
\varphi_{1}: M^{\mathscr{F}} \rightarrow M^{\mathscr{F}}, \quad \varphi_{1}\left(a_{0}, \ldots, a_{m-1}\right)=\left(a_{1}, \ldots, a_{m-1}, \sum_{i=0}^{m-1} h_{i} a_{i}\right),
$$

удовлетворяют определению $r_{15}$ ( $\varphi_{1}$ является эндоморфизмом $R$-модуля $M^{\mathscr{F}}$ ввиду коммутативности кольца $R$ ). Следовательно, $r_{15} \leqslant r_{10}$. В итоге $r_{10}=r_{13}=r_{15}$.

Докажем (b). Пусть $F(x)$ - минимальный многочлен ЛРП $u$. Тогда

$$
u \in L_{M}(F) \cong M^{m},
$$

где $m=\operatorname{deg} F(x)=r_{10}$. Следовательно,

$$
r_{17}(u) \leqslant r_{1}\left(L_{M}(F)\right)=r_{1}\left(M^{m}\right) \leqslant m r_{1}(M)=r_{10} \nu .
$$


Следствие 3. Пусть $R$ хоммутативно, $k=1, u \in \mathscr{L}_{R} M^{\langle 1\rangle}, \nu=r_{1}(M)$. Тогда

$$
\begin{gathered}
r_{6} \leqslant r_{5} \leqslant r_{4} \leqslant r_{18} \leqslant r_{1}=r_{2}=r_{3}=r_{10}=r_{13}=r_{15}=\operatorname{rank} u \leqslant r_{12} \nu \\
r_{7} \leqslant r_{8} \leqslant r_{9} \leqslant r_{12} \leqslant r_{14} \leqslant r_{11} \leqslant r_{1}, \quad r_{4} \leqslant r_{7} \nu \\
r_{16} \leqslant r_{1}, \quad r_{16} \leqslant r_{11} \nu, \quad r_{17} \leqslant r_{10} \nu, \quad r_{18} \leqslant r_{7} \log _{|R|}|M|
\end{gathered}
$$

Если при этом $M=R$, то

$$
\begin{gathered}
r_{6} \leqslant r_{5} \leqslant r_{4} \leqslant r_{18} \leqslant r_{7} \leqslant r_{8} \leqslant r_{9} \leqslant r_{1}=r_{2}=r_{3} \\
=r_{10}=r_{11}=r_{12}=r_{13}=r_{14}=r_{15}=\operatorname{rank} u \\
r_{16} \leqslant r_{1}, \quad r_{17} \leqslant r_{1},
\end{gathered}
$$

Доказательство. Указанные соотношения вытекают из следствия 2 и предложений 9,11 . При $M=R$ нужно учесть предложение 8 и равенство $\nu=1$.

Таким образом, в случае, когда $R$ коммутативно и $k=1$, несколько значений линейной сложности имеют общее значение $m$ :

$$
m=r_{1}=r_{2}=r_{3}=r_{10}=r_{13}=r_{15}=\operatorname{rank} u .
$$

Если к тому же $M=R$, то

$$
m=r_{1}=r_{2}=r_{3}=r_{10}=r_{11}=r_{12}=r_{13}=r_{14}=r_{15}=\operatorname{rank} u
$$

Поэтому, если необходимо выделить какую-либо одну линейную сложность 1-последовательностей над коммутативным кольцом $R$, то это может быть указанное общее значение $m$.

Из предложения 10 и следствия 3 вытекает следующее утверждение.

Следствие 4. Пусть $R$ коммутативно, $k=1, u \in \mathscr{L}_{R} M^{\langle 1\rangle}$. Тогда следующие утверждения равносильны:

(а) ЛРП и имеет единственный минимальный многочлен;

(b) $r_{6}(u)=r_{10}(u)$;

(c) $r_{i}(u)=\operatorname{rank} u, i=1, \ldots, 6,10,13,15,18$.

Если при этом $M=R$, то (с) записываетсл следующим образом:

(c') $r_{i}(u)=\operatorname{rank} u, i=1, \ldots, 15,18$.

\section{9. Линейная сложность над телами и полями}

Полученные выше результаты, справедливые для ЛРП над произвольными (коммутативными) кольцами и модулями над ними, можно рассматривать как нижнюю границу набора всех свойств линейной сложности. В этом смысле свойства и соотношения для линейной сложности ЛРП над телами, полями и векторными пространствами задают верхнюю границу, к которой могут приближаться свойства линейной сложности ЛРП над различными классами колец и модулей. 
Предложение 12. Пустъ $R-$ тело, $u \in \mathscr{L}_{R} M^{\langle k\rangle}, \nu=\operatorname{dim}_{R} M \in \mathbf{N} \cup\{\infty\}, m=$ $\operatorname{dim}_{R} R[\boldsymbol{x}]$. Тогда $m<\infty$ и справедливы следующие соотношения:

(a) $m=r_{1}=r_{2}=r_{3}=r_{4}=r_{5}=r_{6}=r_{10}=r_{16} \leqslant r_{13}$;

(b) если $\nu<\infty$, то $\left.r_{7}=\right] m / \nu[$, где $] x[$ - наименьшее челое число, большее или равное $x$; если $\nu=\infty$, то $r_{7}=0$ при $m=0$ u $r_{7}=1$ при $m \geqslant 1$;

(c) если $k=1$, то ЛРП и имеет единственный минималъный многочлен $u r_{11}=m$.

Доказательство. Докажем (a). Пусть $\Pi=\Pi(\boldsymbol{m})$ - начальный параллелепипед ЛРП $u$. Тогда

$$
R[\boldsymbol{x}] u={ }_{R}\left(\boldsymbol{x}^{i} u, i \in \Pi\right) .
$$

Следовательно, $R[\boldsymbol{x}] u$ - конечно порожденное (левое) векторное пространство. Обозначим его размерность через $m, m<\infty$. Тогда $r_{1}=r_{4}=r_{16}=m$. Любая система образующих пространства $R[\boldsymbol{x}] u$ содержит базис (см. [2]). Следовательно, система образующих $\left(\boldsymbol{x}^{i} u, i \in \Pi\right)$ содержит базис $\left(\boldsymbol{x}^{i} u, i \in \Omega\right)$. Отсюда, $r_{2}=r_{5}=m$. Докажем, что эта система образующих содержит базис специального вида $\left(\boldsymbol{x}^{i} u, i \in \mathscr{F}\right)$, где $\mathscr{F}$ - диаграмма Ферре, и следовательно, $r_{3}=r_{6}=m$. Доказательство уточняет доказательство предложения 1.7(a) в [6] для пространств над полем.

Для наглядности рассмотрим случай $k=2$. Требуемую диаграмму Ферре $\mathscr{F}$ построим по строкам. Расположим все сдвиги 2-ЛРП $u$ в виде таблицы

$\begin{array}{rrrrr}u & x_{1} u & x_{1}^{2} u & x_{1}^{3} u & \ldots \\ x_{2} u & x_{1} x_{2} u & x_{1}^{2} x_{2} u & x_{1}^{3} x_{2} u & \ldots \\ x_{2}^{2} u & x_{1} x_{2}^{2} u & x_{1}^{2} x_{2}^{2} u & x_{1}^{3} x_{2}^{2} u & \ldots \\ x_{2}^{3} u & x_{1} x_{2}^{3} u & x_{1}^{2} x_{2}^{3} u & x_{1}^{3} x_{2}^{3} u & \ldots \\ \ldots \ldots \ldots \ldots \ldots \ldots \ldots \ldots \ldots \ldots \ldots \ldots \ldots\end{array}$

нумерация строк и столбцов в которой начинается с нуля. Двигаясь по нулевой строке таблицы, включим в диаграмму $\mathscr{F}$ точки $(0,0),(1,0), \ldots,\left(m_{0}-1,0\right)$, где $m_{0} \geqslant 0-$ наименьшее число, при котором сдвиг $x_{1}^{m_{0}} u$ линейно выражается через предыдущие сдвиги в нулевой строке. Тогда не только $x_{1}^{m_{0}} u$, но и все сдвиги в нулевой строке линейно выражаются через первые $m_{0}$ сдвигов. Двигаясь далее по первой строке таблицы, включим в диаграмму $\mathscr{F}$ точки $(0,1),(1,1), \ldots,\left(m_{1}-1,1\right)$, где $m_{1}$ - наименьшее неотрицательное число, при котором сдвиг $x_{1}^{m_{1}} x_{2} u$ линейно выражается через первые $m_{0}$ сдвигов в нулевой строке и первые $m_{1}$ сдвигов в первой строке. Тогда $m_{1} \leqslant m_{0}$, причем не только $x_{1}^{m_{1}} x_{2} u$, но и все сдвиги в первой строке линейно выражаются через указанные $m_{0}+m_{1}$ сдвигов. И так далее. Построение будет завершено, когда при некотором $r \geqslant 0$ сдвиг $x_{2}^{r} u$ будет линейно выражаться через $m_{0}+\ldots+m_{r-1}$ сдвигов, находящихся в предыдущих $r$ строках. Тогда

$$
m_{0} \geqslant m_{1} \geqslant \ldots \geqslant m_{r-1}
$$

и не только $x_{2}^{r} u$, но и все сдвиги ЛРП $u$ будут линейно выражаться через

$$
m_{0}+\ldots+m_{r-1}
$$

указанных сдвигов. Построенное множество

$$
\mathscr{F}=\left\{(i, j): 0 \leqslant i<m_{j}, 0 \leqslant j<r\right\}
$$


является диаграммой Ферре, и $\left(\boldsymbol{x}^{i} u, i \in \mathscr{F}\right)$ - система образующих пространства сдвигов $R[\boldsymbol{x}] u$. По построению эта система образующих линейно независима, то есть является базисом. Тем самым доказано, что $r_{3}=r_{6}=m$. Ввиду предложения 3 $r_{10}=m$.

Неравенство $r_{10} \leqslant r_{13}$ доказано в предложении 4(b).

Докажем (b). Так как $R[\boldsymbol{x}] u$ - пространство размерности $m$ и $M$ - пространство размерности $\nu$, мономорфизм $R[\boldsymbol{x}] u \rightarrow M^{t}$ существует тогда и только тогда, когда $m \leqslant t \nu$. При $\nu<\infty$ это равносильно тому, что $t \geqslant] m / \nu\left[\right.$. Следовательно, $\left.r_{7}=\right] m / \nu[$. Случай $\nu=\infty$ тривиален.

Докажем (c). Единственность минимального многочлена следует из предложения 10. Согласно предложению $9 r_{10} \geqslant r_{11}$. Докажем, что $r_{10} \leqslant r_{11}$. Пусть $u$ является выходной последовательностью 1-ЛРС

$$
\langle\chi,\{0,1, \ldots, l-1\}\rangle, \quad \chi \subseteq R[x], \quad l=r_{11} .
$$

Так как $R[x]$ - кольцо главных левых (и главных правых) идеалов, то $R[x] \chi=$ $R[x] F(x)$, где многочлен $F(x)$ можно считать унитарным. Тогда

$$
L_{M}(\chi)=L_{M}(R[x] \chi)=L_{M}(R[x] F)=L_{M}(F)
$$

и $\langle\chi,\{0,1, \ldots, l-1\}\rangle=\langle F(x),\{0,1, \ldots, l-1\}\rangle$ есть ЛРС. Из последнего следует, что $\operatorname{deg} F(x)=l$. А поскольку $u \in L_{M}(\chi)=L_{M}(F)$, то $r_{10} \leqslant \operatorname{deg} F(x)=l$, то есть $r_{10} \leqslant r_{11}$. В итоге $r_{10}=r_{11}$, то есть $r_{11}=m$.

Отметим, что для $k$-ЛРП над полем равенства $r_{1}=r_{2}=r_{3}=r_{4}=r_{5}=r_{6}$ ранее были доказаны в [6].

Из предложения 12(b) и соотношений (1) следует, что

$$
] m / \nu\left[=r_{7} \leqslant r_{8} \leqslant r_{9} .\right.
$$

Согласно примеру 5 неравенства $r_{7} \leqslant r_{8} \leqslant r_{9}$ для 1-ЛРП над векторными пространствами могут обращаться в равенства. Следующий пример показывает, что неравенство $r_{7} \leqslant r_{8}$ может быть строгим даже если $R-$ поле и $k=1$. Пример, когда $m<r_{13}$ для ЛРП над векторным пространством, не найден.

Пример 10. Пусть $R$ - поле, $M=R^{2}$,

$$
u=\left(\left(\begin{array}{l}
0 \\
0
\end{array}\right),\left(\begin{array}{l}
1 \\
0
\end{array}\right),\left(\begin{array}{l}
0 \\
0
\end{array}\right),\left(\begin{array}{l}
0 \\
0
\end{array}\right), \ldots\right) \in M^{\langle 1\rangle} .
$$

Тогда, в обозначениях предложения $12, m=r_{10}=2, \nu=2$, откуда, $r_{7}=1$. Легко увидеть, что $r_{8}=r_{9}=2$, следовательно, $r_{7}<r_{8}$.

Предложение 13. Пусть $R-$ поле, $u \in \mathscr{L}_{R} M^{\langle k\rangle}, \nu=\operatorname{dim}_{R} M, m-$ параметр, введенный в предложении 12. Тогда

(а) $] m / \nu\left[=r_{7} \leqslant r_{8} \leqslant r_{9} \leqslant m\right.$, где при $\nu=\infty$ вместо $] m / \nu[$ нужно брать 0 , если $m=0, u 1$, ecлu $m \geqslant 1$;

(b) $m \leqslant r_{17} \leqslant m \nu$;

(c) $m \leqslant r_{11} \nu$; 
(d) если $R$ - конечное поле, то $r_{18}=m$;

(e) если $R$ - поле u $k=1$, то $r_{13}=r_{15}=m, r_{17}=m \nu$;

(f) если $R$ - поле и $M=R$, то $r_{i}=m, i=1, \ldots, 18$.

Доказательство. Докажем (a). В силу предложения 6(b) над коммутативным кольцом $r_{9} \leqslant r_{3}$, и требуемые соотношения вытекают из предложения 12 .

Докажем (b). Если $u \in L_{M}(I)$, то $R[\boldsymbol{x}] u \subseteq L_{M}(I)$ ввиду коммутативности $R$. Следовательно,

$$
m=\operatorname{dim}_{R} R[\boldsymbol{x}] u \leqslant \operatorname{dim}_{R} L_{M}(I)=r_{1}\left(L_{M}(I)\right),
$$

откуда, $m \leqslant r_{17}$. Докажем, что $r_{17} \leqslant m \nu$.

Пусть $\left(\boldsymbol{x}^{i} u, i \in \mathscr{F}\right)$ - базис пространства ${ }_{R} R[\boldsymbol{x}] u$, где $\mathscr{F}$ - диаграмма Ферре мощности $m$ (такой базис построен в доказательстве предложения 12(a)). Тогда для любого $\boldsymbol{r} \in \Delta \mathscr{F}$ последовательность $\boldsymbol{x}^{\boldsymbol{r}} u$ линейно выражается через $\left(\boldsymbol{x}^{\boldsymbol{i}} u, \boldsymbol{i} \in \mathscr{F}\right)$ :

$$
x^{r} u=\sum_{i \in \mathscr{F}} h_{r i} x^{i} u, \quad h_{r i} \in R .
$$

Положим

$$
H_{r}(x)=\sum_{i \in \mathscr{F}} h_{r i} x^{i}
$$

Тогда $\chi=\left\{H_{\boldsymbol{r}}(\boldsymbol{x}), \boldsymbol{r} \in \Delta \mathscr{F}\right\}-$ полная система $\mathscr{F}$-унитарных многочленов из $\operatorname{An}(u)$ и $u \in L_{M}(\chi)$. Так как, ввиду выбора $\chi$, каждая последовательность $v \in L_{M}(\chi)$ определяется диаграммой значений $v[\mathscr{F}]$, отображение

$$
\sigma: L_{M}(\chi) \rightarrow M^{\mathscr{F}}, \quad v \rightarrow v[\mathscr{F}]
$$

является мономорфизмом и

$$
\operatorname{dim} L_{M}(\chi) \leqslant \operatorname{dim} M^{\mathscr{F}}=|\mathscr{F}| \operatorname{dim} M=m \nu .
$$

Для доказательства неравенства $r_{17} \leqslant m \nu$ остается проверить, что $L_{M}(\chi)$ есть ЛРП-семейство, то есть, что идеал $I=R[\boldsymbol{x}] \chi$ унитарен. Из определения $\chi$ следует, что пространство $R[\boldsymbol{x}] / I$ порождается элементами $\left(\boldsymbol{x}^{i}+I, \boldsymbol{i} \in \mathscr{F}\right)$. Следовательно, $\operatorname{dim} R[\boldsymbol{x}] / I \leqslant|\mathscr{F}|=m$. Тогда для любого $s=1, \ldots, k$ элементы $\boldsymbol{x}_{s}^{i}+I, i=0,1, \ldots, m$, линейно зависимы. Это означает, что $I$ содержит унитарный многочлен $F_{s}\left(x_{s}\right)$ степени не выше $m, s \in\{1, \ldots, k\}$, то есть что $I$ унитарен.

Докажем (c). В силу предложений $12(\mathrm{a})$ и $6(\mathrm{f}) m=r_{16} \leqslant r_{11} \nu$.

Докажем (d). Если $R$ конечно, то $r_{18}=\log _{|R|}|R[\boldsymbol{x}] u|=\operatorname{dim} R[\boldsymbol{x}] u=m$.

Докажем (e). По предложению $11 r_{13}=r_{15}=m$ и $r_{17} \leqslant m \nu$. Докажем, что $r_{17} \geqslant m \nu$. Предположим, что $u \in L_{M}(I)$, где $I$ унитарен, $r_{1}\left(L_{M}(I)\right)=r_{17}$. Так как $R[x]$ - кольцо главных идеалов, то $I=R[x] F(x)$ для некоторого унитарного многочлена $F(x)$. Тогда $u \in L_{M}(I)=L_{M}(F)$, и следовательно, $\operatorname{deg} F(x) \geqslant r_{10}=m$. Отсюда,

$$
r_{17}=r_{1}\left(L_{M}(I)\right)=\operatorname{dim} L_{M}(I)=\operatorname{dim} L_{M}(F)=\operatorname{deg} F(x) \operatorname{dim} M \geqslant m \nu .
$$

Докажем (f). Если $M=R$, то $\nu=1$, и из (a), (b), (d) получаем, что

$$
r_{7}=r_{8}=r_{9}=r_{17}=r_{18}=m \text {. }
$$


Ввиду (7) и предложения $8 r_{12}=r_{13} \leqslant r_{14}=r_{15}$. По предложениям 4(b,c) и $6(\mathrm{~d})$

$$
m=r_{10} \leqslant r_{13}, \quad r_{11} \leqslant r_{15}, \quad r_{11} \geqslant r_{14} .
$$

Следовательно,

$$
m \leqslant r_{12}=r_{13} \leqslant r_{14}=r_{15}=r_{11}
$$

Докажем, что $r_{11} \leqslant m$.

Пусть $\mathscr{F}, \chi, \sigma$ - те же, что и в доказательстве (b). Тогда $u \in L_{M}(\chi)$, и так как $R$ коммутативно, то $R[\boldsymbol{x}] u \subseteq L_{M}(\chi)$. Учитывая, что в нашем случае $M=R$, получаем, что

$$
m=\operatorname{dim} R[\boldsymbol{x}] u \leqslant \operatorname{dim} L_{M}(\chi) \leqslant \operatorname{dim} M^{\mathscr{F}}=|\mathscr{F}|=m .
$$

Следовательно, $\operatorname{dim} L_{M}(\chi)=\operatorname{dim} M^{\mathscr{F}}$, и $\sigma$ является не только мономорфизмом, но и изоморфизмом. Это означает, что пара $\langle\chi, \mathscr{F}\rangle$ есть $k$-ЛРС с выходом $u$, откуда, $r_{11} \leqslant|\mathscr{F}|=m$.

Суммируя сказанное, получим, что $r_{i}=m$ для всех $i=1, \ldots, 18$.

Пример 5 показывает, что неравенства $r_{9} \leqslant m, m \leqslant r_{11} \nu$ в случае 1 -ЛРП над векторным пространством над полем могут быть строгими. Неравенство $r_{9} \leqslant m$, неравенство $m \leqslant r_{11} \nu$ при $\nu=1$, а также оба неравенства $m \leqslant r_{17} \leqslant m \nu$ могут обращаться в равенства, это видно из формулировки предложения $13(\mathrm{e}, \mathrm{f})$.

Доказательство предложения 13 дает способ построения $k$-линейных регистров сдвига и скалярных $\mathscr{F}$-ЛРС систем для $k$-ЛРП над полем. Кратко изложим его здесь отдельно. Пусть $R$ - поле, $u \in \mathscr{L}_{R} R^{\langle k\rangle}$. В доказательстве предложения 12(а) указан способ построения базиса $\left(\boldsymbol{x}^{i} u, \boldsymbol{i} \in \mathscr{F}\right)$ пространства сдвигов $R[\boldsymbol{x}] u$, где $\mathscr{F}$ - диаграмма Ферре мощности $m=\operatorname{dim} R[\boldsymbol{x}] u$. Пусть $\chi=\left\{H_{\boldsymbol{r}}(\boldsymbol{x}), \boldsymbol{r} \in \Delta \mathscr{F}\right\}-$ полная система $\mathscr{F}$-унитарных многочленов из $\operatorname{An}(u)$, определяемых соотношениями $(17),(18)$. Кроме того, определим отображения $\varphi_{1}, \ldots, \varphi_{k}: R^{\mathscr{F}} \rightarrow R^{\mathscr{F}}$ следующим образом:

$$
\varphi_{s}(\delta[\mathscr{F}])=\lambda[\mathscr{F}]
$$

где

$$
\lambda(\boldsymbol{j})= \begin{cases}\delta\left(\boldsymbol{j}+\mathbf{1}_{s}\right), & \text { если } \boldsymbol{j}+\mathbf{1}_{s} \in \mathscr{F}, \\ \sum_{\boldsymbol{i} \in \mathscr{F}} h_{\boldsymbol{r} i} \delta(\boldsymbol{i}), & \text { если } \boldsymbol{j}+\mathbf{1}_{s}=\boldsymbol{r} \in \Delta \mathscr{F},\end{cases}
$$

$\delta[\mathscr{F}], \lambda[\mathscr{F}] \in R^{\mathscr{F}}, j \in \mathscr{F}, s=1, \ldots, k$. Из $(20)$ следует, что $\varphi_{1}, \ldots, \varphi_{k} \in \operatorname{End}\left(R^{\mathscr{F}}\right)$, и что выполняется условие (5) из определения $\mathscr{F}$-ЛРС системы.

Следствие 5. Пусть $R-$ поле, $M=R, u \in \mathscr{L}_{R} R^{\langle k\rangle}$. Тогда при введенных выше обозначениях

(a) пара $\langle\chi, \mathscr{F}\rangle$ есть $k$-ЛРC, въходом которого является $u$;

(b) $\varphi=\left(\varphi_{1}, \ldots, \varphi_{k}\right)$ естъ $\mathscr{F}$-ЛРC система, для которой

$$
u[i+\mathscr{F}]=\varphi^{i}(u[\mathscr{F}]), \quad i \in \mathbf{N}_{0}^{k} .
$$


Доказательство. Утверждение (а) было получено при доказательстве предложения 13(f). $L_{R}(\chi)$

Докажем (b). Из (20) следует, что для произвольной последовательности $v \in$

$$
\varphi_{s}(v[\mathscr{F}])=v\left[\mathbf{1}_{s}+\mathscr{F}\right], \quad s=1, \ldots, k
$$

Следовательно,

$$
\varphi_{s}\left(\varphi_{t}(v[\mathscr{F}])\right)=v\left[\mathbf{1}_{s}+\mathbf{1}_{t}+\mathscr{F}\right]=v\left[\mathbf{1}_{t}+\mathbf{1}_{s}+\mathscr{F}\right]=\varphi_{t}\left(\varphi_{s}(v[\mathscr{F}])\right)
$$

Таким образом, $\varphi_{s}$ и $\varphi_{t}$ перестановочны на диаграммах вида $v[\mathscr{F}], v \in L_{R}(\chi)$. Так как, согласно доказательству предложения 13(f), отображение (19) биективно, то $\varphi_{s}$ и $\varphi_{t}$ перестановочны на всех диаграммах из $R^{\mathscr{F}}$, то есть они перестановочны. Следовательно, $\varphi$ есть $\mathscr{F}$-ЛРС система. При этом из (21) для $v=u$ следует, что

$$
u[i+\mathscr{F}]=\varphi^{i}(u[\mathscr{F}]), \quad i \in \mathbf{N}_{0}^{k}
$$

Линейные регистры сдвига, построенные в следствии 5(a), аналогичны тем, которые рассматривались в работах Сакаты (см., например, [21, 22, 23]).

\section{0. Линейная сложность в расширениях}

Пусть $R \subseteq Q-$ расширение кольца $R, u \in \mathscr{L}_{R} M^{\langle k\rangle},[1 \otimes u]$ - последовательность над модулем $Q \otimes M$, определенная в разделе 3 . Здесь и далее $\otimes=\stackrel{R}{ }$. Отметим, что канонический гомоморфизм $R$-модулей

$$
\alpha: M \rightarrow Q \otimes M, \quad x \rightarrow 1 \otimes x
$$

не всегда является мономорфизмом (например, если $R=\mathbf{Z}, Q=\mathbf{Q}, M=\mathbf{Z}_{2}$ ).

Напомним (см. [5], стр. 46), что правый модуль $Q_{R}$ называется локально свободным, если любое его конечное подмножество содержится в некотором свободном подмодуле модуля $Q_{R}$. Свободный модуль является локально свободным. Правый модуль $Q_{R}$ называется плоским, если для любого мономорфизма $R$-модулей $\varphi: M \rightarrow N$ канонический гомоморфизм $Q$-модулей

$$
1 \otimes \varphi: Q \otimes M \rightarrow Q \otimes N, \quad q \otimes n \rightarrow q \otimes \varphi(n),
$$

является мономорфизмом. Свободный модуль является плоским (см. теорему 10.4.4 в [4]), и в силу следствия 10.4.6 в [4] каждый локально свободный модуль является плоским.

Исследуем модуль сдвигов $Q[\boldsymbol{x}][1 \otimes u]$ последовательности $[1 \otimes u]$, получающейся при расширении $R \subseteq Q$ кольца $R$.

Лемма 2. Пусть $R \subseteq Q$ - расширение колъца $R, u \in M^{\langle k\rangle}$. Тогда отображение

$$
\psi: Q \otimes R[\boldsymbol{x}] u \rightarrow Q[\boldsymbol{x}][1 \otimes u], \quad q \otimes F(\boldsymbol{x}) u \rightarrow q F(\boldsymbol{x})[1 \otimes u],
$$

является эпиморфизмом $Q$-модулей. Если выполняется одно из условий

(a) правый модуль $Q_{R}$ свободен; 
(b) $R$ коммутативно, правый модуль $Q_{R}$ локалъно свободен, $u \in \mathscr{L}_{R} M^{\langle k\rangle}$, то $\psi$ - изоморфизм.

Доказателъство. Рассмотрим отображение

$$
Q \times R[\boldsymbol{x}] u \rightarrow Q[\boldsymbol{x}][1 \otimes u], \quad(q, F(\boldsymbol{x}) u) \rightarrow q F(\boldsymbol{x})[1 \otimes u] .
$$

Это отображение билинейно над $R$, поэтому оно индуцирует гомоморфизм абелевых групп, задаваемый соотношением (23). Очевидно, $\psi-$ гомоморфизм $Q$-модулей. Если

$$
v=H(\boldsymbol{x})[1 \otimes u] \in Q[\boldsymbol{x}][1 \otimes u]
$$

где

$$
H(\boldsymbol{x})=\sum h_{j} \boldsymbol{x}^{\boldsymbol{j}} \in Q[\boldsymbol{x}]
$$

то

$$
v=\psi\left(\sum h_{j} \otimes x^{j} u\right)
$$

Следовательно, $\psi$ - эпиморфизм.

Пусть выполнено условие (а). Пусть $z \in Q \otimes R[\boldsymbol{x}] u, \psi(z)=0$. Элемент $z$ представляется в виде

$$
z=\sum q_{j} \otimes \boldsymbol{x}^{j} u, \quad q_{j} \in Q,
$$

где лишь конечное число $q_{j}$ не равно нулю. Тогда

$$
\psi(z)=\sum q_{j} x^{j}[1 \otimes u] .
$$

Равенство $\psi(z)=0$ означает, что в модуле $Q \otimes M$ выполняются равенства

$$
\sum_{j \in \mathbf{N}_{\mathbf{0}}^{k}} q_{j} \otimes \boldsymbol{x}^{j} u(i)=0, \quad i \in \mathbf{N}_{0}^{k} .
$$

Пусть $\left(\alpha_{l}, l \in L\right)$ - базис свободного правого модуля $Q_{R}$. Запишем элементы $q_{j}$ в виде

$$
q_{j}=\sum_{l \in L} \alpha_{l} r_{j l}, \quad r_{j l} \in R,
$$

где для каждого $j$ лишь конечное число элементов $r_{j l}$ не равно 0 . Тогда согласно (24) для каждого $i \in \mathbf{N}_{0}^{k}$

$$
\sum_{j \in \mathbf{N}_{0}^{k}} \sum_{l \in L} \alpha_{l} r_{j l} \otimes \boldsymbol{x}^{j} u(i)=\sum_{l \in L} \alpha_{l} \otimes w_{l}(\boldsymbol{i})=0
$$

в модуле $Q \otimes M$, где

$$
w_{l}=\sum_{j \in \mathbf{N}_{0}^{k}} r_{j l} \boldsymbol{x}^{j} u \in R[\boldsymbol{x}] u, \quad l \in L
$$

В силу свойства перестановочности прямых сумм и тензорных произведений (см. предлюжение 10.2.3 в [4]),

$$
Q \otimes M=\left(\bigoplus \alpha_{l} R\right) \otimes M=\bigoplus\left(\alpha_{l} R \otimes M\right),
$$


причем $\alpha_{l} R \otimes M \cong R \otimes M \cong M$. Поэтому из (26) следует, что $w_{l}(i)=0, l \in L, i \in \mathbf{N}_{0}^{k}$, то есть $w_{l}=0, l \in L$. Теперь

$$
z=\sum_{j \in \mathbf{N}_{0}^{k}} q_{j} \otimes \boldsymbol{x}^{j} u=\sum_{j \in \mathbf{N}_{0}^{k}} \sum_{l \in L} \alpha_{l} r_{j l} \otimes \boldsymbol{x}^{j} u=\sum_{l \in L} \alpha_{l} \otimes w_{l}=0 .
$$

Следовательно, $\psi$ - мономорфизм.

Пусть выполнено условие (b). Как и в случае (a), пусть $z \in Q \otimes R[\boldsymbol{x}] u, \psi(z)=0$, то есть для любого $i \in \mathbf{N}_{0}^{k}$ выполняется соотношение (24). Пусть $\Pi-$ начальный параллелепипед $k$-ЛРП $u$. Тогда, ввиду (24),

$$
\sum_{j \in \mathrm{N}_{0}^{k}} q_{j} \otimes \boldsymbol{x}^{j} u(i)=0, \quad i \in \Pi,
$$

в модуле $Q \otimes M$. Стандартные рассуждения (см., например, лемму 6.2 на стр. 47 в [5], доказательство предложения 10.4 .5 на стр. 255 в [4]) показывают, что конечное число равенств (29) выполняется также в модуле $Q^{\prime} \otimes M$, где $Q^{\prime}$ - конечно порожденный подмодуль правого модуля $Q_{R}$, содержащий все элементы $q_{j}$ ( $Q^{\prime}$ порождается элементами $q_{j}$ и элементами из $Q$, участвующими в выводе всех $|\Pi|$ равенств (29) с использованием определения тензорного произведения $Q \otimes M)$. Так как $Q_{R}$ локально свободен, то $Q^{\prime} \subseteq Q^{\prime \prime}$, где $Q_{R}^{\prime \prime}$ свободный. Равенства (29) выполняются также в $Q^{\prime \prime} \otimes M$. Пусть $\left(\alpha_{l}, l \in L\right)-$ базис $Q_{R}^{\prime \prime}$. Представим элементы $q_{j}$ в виде (25). Тогда при всех $i \in \Pi$ в модуле $Q^{\prime \prime} \otimes M$ выполняются равенства (26), в которых последовательности $w_{l}$ определены в (27). Отсюда получаем, что $w_{l}(\boldsymbol{i})=0, l \in L, \boldsymbol{i} \in \Pi$, то есть $w_{l}[\Pi]=0, l \in L$. Так как $w_{l} \in R[\boldsymbol{x}] u$ и $R$ коммутативно, то П является начальным параллелепипедом $k$-ЛРП $w_{l}$. Следовательно, $w_{l}=0, l \in L$. Теперь соотношения (28) показывают, что $z=0$. Поэтому $\psi-$ мономорфизм.

В дальнейшем мы будем использовать сокращенные обозначения

$$
r_{i}=r_{i}(u), \quad r_{i}^{Q}=r_{i}^{Q}(1 \otimes u) .
$$

Предложение 14. Пустъ $R \subseteq Q, u \in \mathscr{L}_{R} M^{\langle k\rangle}$. Тогда

$$
\operatorname{An}_{R[x]}(u) \subseteq \operatorname{An}_{Q[x]}([1 \otimes u])
$$

$u[1 \otimes u]-k$-ЛРП над $Q$-модулем $Q \otimes M$ с теми же элементарными характеристическими многочленами, что и $k$-ЛРП и. Справедливы следующие свойства.

(a) $r_{i} \geqslant r_{i}^{Q}, i=1,2,3,5,6,10,16$.

(b) Если отображение (22) является мономорфизмом, то $r_{8} \leqslant r_{8}^{Q}, r_{9} \leqslant r_{9}^{Q}$.

(c) Если правый модулъ $Q_{R}$ локалъно свободен, то $r_{8} \geqslant r_{8}^{Q}, r_{9} \geqslant r_{9}^{Q}$.

(d) Если $R$ коммутативно и правый модулъ $Q_{R}$ локалъно свободен, то

$$
r_{4} \leqslant r_{4}^{Q}, \quad r_{5}=r_{5}^{Q}, \quad r_{6}=r_{6}^{Q}, \quad r_{7} \geqslant r_{7}^{Q}
$$

(е) Если правъй модуль $Q_{R}$ свободен, то

$$
r_{4} \leqslant r_{4}^{Q}, \quad r_{5}=r_{5}^{Q}, \quad r_{6}=r_{6}^{Q}, \quad r_{7} \geqslant r_{7}^{Q} .
$$


Доказательство. Пусть

$$
F(x)=\sum f_{j} x^{j} \in \operatorname{An}_{R[x]}(u), \quad v=F(x)[1 \otimes u] .
$$

Тогда для любого $i \in \mathbf{N}_{0}^{k}$

$$
\begin{aligned}
v(\boldsymbol{i}) & =\sum_{\boldsymbol{j} \in \mathbf{N}_{\mathbf{0}}^{k}}\left(f_{\boldsymbol{j}} \boldsymbol{x}^{\boldsymbol{j}}[1 \otimes u]\right)(\boldsymbol{i})=\sum_{\boldsymbol{j} \in \mathbf{N}_{\mathbf{0}}^{k}} f_{\boldsymbol{j}} \otimes u(\boldsymbol{i}+\boldsymbol{j}) \\
& =1 \otimes \sum_{\boldsymbol{j} \in \mathbf{N}_{\mathbf{0}}^{k}} f_{\boldsymbol{j}} u(\boldsymbol{i}+\boldsymbol{j})=1 \otimes 0=0
\end{aligned}
$$

то есть $v=0$. Это доказывает первую часть предложения.

Докажем теперь утверждение (a). Если $R$-модуль $R[\boldsymbol{x}] u$ порождается последовательностями $v_{1}, \ldots, v_{n}$, то $Q$-модуль $Q[\boldsymbol{x}][1 \otimes u]$ порождается последовательностями $\left[1 \otimes v_{1}\right], \ldots,\left[1 \otimes v_{n}\right]$. Отсюда следует, что $r_{1} \geqslant r_{1}^{Q}$. Неравенства $r_{2} \geqslant r_{2}^{Q}$ и $r_{3} \geqslant r_{3}^{Q}$ проверяются аналогично. Ввиду предложения $3 r_{10} \geqslant r_{10}^{Q}$.

Если последовательности $\left(\boldsymbol{x}^{i}[1 \otimes u], i \in \Omega\right)$ линейно независимы над $Q$, то последовательности $\left(\boldsymbol{x}^{i} u, i \in \Omega\right)$ линейно независимы над' $R$ (предположить противное). Следовательно, $r_{5} \geqslant r_{5}^{Q}$. Аналогично, $r_{6} \geqslant r_{6}^{Q}$.

Если $R[\boldsymbol{x}] u \subseteq R\left(v_{1}, \ldots, v_{n}\right)$, то

$$
Q[\boldsymbol{x}][1 \otimes u] \subseteq Q\left(\left[1 \otimes v_{1}\right], \ldots,\left[1 \otimes v_{n}\right]\right) .
$$

Следовательно, $r_{16} \geqslant r_{16}^{Q}$.

Докажем (b). Пусть $\Omega$ - определяющее множество для последовательности $[1 \otimes u]$ в смысле определения $r_{8}^{Q}$. Докажем, что $\Omega$ - определяющее множество для последовательности $u$ в смысле определения $r_{8}$. Пусть $v \in R[\boldsymbol{x}] u, v[\Omega]=0$. Тогда $[1 \otimes v] \in Q[\boldsymbol{x}][1 \otimes u]$ и

$$
[1 \otimes v](i)=1 \otimes v(i)=1 \otimes 0=0, \quad i \in \Omega,
$$

то есть $[1 \otimes v][\Omega]=0$. Следовательно, $[1 \otimes v]=0$, то есть

$$
1 \otimes v(i)=0, \quad i \in \mathbf{N}_{0}^{k}
$$

Так как отображение (22) является мономорфизмом, то $v(i)=0, i \in \mathbf{N}_{0}^{k}$, то есть $v=0$. Этим доказано, что $\Omega$ - определяющее множество для последовательности $u$ в смысле определения $r_{8}$. Отсюда следует, что $r_{8} \leqslant r_{8}^{Q}$. Доказательство неравенства $r_{9} \leqslant r_{9}^{Q}$ получается заменой $\Omega$ на $\mathscr{F}$.

Докажем (c). Пусть $\Omega$ - определяющее множество для последовательности $u$ в смысле определения $r_{8}$. Докажем, что $\Omega$ - определяющее множество для последовательности $[1 \otimes u]$ в смысле определения $r_{8}^{Q}$. Пусть

$$
v \in Q[\boldsymbol{x}][1 \otimes u], \quad v[\Omega]=0 .
$$

Тогда $v$ представляется в виде

$$
v=\sum q_{j} x^{j}[1 \otimes u], \quad q_{j} \in Q
$$


и

$$
v(i)=\sum_{j \in \mathbf{N}_{0}^{k}} q_{j} \otimes \boldsymbol{x}^{j} u(i)=0, \quad i \in \Omega
$$

в модуле $Q \otimes M$. Будем рассуждать, как в доказательстве леммы 2. Равенства (30) выполняются также в модуле $Q^{\prime \prime} \otimes M$, где $Q^{\prime \prime}-$ свободный подмодуль правого модуля $Q_{R}$. Пусть $\left(\alpha_{l}, l \in L\right)-$ базис $Q_{R}^{\prime \prime}$. Запишем элементы $q_{j}$ в виде (25). Тогда для всех $i \in \Omega$ в модуле $Q^{\prime \prime} \otimes M$ выполняются равенства (26), где $w_{l} \in R[\boldsymbol{x}] u$ определены в (27). Из (26) следует, что $w_{l}(i)=0, l \in L, i \in \Omega$, то есть $w_{l}[\Omega]=0, l \in L$. Так как $\Omega$ - определяющее множество для ЛРП $u$ в смысле определения $r_{8}$, то $w_{l}=0, l \in L$. Теперь для любого $i \in \mathbf{N}_{0}^{k}$

$$
v(\boldsymbol{i})=\sum_{\boldsymbol{j} \in \mathbf{N}_{\mathbf{0}}^{k}} q_{\boldsymbol{j}} \otimes \boldsymbol{x}^{\boldsymbol{j}} u(\boldsymbol{i})=\sum_{\boldsymbol{j} \in \mathbf{N}_{\mathbf{0}}^{k}} \sum_{l \in L} \alpha_{l} r_{\boldsymbol{j} l} \otimes \boldsymbol{x}^{\boldsymbol{j}} u(\boldsymbol{i})=\sum_{l \in L} \alpha_{l} \otimes w_{l}(\boldsymbol{i})=0
$$

в модулях $Q^{\prime \prime} \otimes M$ и $Q \otimes M$. Следовательно, $v=0$. Из доказанного вытекает неравенство $r_{8} \geqslant r_{8}^{Q}$. Доказательство того, что $r_{9} \geqslant r_{9}^{Q}$, получается заменой $\Omega$ на $\mathscr{F}$.

Докажем (d) и (f). Докажем неравенства $r_{i} \leqslant r_{i}^{Q}, i=4,5,6$. Пусть последовательности $v_{1}, \ldots, v_{n} \in R[\boldsymbol{x}] u$ линейно независимы над $R$. Тогда последовательности $\left[1 \otimes v_{1}\right], \ldots,\left[1 \otimes v_{n}\right]$ принадлежат $Q[\boldsymbol{x}][1 \otimes u]$. Докажем, что они линейно независимы над $Q$. Предположим, что

$$
\sum q_{j}\left[1 \otimes v_{j}\right]=0, \quad q_{j} \in Q, \quad j=1, \ldots, n .
$$

Рассматривая прообраз этой последовательности при действии изоморфизма $\psi$ из леммы 2 , получим, что

$$
\sum q_{j} \otimes v_{j}=0
$$

в модуле $Q \otimes R[\boldsymbol{x}] u$. Отсюда, в силу лемма 6.2 на стр. 47 в $[5], q_{j}=0, j=1, \ldots, n$, что и требовалось. Из доказанного следует, что $r_{4} \leqslant r_{4}^{Q}$.

Аналогично доказываются неравенства $r_{5} \leqslant r_{5}^{Q}, r_{6} \leqslant r_{6}^{Q}$. С учетом (а) получаем, что $r_{5}=r_{5}^{Q}, r_{6}=r_{6}^{Q}$.

Докажем неравенство $r_{7} \geqslant r_{7}^{Q}$. Пусть $\varphi: R[\boldsymbol{x}] u \rightarrow M^{t}$ - мономорфизм $R$-модулей. Рассмотрим композицию $Q$-гомоморфизмов

$$
\varphi_{1}: Q[\boldsymbol{x}][1 \otimes u] \stackrel{\psi^{-1}}{\longrightarrow} Q \otimes R[\boldsymbol{x}] u \stackrel{1 \otimes \varphi}{\longrightarrow} Q \otimes M^{t} \stackrel{\alpha}{\longrightarrow}(Q \otimes M)^{t}
$$

где $\psi$ - изоморфизм из леммы $2,1 \otimes \varphi-$ канонический гомоморфизм, $\alpha-$ канонический изоморфизм. Так как правый модуль $Q_{R}$ локально свободен, он является плоским, и $1 \otimes \varphi-$ мономорфизм. Следовательно, $\varphi_{1}-$ мономорфизм $Q$-модулей. Отсюда следует, что $r_{7} \geqslant r_{7}^{Q}$.

Отметим, что в предложении $14(\mathrm{a})$ отсутствует неравенство $r_{4} \geqslant r_{4}^{Q}$. Его не удалось ни доказать, ни опровергнуть примером. Это неравенство верно в случае, когда $R$ - левая область Оре и $Q$ - ее левое тело частных (см. предложение 16(a)). Следующие примеры показывают, что неравенства $r_{i} \geqslant r_{i}^{Q}, i=1,2,3,7,8,9,16$, доказанные в различных пунктах предложения 14, могут быть строгими. 
Пример 11. Пусть $R=\mathbf{Z}, M=Z_{\mathbf{4}}, u=(2,2,2, \ldots) \in M^{\langle 1\rangle}$, и $\mathbf{Z} \subset \mathbf{Q}$ - pacширение кольца $\mathbf{Z}$. Тогда соответствующее расширение модуля $Z_{4}$ равно $\mathbf{Q} \otimes \mathbf{Z} Z_{4}=0$. Следовательно, $[1 \otimes u]=0$. В этом примере

$$
r_{1}=r_{2}=r_{3}=r_{7}=r_{8}=r_{9}=r_{10}=r_{16}=1, \quad r_{4}=r_{5}=r_{6}=0,
$$

и $r_{i}^{Q}=0, i=1, \ldots, 17$.

Рассмотрим более содержательный пример 1-ЛРП над коммутативной областью целостности $R$.

Пример 12. Пусть $P$ - поле, $\alpha, \beta$ - свободные коммутирующие переменные,

$$
R=P[\alpha, \beta] /\left(\alpha^{2}-\beta^{3}\right) .
$$

Так как многочлен $x^{2}-y^{3} \in P[x, y]$ неприводим, порождаемый им идеал в кольце $P[x, y]$ является простым. Отсюда следует, что $R$ - коммутативное кольцо без делителей нуля. Рассмотрим последовательность

$$
u=\left(\beta, \alpha, \beta^{2}, \alpha \beta, \beta^{3}, \alpha \beta^{2}, \ldots, \beta^{i+1}, \alpha \beta^{i}, \ldots\right) \in R^{\langle 1\rangle} .
$$

Легко видеть, что $u$ есть ЛРП с минимальным многочленом $F(x)=x^{2}-\beta$. Поэтому $r_{10}=2$. В силу следствия 3

$$
r_{i}=\operatorname{rank} u=2, \quad i=1,2,3,10, \ldots, 15,
$$

а также $r_{16}, r_{17} \leqslant 2$.

Пусть $Q$ - поле частных кольца $R$. Тогда $u-$ ЛРП над $Q$ с минимальным многочленом $x-\alpha / \beta$. Следовательно, $r_{10}^{Q}=1$. По предложению $13(\mathrm{e})$

$$
r_{i}^{Q}=1, \quad i=1, \ldots, 17 .
$$

Так как $M=R$, отображение (22) является мономорфизмом. Поле частных $Q$ всегда является локально свободным правым $R$-модулем (см. [5], стр.47). Следовательно, по предложению $14(\mathrm{~b}, \mathrm{~d})$,

$$
r_{i} \leqslant r_{i}^{Q}, \quad i=4,5,6,8,9
$$

Отсюда,

$$
r_{4}=r_{5}=r_{6}=r_{8}=r_{9}=1 \text {. }
$$

Так как $r_{7} \leqslant r_{8}$ в силу (1), то $r_{7}=1$.

В последнем примере $r_{i}>r_{i}^{Q}, i=1,2,3,10, \ldots, 15$. Кроме того, этот пример является иллюстрацией к предложению 9.

Рассмотрим теперь расширения тел и полей.

Предложение 15. Пусть $R \subseteq Q-$ расширение тел,

$$
u \in \mathscr{L}_{R} M^{\langle k\rangle}, \quad \nu=r_{1}(M)=\operatorname{dim}_{R} M, \quad m=r_{1}(u) .
$$

Тогда

$$
\left.r_{i}=r_{i}^{Q}=m, \quad i=1, \ldots, 6,10,16, \quad\right] m / \nu\left[=r_{7}=r_{7}^{Q} \leqslant r_{8}=r_{8}^{Q} \leqslant r_{9}=r_{9}^{Q},\right.
$$

где при $\nu=\infty$ вместо $] m / \nu[$ нужно брать 0 , если $m=0, u 1$, если $m \geqslant 1$. Справедливи следующие утверждения. 
(a) Eсли $k=1$, то минимальный многочлен ЛРП $и$ над телом $R$ совпадает $c$ минимальньм многочленом ЛРП $[1 \otimes u]$ над телом $Q, u r_{11}=r_{11}^{Q}=m$.

(b) Если $R-$ поле, то $r_{9} \leqslant m$.

(c) Если $R \subseteq Q-$ расширение полей $u k=1$, то

$$
r_{13}=r_{13}^{Q}=r_{15}=r_{15}^{Q}=m, \quad r_{17}=r_{17}^{Q}=m \nu
$$

(d) Если $R \subseteq Q-$ расширение полей и $M=R$, то

$$
r_{i}=r_{i}^{Q}=m, \quad i=1, \ldots, 18
$$

(е) Если $R \subseteq Q-$ расширение конечных полей, то $r_{18}=r_{18}^{Q}=m$.

Доказательство. По предложению 12 (a) $r_{i}=m, i=1, \ldots, 6,10,16$. По этому же предложению параметры $r_{i}^{Q},=1, \ldots, 6,10,16$, равны друг другу. Так как модуль $Q_{R}$ свободный, по предложению $14(\mathrm{e}) r_{5}=r_{5}^{Q}$. Следовательно, $r_{i}=r_{i}^{Q}=m, i=$ $1, \ldots, 6,10,16$.

Далее, по предложению 12 (b) $\left.r_{7}=\right] m / \nu\left[, r_{7}^{Q}=\right] m / \nu^{Q}\left[\right.$, где $\nu^{Q}=\operatorname{dim}_{Q} Q \otimes M$. Так как $M=R^{\nu}-$ пространство размерности $\nu$ над $R$, то

$$
Q \otimes M=Q \otimes R^{\nu}=Q^{\nu}
$$

- пространство размерности $\nu$ над $Q$. Следовательно, $\nu=\nu^{Q}$ и $\left.r_{7}=r_{7}^{Q}=\right] m / \nu[$. В силу (1) $r_{7} \leqslant r_{8} \leqslant r_{9}$. Так как модуль ${ }_{R} M$ свободный, он является плоским, поэтому гомоморфизм (22) является мономорфизмом. В силу предложения $14(\mathrm{~b}, \mathrm{c}) r_{8}=r_{8}^{Q}$, $r_{9}=r_{9}^{Q}$. Первая часть предложения доказана.

Докажем (а). По предложению $12(\mathrm{c}) r_{11}=r_{1}$ и $r_{11}^{Q}=r_{1}^{Q}$, откуда в силу уже доказанного $r_{11}=r_{11}^{Q}=m$. По этому же предложению минимальные многочлены последовательностей $u$ и $[1 \otimes u]$ определены однозначно, а по предложению 14 минимальный многочлен ЛРП $u$ является характеристическим многочленом ЛРП $[1 \otimes u]$. Так как $r_{10}=r_{10}^{Q}$, он является минимальным многочленом ЛРП $[1 \otimes u]$.

Утверждение (b) следует из предложения 13(a).

Докажем (c). Так как $r_{1}=r_{1}^{Q}=m$, в силу предложения $13(\mathrm{e}) r_{13}=r_{15}=m$, $r_{17}=m \nu$, а также $r_{13}^{Q}=r_{15}^{Q}=m, r_{17}^{Q}=m \nu^{Q}$. При этом $\nu=\nu^{Q}$, как показано выше.

Докажем (d). По предложению 13(f)

$$
r_{i}=r_{1}, \quad r_{i}^{Q}=r_{1}^{Q}, \quad i=1, \ldots, 18
$$

Остается заметить, что $r_{1}=r_{1}^{Q}$ по доказанному.

Докажем (e). Пользуясь предложением 13(d) и уже доказанным, получаем, что

$$
r_{18}=r_{1}=r_{1}^{Q}=r_{18}^{Q} .
$$




\section{1. Линейная сложность над областями Оре и Безу}

Напомним, что кольцо $R$ называется областью целостности, если оно не содержит левых и правых делителей нуля. Область целостности $R$ называется левой областью Оре, если выполняется левое условие Оре

$$
\forall a, b \in R \backslash 0 \quad R a \cap R b \neq 0,
$$

то есть любые два ненулевые главные левые идеалы имеют ненулевое пересечение. Любая нетерова слева область целостности является левой областью Оре (см. [5], стр. 47). Всякая коммутативная область целостности, очевидно, удовлетворяет левому условию Оре.

Основное свойство левой области Оре $R$ заключается в том, что она вложима в тело, называемое левым телом частных кольца $R$. Это тело строится следующим образом (см. [5], стр.39, [12], стр. 163). На множестве пар $(a, b), a \in R, b \in R \backslash 0$, определим отношение эквивалентности $\sim$, полагая

$$
(a, b) \sim(c, d) \Longleftrightarrow \exists u, v \in R \backslash 0 \quad v a=u c, \quad v b=u d \neq 0 .
$$

Классы эквивалентности будем называть дробями и записывать их в виде

$$
\frac{a}{b}=a / b=b^{-1} a \text {. }
$$

Для любого $s \in R \backslash 0$ верно равенство

$$
\frac{a}{b}=\frac{s a}{s b}
$$

(в (32) положить $u=1, v=s$ ). На множестве $Q$ всех дробей вводятся операции сложения и умножения. Любые две дроби $a / b, c / d \in Q$ можно привести к общему знаменателю: в силу (31)

$$
\exists s, t \in R \backslash 0 \quad s b=t d=w \neq 0,
$$

тогда

и мы полагаем

$$
\frac{a}{b}=\frac{s a}{s b}=\frac{s a}{w}, \quad \frac{c}{d}=\frac{t c}{t d}=\frac{t c}{w}
$$

$$
\frac{a}{b}+\frac{c}{d}=\frac{s a+t c}{w}
$$

Кроме того, из (31) следует, что

$$
\exists u, v \in R, u \neq 0 \quad u a=v d,
$$

и мы полагаем

$$
\frac{a}{b} \frac{c}{d}=\frac{v c}{u b} .
$$

Следующая цепочка равенств в $Q$ показывает естественность такого определения:

$$
u b \frac{a}{b} \frac{c}{d}=u b b^{-1} a d^{-1} c=u a d^{-1} c=v d d^{-1} c=v c .
$$


Отметим, что

$$
\frac{1}{b} \frac{c}{d}=\frac{c}{d b}
$$

(в (33) положить $u=d, v=1$ ) и

$$
\frac{a}{b} \frac{c}{1}=\frac{a c}{b}
$$

(в (33) положить $u=1, v=a$ ). Введенные операции определены корректно (их результат не зависит от представителей классов эквивалентности и от выбора элементов $s, t, u, v)$, и $(Q,+, \cdot)$ есть тело. Отображение $R \rightarrow Q, a \rightarrow a / 1$, является мономорфизмом. Мы отождествляем элементы $a \in R$ и $a / 1 \in Q$ и считаем, что $R \subseteq Q$. При этом соглашении в кольце $Q$ верно равенство $a / b=b^{-1} a$. Левое тело частных $Q$ является локально свободным правым $R$-модулем (см. [5], стр. 47-48).

Отметим, что любое конечное число дробей в кольце $Q$ можно привести к общему знаменателю:

$$
\forall \frac{a_{1}}{b_{1}}, \ldots, \frac{a_{n}}{b_{n}} \in Q \quad \exists c_{1}, \ldots, c_{n} \in R \quad \exists b \in R \backslash 0 \quad \frac{a_{i}}{b_{i}}=\frac{c_{i}}{b}, \quad i=1, \ldots, n .
$$

При $n=2$ это проверено выше. Если $n$ дробей уже приведено к общему знаменателю и $a_{n+1} / b_{n+1} \in Q$, то в силу (31) существуют $s, t \in R \backslash 0$ такие, что $s b=t b_{n+1}=w \neq 0$. Тогда

$$
\frac{a_{i}}{b_{i}}=\frac{c_{i}}{b}=\frac{s c_{i}}{s b}=\frac{s c_{i}}{w}, \quad i=1, \ldots, n, \quad \frac{a_{n+1}}{b_{n+1}}=\frac{t a_{n+1}}{t b_{n+1}}=\frac{t a_{n+1}}{w},
$$

и $n+1$ дробей приведено к общему знаменателю.

Если $M$ - левый модуль над левой областью Oре $R$ с левым телом частных $Q$, то $Q$-модуль $Q \stackrel{R}{\otimes} M$ называется левым модулем частных модуля $M$. Канонический гомоморфизм $R$-модулей, определенный в $(22)$, в данной ситуации имеет ядро, совпадающее с периодической частью модуля $M$ :

$$
\operatorname{Ker} \alpha=t(M)=\{x \in M: \exists a \in R \backslash 0 a x=0\} .
$$

В частности, $\alpha$ - мономорфизм тогда и только тогда, когда $t(M)=0$, то есть $R M$ - модуль без кручения (см. [5], следствие на стр. 46).

Лемма 3. Пусть ${ }_{R} M-$ модуль без кручения над левой областъю Оре $R$ с левым телом частных $Q, u \in M^{\langle k\rangle}$. Тогда эпиморфизм $\psi$ из леммы 2 является изоморфизмом.

Доказательство. Пусть $z \in Q \otimes R[\boldsymbol{x}] u, \psi(z)=0$. Элемент $z$ представляется в виде

$$
z=\sum q_{j} \otimes \boldsymbol{x}^{j} u, \quad q_{j} \in Q
$$

где лишь конечное число $q_{j} \neq 0$. Тогда в модуле $Q \otimes M$ выполняются равенства (24). Приведем дроби $q_{j}, \boldsymbol{j} \in \mathbf{N}_{0}^{k}$, к общему знаменателю:

$$
q_{j}=\frac{a_{j}}{b}, \quad a_{j} \in R, \quad b \in R \backslash 0 .
$$

Тогда соотношение (24) запишется в виде

$$
\frac{1}{b} \sum_{j \in \mathbf{N}_{0}^{k}} a_{j} \otimes \boldsymbol{x}^{j} u(i)=0, \quad i \in \mathbf{N}_{0}^{k}
$$


Умножив слева на $b$, получим, что

$$
\sum_{j \in \mathbf{N}_{0}^{k}} a_{j} \otimes \boldsymbol{x}^{j} u(i)=1 \otimes \sum_{j \in \mathbf{N}_{0}^{k}} a_{j} \boldsymbol{x}^{j} u(i)=0, \quad i \in \mathbf{N}_{0}^{k} .
$$

Так как ${ }_{R} M$ - модуль без кручения, гомоморфизм $\alpha$, определенный в $(22)$, является мономорфизмом. Следовательно,

$$
\sum_{j \in \mathbf{N}_{0}^{k}} a_{j} \boldsymbol{x}^{j} u(i)=0, \quad i \in \mathbf{N}_{0}^{k}
$$

то есть

$$
\sum_{j \in N_{0}^{k}} a_{j} \boldsymbol{x}^{j} u=0
$$

Отсюда,

$$
z=\sum_{j \in \mathbf{N}_{\mathbf{0}}^{k}} q_{j} \otimes \boldsymbol{x}^{j} u=\frac{1}{b} \sum_{j \in \mathbf{N}_{\mathbf{0}}^{k}} a_{j} \otimes \boldsymbol{x}^{j} u=\frac{1}{b} \otimes \sum_{j \in \mathbf{N}_{\mathbf{0}}^{k}} a_{j} \boldsymbol{x}^{j} u=0 .
$$

Тем самым показано, что $\psi-$ мономорфизм.

Предложение 16. Пусть ${ }_{R} M-$ модуль над левой областъю Оре $R$ с левъм телом частных $Q, u \in \mathscr{L}_{R} M^{\langle k\rangle}$. Пусть

$$
m=\operatorname{dim}_{Q} Q[\boldsymbol{x}][1 \otimes u], \quad \nu=r_{1}\left({ }_{R} M\right), \quad \nu^{Q}=r_{1}\left({ }_{Q} Q \otimes M\right)=\operatorname{dim}_{Q} Q \otimes M .
$$

Тогда $m<\infty, \nu^{Q}=r_{4}\left({ }_{R} M\right) \leqslant r_{1}\left({ }_{R} M\right)=\nu u$

$$
m=r_{i}^{Q}, \quad i=1, \ldots, 6,10,16 .
$$

Справедливы следующие утверждения.

(a) $r_{i} \geqslant m, i=1, \ldots, 6,10,16$.

(b) $\left.r_{8} \geqslant r_{8}^{Q}, r_{9} \geqslant r_{9}^{Q},\right] m / \nu^{Q}\left[=r_{7}^{Q} \leqslant r_{8}^{Q} \leqslant r_{9}^{Q}\right.$, где при $\nu=\infty$ вместо $] m / \nu[$ нужно брать 0 , если $m=0, u 1$, если $m \geqslant 1$.

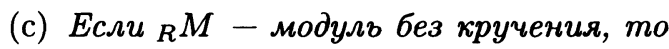

$$
r_{4}=r_{5}=r_{6}=m, \quad r_{7} \geqslant r_{7}^{Q}, \quad r_{8}=r_{8}^{Q}, \quad r_{9}=r_{9}^{Q}
$$

Доказатељьство. Ввиду предложения $14[1 \otimes u]$ есть $k$-ЛРП над $Q$-модулем $Q \otimes M$. Неравенство $m<\infty$ и равенства $r_{i}^{Q}=m, i=1, \ldots, 6,10,16$, доказаны в предложении 12(a). В силу [5] (см. стр. 47-48) наибольшее число линейно независимых элементов произвольного модуля ${ }_{R} M$ равно размерности векторного пространства $Q \otimes M$ над телом $Q$ (где $R$ - левая область Oре, а $Q$ - ее левое тело частных). В наших обозначениях это значит, что $r_{4}\left({ }_{R} M\right)=\nu^{Q}$. Если $R$-модуль $M$ порождается элементами $x_{1}, \ldots, x_{n}$, то $Q$-модуль $Q \otimes M$ порождается элементами $1 \otimes x_{1}, \ldots, 1 \otimes x_{n}$. Следовательно, $r_{1}\left({ }_{Q} Q \otimes M\right) \leqslant r_{1}\left({ }_{R} M\right)$, то есть $\nu^{Q} \leqslant \nu$.

Докажем (а). По предложению 14 (a) $r_{i} \geqslant m, i=1,2,3,5,6,10,16$. Остается доказать, что $r_{4} \geqslant m$. В силу леммы 2 векторное пространство $Q[\boldsymbol{x}][1 \otimes u]$ есть эпиморфный образ пространства $Q \otimes R[\boldsymbol{x}] u$. Следовательно,

$$
\operatorname{dim}_{Q} Q[\boldsymbol{x}][1 \otimes u] \leqslant \operatorname{dim}_{Q} Q \otimes R[\boldsymbol{x}] u
$$


то есть $m \leqslant \operatorname{dim}_{Q} Q \otimes R[\boldsymbol{x}] u$. В силу [5] (см. стр. 47-48)

$$
\operatorname{dim}_{Q} Q \otimes R[\boldsymbol{x}] u=r_{4}\left({ }_{R} R[\boldsymbol{x}] u\right) .
$$

В итоге $m \leqslant r_{4}\left({ }_{R} R[\boldsymbol{x}] u\right)=r_{4}$.

Докажем (b). Поскольку правый модуль $Q_{R}$ локально свободен, по предложению 14(c)

$$
r_{8} \geqslant r_{8}^{Q}, \quad r_{9} \geqslant r_{9}^{Q} .
$$

По предложению $\left.12(\mathrm{~b}) r_{7}^{Q}=\right] m / \nu^{Q}\left[\right.$. Неравенства $r_{7}^{Q} \leqslant r_{8}^{Q} \leqslant r_{9}^{Q}$ взяты из (1).

Докажем (c). По лемме 3 векторные пространства $Q[\boldsymbol{x}][1 \otimes u]$ и $Q \otimes R[\boldsymbol{x}] u$ изоморфны. Как и в доказательстве (a),

$$
r_{4}=r_{4}(R[\boldsymbol{x}] u)=\operatorname{dim}_{Q} Q \otimes R[\boldsymbol{x}] u=\operatorname{dim}_{Q} Q[\boldsymbol{x}][1 \otimes u]=r_{4}^{Q}=m .
$$

В силу (1) $r_{4} \geqslant r_{5} \geqslant r_{6}$ и в силу (а) $r_{6} \geqslant m$. Следовательно, $r_{4}=r_{5}=r_{6}=m$.

Неравенство $r_{7} \geqslant r_{7}^{Q}$ доказывается точно так же, как и в доказательстве предложения $14(\mathrm{~d}, \mathrm{e})$, только вместо леммы 2 нужно использовать лемму 3 . Так как ${ }_{R} M-$ модуль без кручения, гомоморфизм (22) является мономорфизмом, и по предложению $14(\mathrm{~b}) r_{8} \leqslant r_{8}^{Q}, r_{9} \leqslant r_{9}^{Q}$. С учетом (b) получаем, что $r_{8}=r_{8}^{Q}, r_{9}=r_{9}^{Q}$.

Предложение 17. Если в условиях предложения $16 R$ коммутативно (то есть $R$ - коммутативная область целостности), $u \in \mathscr{L}_{R} M^{\langle k\rangle}$, то $\kappa$ утверждениям предложения 16 добавляются следующие:

(a) $r_{4}=r_{5}=r_{6}=m$;

(b) $r_{7} \geqslant r_{7}^{Q}, r_{9}^{Q} \leqslant m$;

(c) $r_{18}=r_{18}^{Q}=m$;

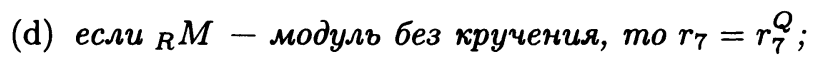

(e) если $M=R$, mo $r_{i}=r_{i}^{Q}=m, i=7,8,9, r_{i} \geqslant m, i=11,12,13,14,15$.

Доказательство. Утверждение (а) следует из предложений 14(d) и 16.

Утверждение (b) вытекает из предложений 14(d) и 13(a).

Докажем (c). Если $R$ - конечная область целостности, то $R$ - конечное поле, и равенства $r_{18}=r_{18}^{Q}=m$ следуют из предложений $13(\mathrm{~d})$ и $15(\mathrm{e})$.

Докажем (е). Пусть $\varphi: Q[\boldsymbol{x}][1 \otimes u] \rightarrow(Q \otimes M)^{t}=Q \otimes M^{t}-$ мономорфизм $Q$ модулей. Для доказательства неравенства $r_{7} \leqslant r_{7}^{Q}$ достаточно построить мономорфизм $R$-модулей $\varphi_{1}: R[\boldsymbol{x}] u \rightarrow M^{t}$. Пусть $\Pi$ - начальный параллелепипед $k$-ЛРП $u$. Тогда $R$-модуль $R[\boldsymbol{x}] u$ порождается последовательностями $\boldsymbol{x}^{i} u, i \in \Pi$, а $Q$-модуль $Q[\boldsymbol{x}][1 \otimes u]-$ последовательностями $\left[1 \otimes \boldsymbol{x}^{\boldsymbol{i}} u\right]=\boldsymbol{x}^{\boldsymbol{i}}[1 \otimes u], \boldsymbol{i} \in \Pi$. Образ $\varphi\left(\boldsymbol{x}^{\boldsymbol{i}}[1 \otimes u]\right)$, как элемент модуля $Q \otimes M^{t}$, представляется в виде

$$
\varphi\left(x^{i}[1 \otimes u]\right)=\varphi\left(\left[1 \otimes x^{i} u\right]\right)=\sum_{j} q_{i j} \otimes m_{i j}, \quad i \in \Pi,
$$

где $q_{i j} \in Q, m_{i j} \in M^{t}$. Приведем все дроби $q_{i j}$ к общему знаменателю: $q_{i j}=a_{i j} / b$, $a_{i j} \in R, b \in R \backslash 0$. Тогда

$$
\varphi\left(\left[1 \otimes x^{i} u\right]\right)=\frac{1}{b} \sum_{j} a_{i j} \otimes m_{i j}=\frac{1}{b} 1 \otimes \sum_{j} a_{i j} m_{i j}, \quad i \in \Pi .
$$


Так как $M^{t}-$ модуль без кручения, канонический гомоморфизм

$$
\beta: M^{t} \rightarrow Q \otimes M^{t}, \quad m \rightarrow 1 \otimes m,
$$

является мономорфизмом. При этом элементы $b \varphi\left(\left[1 \otimes \boldsymbol{x}^{i} u\right]\right), \boldsymbol{i} \in \Pi$, принадлежат образу $\beta\left(M^{t}\right)$. Так как $R$ коммутативно, то $b \varphi([1 \otimes v]) \in \beta\left(M^{t}\right)$ для всех $v \in R[\boldsymbol{x}] u$. Поэтому можно определить

$$
\varphi_{1}(v)=\beta^{-1}(b \varphi([1 \otimes v])), \quad v \in R[\boldsymbol{x}] u .
$$

Очевидно, $\varphi_{1}: R[\boldsymbol{x}] u \rightarrow M^{t}-$ корректно определенный гомоморфизм $R$-модулей. Проверим, что $\varphi_{1}-$ мономорфизм. Если $\varphi_{1}(v)=0$, то $b \varphi([1 \otimes v])=0$ в модуле $Q \otimes M^{t}$. Умножив на дробь $1 / b$, получим, что $\varphi([1 \otimes v])=0$. Так как $\varphi-$ мономорфизм, то $[1 \otimes v]=0$. Это означает, что $1 \otimes v(i)=0, i \in \mathbf{N}_{0}^{k}$, в модуле $Q \otimes M$. Так как отображение $\alpha$, определенное в (22), является мономорфизмом, то $v(i)=0, i \in \mathbf{N}_{0}^{k}$, то есть $v=0$. Таким образом, $\varphi_{1}-$ мономорфизм, и $r_{7} \leqslant r_{7}^{Q}$.

Докажем (е). Если $M=R$, то $Q \otimes M=Q$ и $\nu=\nu^{Q}=1$. Из предложения 16(b,c) и неравенства $r_{9}^{Q} \leqslant m$ теперь следует, что $r_{i}=r_{i}^{Q}=m, i=7,8,9$. Кроме того, по предложению 6(c) $r_{11} \geqslant r_{9}$, а по предложению $4(\mathrm{a}) r_{12} \geqslant r_{9}$. С учетом (7) получаем, что $r_{i} \geqslant m, i=11, \ldots, 15$.

Пример 11 показывает, что если ${ }_{R} M$ - модуль с кручением над коммутативной областью целостности $R$ и $k=1$, то могут выполняться неравенства $r_{i}>m$, $i=1,2,3,7, \ldots, 17$. Более того, согласно примеру 12 , неравенства $r_{i}>m, i=$ $1,2,3,10, \ldots, 15$, могут выполняться, даже если $M=R-$ коммутативная область целостности и $k=1$.

В силу предложений 16 и 17 если ${ }_{R} M$ - модуль над левой областью Ope $R$ с левым телом частных $Q$ и либо ${ }_{R} M-$ модуль без кручения, либо кольцо $R$ коммутативно, то следующие параметры $k$-ЛРП $u \in \mathscr{L}_{R} M^{\langle k\rangle}$ имеют общее значение $m:$

$$
m=\operatorname{dim}_{Q} Q[\boldsymbol{x}][1 \otimes u]=r_{4}=r_{5}=r_{6}=r_{i}^{Q}, \quad i=1, \ldots, 6,10,16 .
$$

Это значение можно полагать равным линейной сложности $k$-ЛРП над указанными модулями, если необходимо выделить какое-либо одно значение линейной сложности. В случае, когда $R$ - коммутативная область целостности и $M=R$, к этим значениям добавляется еще несколько:

$$
r_{i}=r_{i}^{Q}=m, \quad i=7,8,9 .
$$

Напомним, что если $R$ коммутативно и $k=1$, то в (15) был выделен еще один набор совпадающих значений линейной сложности:

$$
m^{\prime}=\operatorname{rank} u=r_{i}, \quad i=1,2,3,10,13,15 .
$$

Если же $R$ коммутативно, $k=1$ и $M=R$, то, согласно (16),

$$
m^{\prime}=\operatorname{rank} u=r_{i}, \quad i=1,2,3,10, \ldots, 15 .
$$

В силу предложения 16 (а) $m \leqslant m^{\prime}$. Пример 12 показывает, что если $R-$ коммутативная область целостности, $k=1$ и $M=R$, то возможна ситуация, когда $m<m^{\prime}$. В 
этом случае оба параметра $m$ и $m^{\prime}$ могут равноправно именоваться линейной сложностью 1-ЛРП $u$. Мы хотим теперь показать, что для 1-ЛРП над коммутативными областями Безу $m=m^{\prime}$.

Напомним (см. [5], стр.69), что кольцо $R$ называется левой областью Безу, если $R$ - область целостности, в которой каждый левый идеал, порожденный двумя элементами, является главным, то есть порождается одним элементом. В этом случае любой конечно порожденный левый идеал является главным. Левые области Безу являются левыми областями Оре (см. предложение 1.3 в [5]). Так как левые области Оре обладают гомоморфизмом в тело (вложение в левое тело частных), они обладают свойством ИБЧ (см. [5], стр. 22).

Кольцо $R$ называется слабо конечным, если для любого $n \in \mathbf{N}$ из $R^{n} \cong R^{n} \oplus K$ следует, что $K=0$. Слабо конечные кольца обладают свойством ИБЧ (см. [5]). Левая область Безу является слабо конечным кольцом (см. предложение 1.3 и следствие 2 теоремы 1.1 в [5]). Если кольцо $R$ обладает свойством ИБЧ, то рангом свободного модуля $R^{n}$ называется число $n$.

Лемма 4. (а) Если колъцо $R$ слабо конечно, то $r_{1}\left(R^{n}\right)=n$.

(b) Если $R$ - левая область Ope, то $r_{4}\left(R^{n}\right)=n$.

(c) Если $R$ - левая область Безу, то $r_{1}\left(R^{n}\right)=r_{4}\left(R^{n}\right)=n$.

(d) Если $R$ - левая область Безу, то любой конечно порожденный подмодуль свободного модуля свободен.

(е) Если $R$ - левал область Безу, то любой конечно порожденный подмодуль модуля $R^{n}$ является свободным модулем ранга не выше $n$.

Доказателъство. Докажем (а). Очевидно, $r_{1}\left(R^{n}\right) \leqslant n$. Предположим, что $r_{1}\left(R^{n}\right)=$ $s<n$. Тогда $R^{n}$ порождается $s$ элементами и существует эпиморфизм $\varphi: R^{s} \rightarrow R^{n}$. Так как модуль $R^{n}$ свободный и, следовательно, проективный, этот эпиморфизм расщепляется (см. теорему 5.3.1(b) в [4]), то есть

$$
R^{s}=\operatorname{Ker} \varphi+L .
$$

Ограничение $\varphi$ на $L$ есть изоморфизм между $L$ и $R^{n}$. Следовательно,

$$
R^{s} \cong \operatorname{Ker} \varphi \oplus R^{n} \cong\left(\operatorname{Ker} \varphi \oplus R^{n-s}\right) \oplus R^{s}
$$

Так как кольцо $R$ слабо конечно, то $\operatorname{Ker} \varphi \oplus R^{n-s}=0$, что невозможно, поскольку $s<n$. Следовательно, $r_{1}\left(R^{n}\right)=n$.

Доказательство (b) см. в [5], стр. 48.

Утверждение (c) следует из (a) и (b).

Для доказательства (d) заметим, что в силу предложения 1.3 в [5] $R$ является полу-FI-кольцом, и тогда согласно теорему $1.1(\mathrm{e})$ в [5] любой конечно порожденный подмодуль свободного модуля свободен.

Утверждение (f) содержится в предложении 1.4 на стр. 69 в [5].

Предложение 18. Пусть $R$ - левая область Безу, $M-$ свободный модулъ ранга

$$
\nu \in \mathbf{N} \cup\{\infty\}, \quad u \in \mathscr{L}_{R} M^{\langle k\rangle},
$$


$m$ - параметр, введеннъй в предложении 16. Тогда модуль сдвигов $R[\boldsymbol{x}]$ и является свободным $R$-модулем ранга $m$ и к соотношениям предложений 16, 17 добавляются следугщие:

$$
\left.r_{1}=r_{16}=m, \quad r_{7}=\right] m / \nu[
$$

где при $\nu=\infty$ вместо $] m / \nu[$ нужно брать 0 , если $m=0, u 1$, если $m \geqslant 1$.

Доказателъство. В силу предложений $16(\mathrm{~b})$ и $\left.17(\mathrm{~d}) r_{7}=r_{7}^{Q}=\right] m / \nu^{Q}[$. По лемме $4(\mathrm{~b}) r_{1}\left({ }_{R} M\right)=r_{4}\left({ }_{R} M\right)$, и ввиду предложения $16 \nu^{Q}=\nu$. Следовательно, $\left.r_{7}=\right] m / \nu[$.

Пусть $R[\boldsymbol{x}] u \rightarrow M^{t}-$ мономорфизм (где, например, $t=r_{7}$ ). По лемме 4(d) $R[\boldsymbol{x}] u$ - свободный модуль. Пусть $n \in \mathbf{N}_{0} \cup\{\infty\}$ - его ранг. По лемме 4(c)

$$
n=r_{1}(R[\boldsymbol{x}] u)=r_{4}(R[\boldsymbol{x}] u),
$$

то есть $n=r_{1}(u)=r_{4}(u)$. Так как $r_{4}(u)=m$ по предложению $16(\mathrm{c})$, то $R[\boldsymbol{x}] u-$ свободный модуль ранга $m$ и $r_{1}=m$.

Ввиду (8) $r_{16} \leqslant r_{1}=m$, а по предложению 16(а) $r_{16} \geqslant m$. Следовательно, $r_{16}=m$.

Пример 12 показывает, что в случае, когда $M=R-$ коммутативная область целостности, $k=1, \nu=1$, соотношения $r_{1}=m$ и $\left.r_{7}=\right] m / \nu[=m$ могут быть неверными, то есть условие предложения 18 является существенным. Как следствие, отсюда получаем, что коммутативная область целостности $R$ из примера 12 не является областью Безу.

Предложение 19. Если в условиях предложения 18 кольцо $R$ коммутативно, то $r_{17} \geqslant m$.

Доказательство. Пусть $u \in L_{M}(I)$, где $I$ - унитарный идеал кольца $R[\boldsymbol{x}]$, $r_{1}\left(L_{M}(I)\right)=r_{17}$. Если $G_{1}\left(x_{1}\right), \ldots, G_{k}\left(x_{k}\right)$ - элементарные характеристические многочлены идеала $I$, то, ввиду коммутативности $R$,

$$
R[\boldsymbol{x}] u \subseteq L_{M}(I) \subseteq L_{M}(\mathbf{G})
$$

По лемме 4(d) $L_{M}(I)$ - свободный модуль. По лемме 4(c) его ранг равен $r_{1}\left(L_{M}(I)\right)=$ $r_{17}$. Теперь по лемме $4(\mathrm{e})$ из включения $R[\boldsymbol{x}] u \subseteq L_{M}(I)$ следует, что $R[\boldsymbol{x}] u$ - свободный модуль ранга не выше $r_{17}$, то есть $m \leqslant r_{17}$.

Рассмотрим теперь 1-ЛРП над коммутативными областями Безу.

Предложение 20. Пусть $M-$ свободный модуль ранга $\nu \in \mathbf{N} \cup\{\infty\}$ над коммутативной областъю Безу $R$ с полем частных $Q, k=1, u \in \mathscr{L}_{R} M^{\langle 1\rangle}, m=$ $\operatorname{dim}_{Q} Q[\boldsymbol{x}][1 \otimes u]-$ параметр, введенный в предложении 16. Тогда

$$
\begin{aligned}
& r_{i}=r_{i}^{Q}=m, \quad i=1, \ldots, 6,10,13,15,16,18, \\
r_{7}= & \left.r_{7}^{Q}=\right] m / \nu\left[, \quad r_{11} \leqslant m, \quad r_{12} \leqslant r_{14} \leqslant m, \quad r_{17} \geqslant m,\right.
\end{aligned}
$$

где при $\nu=\infty$ вместо $] m / \nu[$ нужно брать 0 , если $m=0, u 1$, если $m \geqslant 1$.

Минимальный многочлен 1-ЛРП и над кольцом $R$ определен однозначно и совпадает с минималъным многочленом 1-ЛРП $[1 \otimes u]$ над полем $Q$.

Eсли $\propto$ тому же $M=R$, то

$$
r_{i}=r_{i}^{Q}=m, \quad i=1, \ldots, 18
$$


Доказательство. В силу предложений 12 и $13(\mathrm{~d}, \mathrm{e})$

$$
r_{i}^{Q}=m, \quad i=1, \ldots, 6,10,11,13,15,16,18 .
$$

В предложениях 16(c), 17(c,d), 18 и 19 доказано, что

$$
\left.r_{1}=r_{4}=r_{5}=r_{6}=r_{16}=r_{18}=m, \quad r_{7}=r_{7}^{Q}=\right] m / \nu\left[, \quad r_{17} \geqslant m .\right.
$$

По предложению $11(\mathrm{a})$

$$
r_{1}=r_{2}=r_{3}=r_{10}=r_{13}=r_{15}
$$

Следовательно, $r_{i}=m, i=1, \ldots, 6,10,13,15,16,18$. По предложению $9 r_{11} \leqslant r_{10}=$ $m$, и в силу (7) $r_{12} \leqslant r_{14} \leqslant r_{15}=m$.

По предложению 10 минимальный многочлен ЛРП $u$ над кольцом $R$ определен однозначно. В силу предложения 14 он является характеристическим многочленом ЛРП $[1 \otimes u]$. Так как $r_{10}=r_{10}^{Q}$, он является минимальным многочленом ЛРП $[1 \otimes u]$ над полем $Q$.

Если $M=R$, то по предложению $13(\mathrm{f}) r_{i}^{Q}=m, i=1, \ldots, 18$. Из уже доказанного и предложения $17(\mathrm{e})$ вытекает, что $r_{i}=m, i=1, \ldots, 16,18$. Пусть $F(x)$ - минимальный многочлен ЛРП $u$ над кольцом $R$. Тогда $\operatorname{deg} F(x)=r_{10}=m$ и $u \in L_{R}(F) \cong R^{m}$. Следовательно, $r_{17}(u) \leqslant r_{1}\left(L_{R}(F)\right) \leqslant m$. В итоге $r_{17}=m$.

Предложение 20 показывает, что коммутативные области Безу являются классом колец, для 1-ЛІРП над которыми свойства линейной сложности оказываются наиболее близкими к свойствам линейной сложности 1-ЛРП над полем. Этот класс колец включает (как собственное подмножество) коммутативные области главных идеалов, в частности, кольцо целых чисел Z. Ключевое свойство областей Безу, использовавшееся в доказательствах, заключается в том, что конечно порожденный подмодуль свободного модуля над левой областью Безу является свободным. Для коммутативных областей Безу это свойство является характеристическим (см. [5], стр. 69).

\section{2. Достаточные условия равенства $r_{4}=r_{5}=r_{6}$}

Если $R$ - левая область Оре, в частности, коммутативная область целостности, то по предложению 16(c) для $k$-ЛРП над $R$ выполняются равенства $r_{4}=r_{5}=r_{6}$. Здесь мы укажем еще один класс коммутативных колец (пересечение которого с классом областей целостности совпадает с полями), для ЛРП над которыми эти равенства также верны.

Предложение 21. Пусть $R$ - коммутативное локалъное колъцо с максимальным идеалом $J$ такое, что $\operatorname{An}(J) \neq 0, u \in \mathscr{L}_{R} R^{\langle k\rangle}$. Тогда $r_{4}=r_{5}=r_{6}$.

Доказательство. Пусть последовательности $v_{1}, \ldots, v_{n} \in R[\boldsymbol{x}] u$ линейно независимы, $n=r_{4}$. Если $F_{1}\left(x_{1}\right), \ldots, F_{k}\left(x_{k}\right)$ - элементарные характеристические многочлены $k$-ЛРП $u$ и $\Pi$ - ее начальный параллелепипед, $|\Pi|=m$, то по предложению $2(\mathrm{~b})$ векторы $v_{1}[\Pi], \ldots, v_{n}[\Pi] \in R^{\Pi}=R^{m}$ линейно независимы над $R$. Составим из этих векторов (столбцов) $m \times n$ матрицу $V=\left(v_{1}[\Pi], \ldots, v_{n}[\Pi]\right)$. Так как столбцы этой матрицы линейно независимы, система линейных уравнений $V \boldsymbol{x}=\mathbf{0}$ имеет только 
нулевое решение. В силу [18] (см. стр.69) (см. также теорему 5 в [3]) $\mathrm{rk} V=n$, то есть $\operatorname{An}\left(I_{V}(n)\right)=0$, где $I_{V}(n)$ - идеал кольца $R$, порожденный всеми минорами матрицы $V$ порядка $n$. Если $I_{V}(n) \neq R$, то $I_{V}(n) \subseteq J$, следовательно, $\operatorname{An}\left(I_{V}(n)\right) \supseteq \operatorname{An}(J) \neq 0$, получаем противоречие. Поэтому $I_{V}(n)=R$. Поскольку $R$ локально, это означает, что существует минор матрицы $V$ порядка $n$, являющийся обратимым элементом кольца $R$.

Пусть $\bar{a}, \bar{F}(x), \bar{u}, \bar{V}$ - образы элемента, многочлена, последовательности и матрицы над $R$ в поле вычетов $\bar{R}=R / J$ кольца $R$. Матрица $\bar{V}$ содержит ненулевой минор порядка $n$. Следовательно, ее столбцы $\bar{v}_{1}[\Pi], \ldots, \bar{v}_{n}[\Pi]$ линейно независимы над полем $\bar{R}$. Поэтому последовательности $\bar{v}_{1}, \ldots, \bar{v}_{n} \in \bar{R}[\boldsymbol{x}] \bar{u}$ линейно независимы над $\bar{R}$, и $r_{4}(\bar{u}) \geqslant n$, где $r_{4}(\bar{u})$ - линейная сложность $k$-ЛРП $\bar{u}$ над полем $\bar{R}$. По предложению $12(\mathrm{a}) r_{4}(\bar{u})=r_{6}(\bar{u})$. Следовательно, $r_{6}(\bar{u}) \geqslant n$. Это означает, что существует диаграмма Ферре $\mathscr{F}$ мощности $|\mathscr{F}|=r_{6}(\bar{u}) \geqslant n$ такая, что последовательности $\left(\boldsymbol{x}^{i} \bar{u}, \boldsymbol{i} \in \mathscr{F}\right)$ линейно независимы над $\bar{R}$. Очевидно, что $\bar{F}_{1}\left(x_{1}\right), \ldots, \bar{F}_{k}\left(x_{k}\right)$ - элементарные характеристические многочлены $k$-ЛРП $\bar{u}$ и $\Pi$ - ее начальный параллелепипед. По предложению 2(b) векторы $\left(x^{i} \bar{u}[\Pi], i \in \mathscr{F}\right)$ из $\bar{R}^{\Pi}$ линейно независимы над $\bar{R}$. Рассмотрим векторы (столбцы) $\left(x^{i} u[\Pi], i \in \mathscr{F}\right)$ из $R^{\Pi}=R^{m}$ и составим из них $m \times|\mathscr{F}|$ матрицу $U=\left(\boldsymbol{x}^{i} u[\Pi], i \in \mathscr{F}\right)$. Так как столбцы матрицы $\bar{U}$ линейно независимы над $\bar{R}$, матрица $\bar{U}$ содержит ненулевой минор порядка $|\mathscr{F}|$. Следовательно, $U$ содержит минор порядка $|\mathscr{F}|$, являющийся обратимым элементом кольца $R$. Отсюда, $\operatorname{rk} U=|\mathscr{F}|$, и в силу [18], (см. стр. 159) (или теоремы 5 в [3]) система линейных уравнений $U \boldsymbol{x}=\mathbf{0}$ имеет только нулевое решение. Это означает, что столбцы матрицы $U$ линейно независимы над $R$. Поэтому последовательности $\left(\boldsymbol{x}^{i} u, i \in \mathscr{F}\right)$ линейно независимы над $R$. Следовательно, $r_{6} \geqslant|\mathscr{F}|=r_{6}(\bar{u}) \geqslant n=r_{4}$. Так как $r_{4} \geqslant r_{5} \geqslant r_{6}$ ввиду (1), то $r_{4}=r_{5}=r_{6}$.

Следствие 6. Если $R$ - коммутативное локальное артиново (в частности, конечное) колъцо, $u \in \mathscr{L}_{R} R^{\langle k\rangle}$, mо $r_{4}=r_{5}=r_{6}$.

Доказательство. По теореме 9.3.9 в [4] радикал $J$ артинова кольца нильпотентен, поэтому $\operatorname{An}(J) \neq 0$.

Всякое коммутативное артиново кольцо является прямой суммой локальных (см. теорему 8.7 в [1]), поэтому следствие 6 естественно попытаться обобщить на коммутативные артиновы кольца. Однако, если

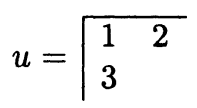

- 2-последовательность над кольцом $Z_{6}$, рассмотренная в примере 4 , то $r_{4}=2$, $r_{5}=r_{6}=1$, то есть соотношения $r_{4}=r_{5}=r_{6}$ не выполняются. Тем не менее, для 1-последовательностей указанное обобщение следствия 6 верно.

Предложение 22. Если $R=R^{(1)} \oplus R^{(2)}$ и для 1-последователъностей над колъцами $R^{(1)}$ и $R^{(2)}$ выполняются равенства $r_{4}=r_{5}=r_{6}$, то для 1-последователъностей над кольцом $R$ также выполняются равенства $r_{4}=r_{5}=r_{6}$.

Доказательство. Ввиду (1) $r_{4} \geqslant r_{5} \geqslant r_{6}$. Докажем, что $r_{6}(u) \geqslant r_{4}(u)$. Каждый элемент $c \in R$ и последовательность $u \in R^{\langle 1\rangle}$ однозначно представляется в виде

$$
c=c^{(1)}+c^{(2)}, \quad u=u^{(1)}+u^{(2)},
$$


где $c^{(i)}, u^{(i)}$ - элемент и последовательность над кольцом $R^{(i)}, i=1,2$. Пусть $u-$ произвольная ЛРП над $R$, и пусть последовательности $v_{1}, \ldots, v_{n} \in R[\boldsymbol{x}] u$ линейно независимы над $R, n=r_{4}(u)$. Тогда последовательности $v_{1}^{(i)}, \ldots, v_{n}^{(i)} \in R^{(i)}[\boldsymbol{x}] u^{(i)}$. линейно независимы над $R^{(i)}, i=1,2$, поскольку если

$$
\sum_{s} a_{s} v_{s}^{(i)}=0
$$

для некоторого $i=1,2$, где $a_{s} \in R^{(i)}$, то

$$
\sum_{s=1}^{n} a_{s} v_{s}=\sum_{s=1}^{n} a_{s}\left(v_{s}^{(1)}+v_{s}^{(2)}\right)=\sum_{s=1}^{n} a_{s} v_{s}^{(i)}=0 .
$$

Так как по условию $r_{4}\left(u^{(i)}\right)=r_{6}\left(u^{(i)}\right)$, последовательности

$$
u^{(i)}, x u^{(i)}, \ldots, x^{n-1} u^{(i)}
$$

линейно независимы над $R^{(i)}, i=1,2$. Но тогда последовательности

$$
u, x u, \ldots, x^{n-1} u
$$

линейно независимы над $R$ (два последних утверждения используют условие $k=1$ ). Действительно, если

$$
\sum_{t=0}^{n-1} b_{t} x^{t} u=0, \quad b_{t} \in R
$$

TO

$$
\sum_{t=0}^{n-1} b_{t}^{(i)} x^{t} u^{(i)}=0, \quad i=1,2
$$

откуда, $b_{t}^{(i)}=0, t=0,1, \ldots, n-1, i=1,2$, и следовательно, $b_{t}=0, t=0,1, \ldots, n-1$. Этим доказано, что $r_{6}(u) \geqslant n$, то есть $r_{6}(u) \geqslant r_{4}(u)$.

Следствие 7. Рассмотрим следующие классы коммутативных колец:

(а) локальные колъца, у которых аннулятор максимального идеала не равен 0 ;

(b) артиновы (в частности, конечные) кольца;

(c) области целостности.

Если кольцо $R$ есть прямая сумма колец из этих классов, то для любой 1последовательности $u \in \mathscr{L}_{R} R^{\langle 1\rangle}$ выполняются соотношения

$$
\begin{aligned}
r_{4} & =r_{5}=r_{6} \leqslant r_{18} \leqslant r_{7} \leqslant r_{8} \leqslant r_{9} \leqslant r_{1} \\
& =r_{2}=r_{3}=r_{10}=r_{11}=r_{12}=r_{13}=r_{14}=r_{15}=\operatorname{rank} u
\end{aligned}
$$

Доказательство. Равенства $r_{4}=r_{5}=r_{6}$ в случаях (a), (b) следуют из предложений 21 и 22, в случае (c) - из предложения 16(c). Остальные соотношения вытекают из следствия 3. 
Пример 3 показывает, что для 1-ЛРП над конечным локальным кольцом $Z_{4}$ неравенства $r_{6} \leqslant r_{18} \leqslant r_{7}$ могут быть строгими. Согласно примеру 12 для 1-ЛРП над коммутативной областью целостности неравенство $r_{9} \leqslant r_{1}$ может быть строгим.

Подводя итог, отметим, что для последовательностей над коммутативными кольцами имеются две серии совпадений различных определений линейной сложности. Первая из них:

$$
m=r_{1}=r_{2}=r_{3}=r_{10}=r_{13}=r_{15}=\operatorname{rank} u
$$

справедлива для 1-ЛРП $u \in \mathscr{L}_{R} M^{\langle 1\rangle}$, где $R-$ произвольное коммутативное кольцо. Если при этом $M=R$, то к указанным равенствам добавляются равенства $m=r_{11}=r_{12}=r_{14}$ (см. следствие 3). Вторая серия совпадений $r_{4}=r_{5}=r_{6}$ справедлива для $k$-ЛРП $u \in \mathscr{L}_{R} M^{\langle k\rangle}$, где $R$ - коммутативная область целостности и $M$ - произвольный $R$-модуль (предложение $17(\mathrm{a}))$ или $R$ - левая область Оре и $M$ - модуль без кручения (предложение $16(\mathrm{c})$ ), а также для 1 -ЛРП $u \in \mathscr{L}_{R} R^{\langle 1\rangle}$, где $R$ - коммутативное артиново кольцо (следствие 7). Для 1-ЛРП над коммутативными артиновыми кольцами или над коммутативными областями целостности эти две серии совпадений могут быть различными, то есть $r_{4}<r_{1}$ (примеры 3,12 ). Для 1-ЛРП над коммутативными областями Безу (в частности, коммутативными областями главных идеалов) и свободными модулями над ними эти две серии равны друг другу. Заметим сходство этой ситуации с той, которая возникает при определении ранга модуля над коммутативным кольцом. В качестве ранга модуля $M$ обычно выбирают один из параметров $r_{1}(M)$ или $r_{4}(M)$, введенных в разделе 3 , причем $r_{4}(M)$ используется для модулей над областями целостности (см. стр. 49 в [13]). В общем случае $r_{1}(M) \neq r_{4}(M)$, но если $M-$ свободный модуль над коммутативной областью Безу, то $r_{1}(M)=r_{4}(M)$ (лемма $\left.4(\mathrm{c})\right)$.

\section{3. Заключение}

Полученные результаты и примеры показывают, что большинство из введенных определений линейной сложности в общем случае не эквивалентны друг другу. Трудно (и, видимо, не нужно) выделить какой-либо один параметр и именно его назвать линейной сложностью $k$-ЛРП. Все введенные определения являются естественными, и каждое из них может оказаться полезным при решении конкретных задач.

При рассмотрении $k$-ЛРП над различными классами колец и модулей некоторые из определений линейной сложности оказываются эквивалентными. С этой точки зрения, помимо полей и тел, в работе выделено несколько таких классов колец: левые области Оре, коммутативные артиновы кольца и коммутативные области Безу. Так, если $u \in \mathscr{L} R^{\langle 1\rangle}-1$-ЛРП над коммутативным артиновым (в частности, конечным) кольцом $R$, то справедливы следующие соотношения (см. следствия 3 и 7 ):

$$
\begin{gathered}
r_{4}=r_{5}=r_{6} \leqslant r_{18} \leqslant r_{7} \leqslant r_{8} \leqslant r_{9} \leqslant \operatorname{rank} u=r_{i}, \quad i=1,2,3,10, \ldots, 15, \\
r_{16} \leqslant r_{1}, \quad r_{17} \leqslant r_{1} .
\end{gathered}
$$

Если же $u$ - 1-ЛРП над коммутативной областью Безу $R$, то так же, как для ЛРП над полем, $u$ имеет единственный минимальный многочлен и все 18 рассматриваемых определений линейной сложности эквивалентны (предложение 20):

$$
r_{1}(u)=r_{2}(u)=\ldots=r_{18}(u)
$$


Таким образом, коммутативные области Безу являются классом колец, для которых теория линейной сложности 1-последовательностей наиболее полно повторяет классическую теорию линейной сложности 1-последовательностей над полем.

Работа оставляет множество нерешенных задач. Это вызвано в том числе и количественными соображениями: требовалось попарно сравнить большое число различных определений. Перечислим некоторые задачи.

(1) Для любой пары $r_{i}, r_{j}$ охарактеризовать класс колец и модулей, над которыми выполняется одно из утверждений $r_{i} \leqslant r_{j}, r_{i}=r_{j}, r_{i} \geqslant r_{j}$.

(2) Обобщить результаты о линейной сложности последовательностей над телами на левые области Безу (подобно коммутативному случаю).

(3) Рассмотреть более подробно случай конечных колец и модулей, а также случай артиновых и нетеровых колец.

(4) Исследовать поведение линейной сложности при переходе к фактормодулям, прямым суммам и произведениям, локализациям и модулям частных (для колец, не являющихся областями Оре).

(5) Выяснить, являются ли коммутативные области Безу наибольшим классом колец, для 1-ЛРП над которыми все 18 определений линейной сложности совпадают.

(6) Описать кольца и модули, над которыми $r_{6}=r_{10}$, то есть каждая 1-ЛРП имеет единственный минимальный многочлен (см. предложение 10 и следствие 4).

(7) Доказать или опровергнуть равенства $r_{2}=r_{3}, r_{5}=r_{6}, r_{8}=r_{9}$.

(8) Исследовать параметры $r_{19}, r_{20}, r_{21}$.

(9) Для каких $i \in\{1, \ldots, 21\}$ верно утверждение: последовательность $u \in M^{\langle k\rangle}$ есть $k$-ЛРП тогда и только тогда, когда $r_{i}(u)<\infty$ ?

(10) Выяснить, является ли 2-ЛРП из примера 9 выходом 2-линейного регистра сдвига, то есть 2-ЛРС последовательностью.

В заключение приведем перечень всех полученных результатов в виде двух таблиц: таблицы результатов и таблицы примеров. В первом столбце каждой таблицы находится номер формулы, предложения или примера, из которого взяты приводимые соотношения. Во втором столбце приведены краткие условия на кольцо $R$, модуль $M$, параметр $k$ и последовательность $u$ (полная информация находится в тексте работы). Остальные столбцы занумерованы числами $1, \ldots, 18$, означающими линейную сложность $r_{1}, \ldots, r_{18}$. В случае, когда $R-$ левая область Оре, столбец $r_{1}^{Q}$ в таблице примеров содержит линейную сложность $r_{1}^{Q}(1 \otimes u)$ последовательности $[1 \otimes u]$ над модулем частных $Q \otimes M$ (или линейную сложность $r_{1}^{Q}(u)$ последовательности $u$ над телом частных $Q$, если $u$ - последовательность над кольцом $R$ ). 
Таблица 1. Таблица результатов

\begin{tabular}{|c|c|c|c|c|c|c|c|c|c|c|c|c|c|c|c|c|c|c|c|}
\hline Noo & Условия & 1 & \begin{tabular}{|l|}
2 \\
$\Omega$ \\
\end{tabular} & \begin{tabular}{|l|}
3 \\
$\mathscr{F}$
\end{tabular} & 4 & $\begin{array}{l}5 \\
\Omega\end{array}$ & $\begin{array}{l}6 \\
\mathscr{F}\end{array}$ & 7 & \begin{tabular}{|l|}
8 \\
$\Omega$ \\
\end{tabular} & \begin{tabular}{|l|}
9 \\
$\mathscr{F}$
\end{tabular} & $\begin{array}{l}10 \\
\Phi\end{array}$ & $\left|\begin{array}{c}11 \\
\langle\chi, \mathscr{F}\rangle\end{array}\right|$ & 12 & 13 & 14 & 15 & 16 & $\begin{array}{c}17 \\
L(I)\end{array}$ & \begin{tabular}{|c|}
18 \\
$\log$ \\
\end{tabular} \\
\hline $\begin{array}{l}(8) \\
1 \\
2 \\
3 \\
4 \\
5\end{array}$ & $\mid \begin{array}{l}R \text { комм. } \\
M \text { конечен, }|M| \leqslant|R|\end{array}$ & $\begin{array}{l}\geqslant 16 \\
=1^{\prime} \\
=1^{\Pi} \\
\geqslant 18\end{array}$ & $=2^{\prime}$ & $\begin{array}{l}=3^{\prime} \\
=10\end{array}$ & $\begin{array}{l}=4^{\prime} \\
=4^{\Pi} \\
\leqslant 18 \\
\leqslant 7\end{array}$ & $\geqslant 5$ & $=6^{\prime}$ & $\geqslant 18 / l$ & $\leqslant 8 \leqslant$ & $\leqslant 12$ & $\begin{array}{l}=3 \\
\leqslant 13\end{array}$ & $\leqslant 15$ & $\leqslant 14$ & $\leqslant 15$ & $\begin{array}{l}14 \\
\geqslant 12\end{array}$ & $\begin{array}{l}\leqslant 15 \\
\geqslant 13\end{array}$ & $\leqslant 1$ & & $\begin{array}{l}\geqslant 4 \\
\leqslant 1 \\
\leqslant 7 l\end{array}$ \\
\hline 8 & $\begin{array}{l}R \text { комм. } \\
R \text { комм. или артин. слева } \\
R \text { комм., } M-\text { Е-модуль }\end{array}$ & $2 \geqslant 4$ & $\geqslant 8$ & $\geqslant 9$ & $\mid \begin{array}{l}\leqslant 1 \\
\leqslant 7 \nu\end{array}$ & & & $\geqslant 4 / \nu$ & $\leqslant 2$ & $\begin{array}{l}\leqslant 3 \\
\leqslant 11\end{array}$ & & $\begin{array}{c}\geqslant 14 \\
\geqslant 9 \\
\geqslant 16 / \nu\end{array}$ & $\begin{array}{c}\geqslant \sqrt[k]{13} / \nu \\
12=\end{array}$ & $\leqslant(12 \nu)^{k}$ & $\leqslant 11$ & $=15$ & $\leqslant 11 \nu$ & & \\
\hline $\begin{array}{l}9 \\
10 \\
11 \\
3\end{array}$ & $\begin{array}{l}k=1 \\
k=1 \\
k=1, R \text { комм. } \\
k=1, R \text { комм., } M=R\end{array}$ & $\begin{aligned} & r_{6} \\
= & m \\
= & m\end{aligned}$ & $\begin{aligned} & \\
= & r_{1} \\
= & m \\
= & m\end{aligned}$ & $\begin{array}{l}10 \\
=m \\
=m\end{array}$ & $\Longleftrightarrow$ & $\exists ! m$ & $\begin{array}{l}\leqslant 10 \\
2_{u}(x)\end{array}$ & $3 \leqslant 7 \leqslant 8$ & $\leqslant 9 \leqslant n$ & & $\begin{array}{l}\geqslant 6 \\
\geqslant 11 \\
=m \\
=m\end{array}$ & $=m$ & $=m$ & $\begin{array}{l}=m \\
=m\end{array}$ & $=m$ & $\begin{array}{l}=m \\
=m\end{array}$ & $\leqslant m$ & $\begin{array}{l}\leqslant m \nu \\
\leqslant m\end{array}$ & $\leqslant m$ \\
\hline $\begin{array}{l}12 \\
13\end{array}$ & $\begin{array}{l}R \text { тело } \\
R \text { тело, } k=1 \\
R \text { поле } \\
R \text { поле, } k=1 \\
R \text { поле, } M=R \\
R \text { поле, } M=R\end{array}$ & $\begin{array}{l}=m \\
=m\end{array}$ & $\begin{array}{l}=m \\
\text { об }\end{array}$ & $\begin{array}{l}=m \\
\text { IOCTI }\end{array}$ & $\begin{array}{l}=m \\
=m\end{array}$ & $\begin{array}{l}=m \\
\text { ия } k\end{array}$ & $\begin{array}{l}=m \\
=m\end{array}$ & $\begin{array}{l}=] \frac{m}{\nu}[ \\
=] \frac{m}{\nu}[ \\
=m\end{array}$ & $\begin{array}{l}\geqslant 1 \frac{m}{\nu} \\
\geqslant 1 \frac{m}{\nu} \\
\leqslant m\end{array} \mid$ & $\begin{array}{l}\geqslant] \frac{m}{\nu} \\
\geqslant] \frac{m}{\nu} \\
\leqslant m\end{array}$ & $=m$ & $\begin{array}{c}=m \\
\geqslant m / \nu\end{array}$ & $=m$ & $\begin{array}{l}\geqslant m \\
\geqslant m \\
=m \\
=m\end{array}$ & $=m$ & $\begin{array}{l}=m \\
=m\end{array}$ & $\begin{array}{l}=m \\
=m\end{array}$ & $\begin{array}{l}\geqslant m \\
\leqslant m \nu \\
=m \nu \\
=m\end{array}$ & $=m$ \\
\hline
\end{tabular}


Таблица 2. Продолжение таблицы 1

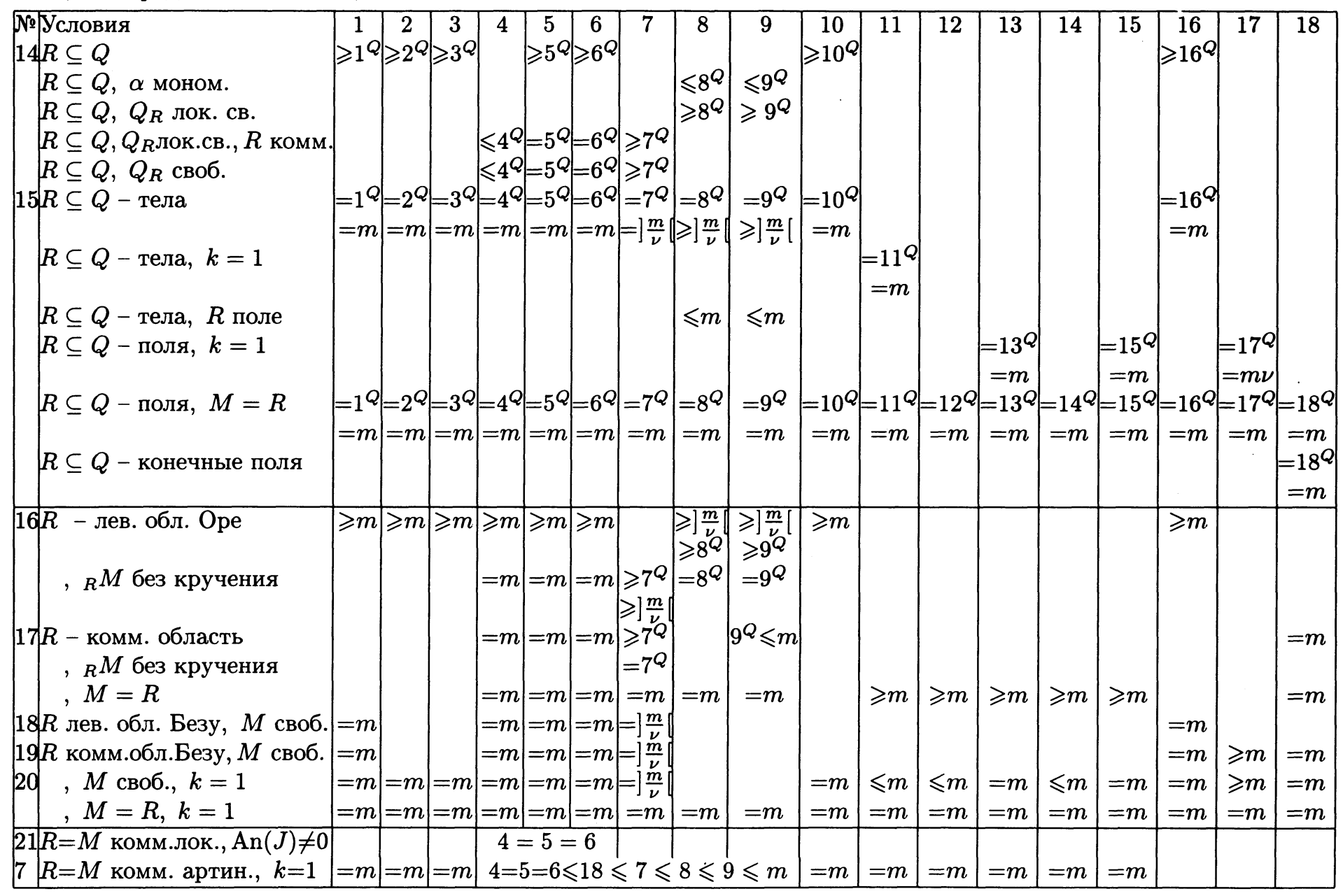


Таблица 3. Таблица примеров

\begin{tabular}{|c|c|c|c|c|c|c|c|c|c|c|c|c|c|c|c|c|c|c|c|c|c|}
\hline \multicolumn{3}{|c|}{ № Условия } & & $\begin{array}{l}2 \\
\Omega\end{array}$ & $\begin{array}{l}3 \\
\mathscr{F}\end{array}$ & 4 & $\begin{array}{l}5 \\
\Omega\end{array}$ & $\begin{array}{l}6 \\
\mathscr{F}\end{array}$ & 7 & $\begin{array}{l}8 \\
\Omega\end{array}$ & $\begin{array}{l}9 \\
\mathscr{F}\end{array}$ & $\begin{array}{c}10 \\
\Phi\end{array}$ & $\left\{\begin{array}{l}11 \\
\langle\chi,\end{array}\right.$ & $\begin{array}{l}12 \\
\mathscr{F}\rangle\end{array}$ & 13 & 14 & & \multicolumn{4}{|c|}{$L(I) \log$} \\
\hline & $R \cong R^{2}, u=$ & $(1,0,0, \ldots)$ & 1 & 1 & 1 & $\infty$ & 1 & 1 & 1 & 1 & 1 & 1 & 1 & 1 & 1 & 1 & \begin{tabular}{|l|l|}
1 & \\
\end{tabular} & 1 & 1 & - & \\
\hline & $R \cong R^{2}, u=$ & $(0,1,0, \ldots)$ & 1 & 2 & 2 & $\infty$ & 2 & 2 & 1 & 2 & 2 & 2 & 2 & 2 & 2 & 2 & 2 & 1 & 1 & - & \\
\hline & $R=Z_{4}, u=$ & $(2,2,2, \ldots)$ & 1 & 1 & 1 & 0 & 0 & 0 & 1 & 1 & 1 & 1 & 1 & 1 & 1 & 1 & 1 & 1 & 1 & $1 / 2$ & \\
\hline & $R=\mathbf{Z}, u=$ & $\begin{array}{ll}1 & 2 \\
3 & \end{array}$ & 2 & 3 & 3 & 2 & 2 & 2 & 2 & 2 & 2 & 3 & 3 & 3 & 3 & 3 & 3 & 2 & & - & 2 \\
\hline & $R=Z_{6}$ & & 2 & 3 & 3 & 2 & 1 & 1 & 2 & 3 & 3 & 3 & 3 & 3 & 3 & 3 & 3 & 2 & & 2 & \\
\hline 5 & $R$ поле, $M$ & $=R^{2}, u=\left(\begin{array}{c}\alpha^{i} \\
\beta^{i}\end{array}\right)$ & 2 & 2 & 2 & 2 & 2 & 2 & 1 & 1 & 1 & 2 & 2 & 1 & 2 & 1 & 2 & 2 & 4 & 2 & 2 \\
\hline 6 & $a b \notin b R, u=$ & $\left(a^{i} b\right)$ & 1 & 1 & 1 & & 1 & 1 & 1 & & & 1 & 1 & $>1$ & $>$ & $>1$ & $>$ & 1 & 1 & - & \\
\hline 7 & $R=P[\alpha, \beta] /($ & $\left.\alpha^{2}, \beta^{2}\right), u=(0, \alpha \beta, 0, \ldots)$ & 2 & 2 & 2 & 0 & 0 & 0 & 2 & 2 & 2 & 2 & 2 & 2 & 2 & 2 & 2 & 1 & & & 0 \\
\hline & $c_{i} a^{i} b=0, c_{i}$ & $a^{i+1} b \neq 0, u=\left(a^{i} b\right)$ & 1 & 1 & 1 & & 1 & 1 & 1 & $>1$ & $>1$ & 1 & 1 & & & & & 1 & 1 & - & \\
\hline & $R=P\langle\alpha, \beta$ & $\gamma\rangle / I, u=\left(\alpha^{i} \beta^{j} \gamma\right)$ & 1 & 1 & 1 & & 1 & 1 & & & & 1 & $>1$ & & & & & 1 & & - & \\
\hline & $R$ поле, $M=$ & $R^{2}, u=\left(\left(\begin{array}{l}0 \\
0\end{array}\right),\left(\begin{array}{l}1 \\
0\end{array}\right),\left(\begin{array}{l}0 \\
0\end{array}\right), \ldots\right)$ & 2 & 2 & 2 & 2 & 2 & 2 & 1 & 2 & 2 & 2 & 2 & 2 & 2 & 2 & 2 & 2 & 4 & 2 & 2 \\
\hline & $R=\mathbf{Z}, M=$ & $\mathbf{Z}_{4}, u=(2,2,2, \ldots)$ & 1 & 1 & 1 & 0 & 0 & 0 & 1 & 1 & 1 & 1 & 1 & 1 & 1 & 1 & 1 & 1 & 1 & - & 0 \\
\hline & $R=P[\alpha, \beta] /($ & $\left.\alpha^{2}-\beta^{3}\right), u=(\beta, \alpha, \ldots)$ & 2 & 2 & 2 & 1 & 1 & 1 & 1 & 1 & 1 & 2 & 2 & 2 & 2 & 2 & 2 & & & - & 1 \\
\hline
\end{tabular}


Для краткости в таблице результатов обозначение $r_{i}$ заменено на $i$, так что каждое число $i=1, \ldots, 18$ нужно заменять на символы $r_{i}$. Например, записи $\leqslant 2,=8^{Q}$, $\geqslant m / \nu, \geqslant 1 / \nu$ в столбце 8 таблицы результатов обозначают соответственно $r_{8} \leqslant r_{2}$,

$r_{8}=r_{8}^{Q}, r_{8} \geqslant m / \nu, r_{8} \geqslant r_{1} / \nu$ ( $m$ и $\nu$ не числа, а буквы $)$. В таблице результатов также используются следующие обозначения: $\left.\nu=r_{1}(M), l=\log _{|R|}|M|,\right] x[-$ наименьшее натуральное число, большее или равное $x$ (при этом, если $\nu=\infty$, то вместо ] $m / \nu[$ нужно брать 0 при $m=0$ и 1 при $m \geqslant 1$, см. предложения 12(b), 13(a)), $m$ - параметр, равный общему значению нескольких совпадающих друг с другом линейных сложностей $r_{i}$ и, как правило, введенный в соответствующем предложении в тексте статьи. Например, для предложения 11 параметр $m$ - это общее значение $r_{1}=r_{2}=r_{3}=r_{10}=r_{13}=r_{15}$.

В таблице примеров цифра в каждом столбце $i=1, \ldots, 18$ означает значение $r_{i}$ соответствующей линейной сложности. Так, в примере $1 r_{1}=r_{2}=r_{3}=1, r_{4}=\infty$, $r_{5}=1$, и т.д. Прочерк означает, что данная линейная сложность не определена, пробел - что она не найдена. Большинство (но не все) из значений линейной сложности в таблице примеров были вычислены в тексте работы. Отметим, что во всех примерах параметр $\nu=1$, за исключением примеров 5 и 10, в которых $\nu=2$.

Автор признателен В. П. Елизарову за обсуждение ряда результатов статьи.

\section{Список литературы}

1. Атья М., Макдональд И., Введение в хоммутативную алгебру. Мир, Москва, 1972.

2. Ван дер Варден Б. Л., Алгебра. Наука, Москва, 1979.

3. Елизаров В. П., Конечнъе колъца. М., 1993.

4. Каш Ф. Модули и кольца. Мир, Москва, 1981.

5. Кон П., Свободнъе колъца и их свлзи. Мир, Москва, 1975.

6. Кузьмин А. С., Куракин В. Л., Нечаев А. А., Псевдослучайные и полилинейные последовательности. Труды по дискретной математике, том 1. Научное изд-во ТВП, 1997, 139-202.

7. Куракин В. Л., Биномиальное представление линейных рекуррентных последовательностей. Фундаментальная и прихладная математиха (1995) 1, №2, 553-556.

8. Куракин В. Л., Алгоритм Берлекэмпа-Месси над конечными кольцами, модулями и бимодулями. Дискретная математика (1998) 10, №4, 3-34.

9. Куракин В. Л., Полиномиальные преобразования линейных рекуррентных последовательностей над конечными коммутативными кольцами. Дискретная математика (2000) 12, №3, 3-36.

10. Нечаев А. А., Линейные рекуррентные последовательности над квазифробениусовыми модулями. Успехи матем. наух, (1993) 48, N3, 197-198.

11. Нечаев А. А., Конечные квазифробениусовы модули, приложения к кодам и линейным рекуррентам. Фундаментальная и прикладная математика (1995) 1, №1, 229-254.

12. Херстейн И., Нехоммутативные колъца. Мир, Москва, 1972.

13. Шафаревич И. Р., Основные понятия алгебры. Современные проблемы математихи. Фундаменталънье направления. Итоги науки и техники, т. 11. ВИНИТИ, Москва, 1986.

14. Herlestam T., On the complexity of functions of linear shift register sequences. Int. Symp. Inform. Theory, Les Arc, France, 1982. 
15. Key E. L., An analysis of the structure and complexity of nonlinear binary sequence generators. IEEE Trans. Inform. Theory (1976) 22, №6, 732-736.

16. Kurakin V. L., Kuzmin A. S., Mikhalev A. V., Nechaev A. A., Linear recurrences over rings and modules. J. Math. Sci. (1995) 76, №6, 2793-2915.

17. V. L. Kurakin, A. V. Mikhalev, A. A. Nechaev, V. N. Tsypyschev, Linear and polylinear recurring sequences over abelian groups and modules. J. Math. Sci. (2000) 102, №6, 45984626.

18. McCoy N. H., Rings and ideals. Math. Assoc. Amer., Menasha, 1962.

19. Peterson B., Taft E. Y., The Hopf algebra of lineary recursive sequences. Aequat. Math. (1980) 20, 1-17.

20. Rueppel R. A., Staffelbach O. J., Products of linear recurring sequences with maximum complexity. IEEE Trans. Inform. Theory (1987) 33, №1, 126-131.

21. Sakata S.,. Synthesis of two-dimensional linear feedback shift-registers and Groebner bases. Lect. Notes Comput. Sci. (1989) 356, 394-407.

22. Sakata S., Extension of the Berlekamp-Massey algorithm to $N$ dimensions. Inform. and Comput. (1990) 84, №2, 207-239.

23. Sakata S., Two-dimensional shift register synthesis and Groebner bases for polynomial ideals over an integer residue ring. Discr. Appl. Math. (1991) 33, №1-3, 191-203.

24. Zierler N., Mills W. H., Products of linear recurring sequences. J. Algebra (1973) 27, №1, 147-157.

Статья поступила 14.12.2000. 